\title{
TASK-BAsed Lens Design, With Application to Digital Mammography
}

\author{
by \\ Liying Chen
}

$\overline{\text { Copyright (C) Liying Chen } 2004}$

A Dissertation Submitted to the Faculty of the

Committee on Optical Sciences (Graduate)

In Partial Fulfillment of the Requirements

For the Degree of

DOCTOR OF PHILOSOPHY

In the Graduate College

The University of ARIzona

2004 
UMI Number: 3145053

Copyright 2004 by

Chen, Liying

All rights reserved.

\section{INFORMATION TO USERS}

The quality of this reproduction is dependent upon the quality of the copy submitted. Broken or indistinct print, colored or poor quality illustrations and photographs, print bleed-through, substandard margins, and improper alignment can adversely affect reproduction.

In the unlikely event that the author did not send a complete manuscript and there are missing pages, these will be noted. Also, if unauthorized copyright material had to be removed, a note will indicate the deletion.

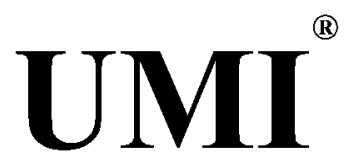

UMI Microform 3145053

Copyright 2004 by ProQuest Information and Learning Company.

All rights reserved. This microform edition is protected against unauthorized copying under Title 17, United States Code.

ProQuest Information and Learning Company 300 North Zeeb Road

P.O. Box 1346

Ann Arbor, MI 48106-1346 
The University of Arizona ${ }^{\circledR}$

Graduate College

As members of the Final Examination Committee, we certify that we have read the dissertation prepared by LiYing Chen

entitled Task-Based Lens Design With Applications To Digital Mammography

and recommend that it be accepted as fulfilling the dissertation requirement for the Degree of Doctor of Philosophy

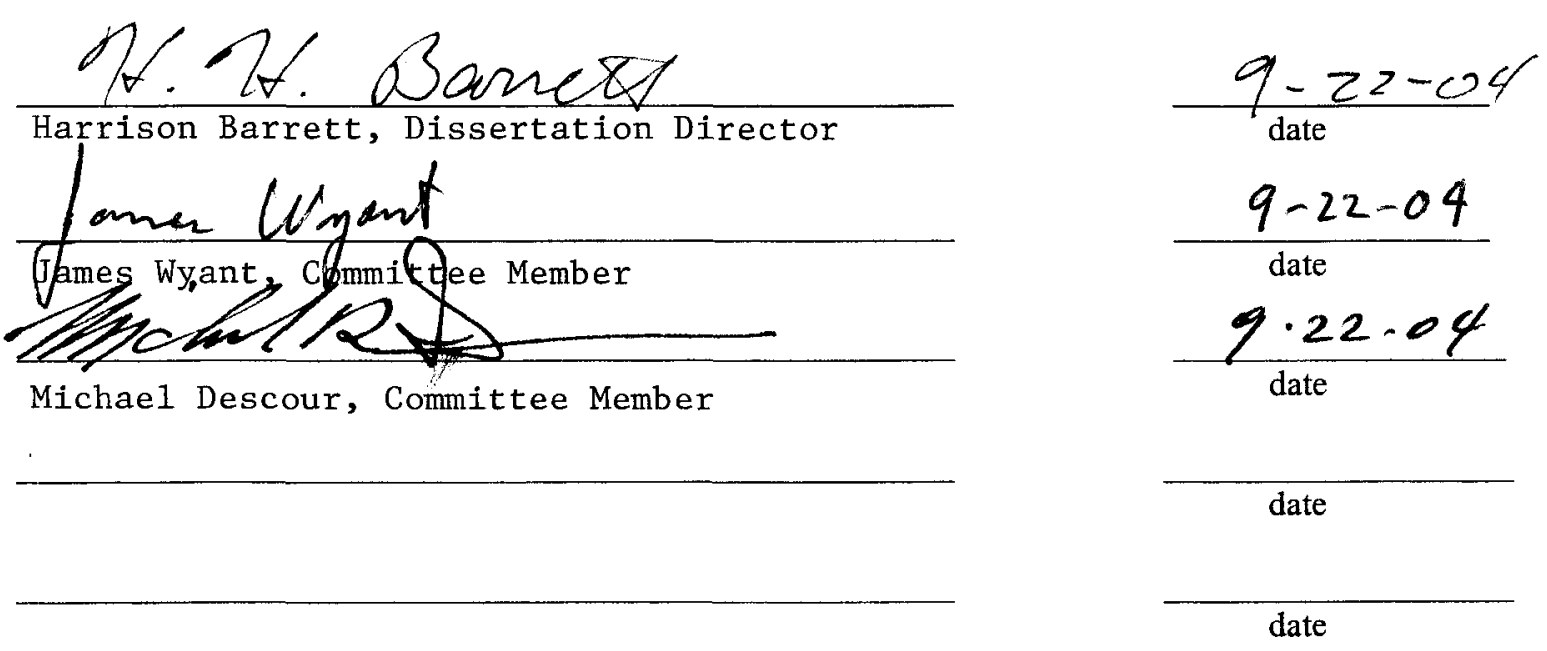

Final approval and acceptance of this dissertation is contingent upon the candidate's submission of the final copies of the dissertation to the Graduate College.

I hereby certify that I have read this dissertation prepared under my direction and recommend that it be accepted as fulfilling the dissertation requirement.

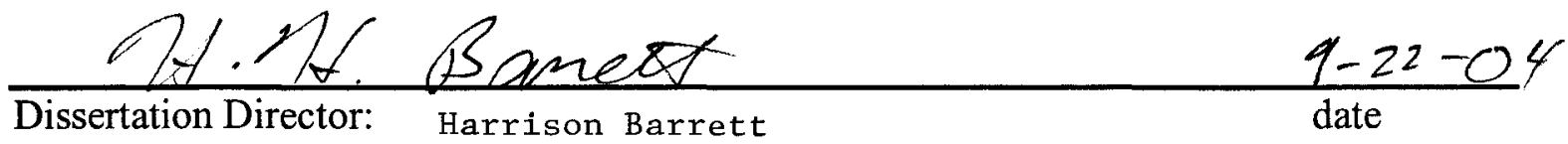




\section{Statement By AUthor}

This dissertation has been submitted in partial fulfillment of requirements for an advanced degree at The University of Arizona and is deposited in the University Library to be made available to borrowers under rules of the Library.

Brief quotations from this dissertation are allowable without special permission, provided that accurate acknowledgment of source is made. Requests for permission for extended quotation from or reproduction of this manuscript in whole or in part may be granted by the copyright holder.

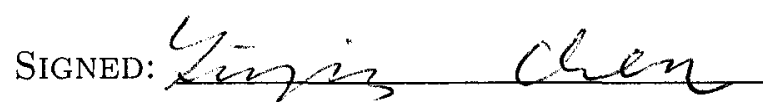




\section{ACKNOWLEDGMENTS}

I'll be deeply grateful to my advisor, Dr. Harrison H. Barrett. This dissertation would not have been possible without the efforts of Harry Barrett. He has been an invaluable source of wisdom and guidance throughout my graduation education. As an advisor, he has been mentoring me for all these years as a excellent model in doing research creatively and finding solutions precisely.

I would like to thank all the other members of my dissertation committee, Dr. Jim Wyant and Dr. Michael Descour. I gratefully acknowledge the active researchers in the medical imaging field who have helped me, Dr. Kyle Myers, Dr. Eric Clarkson, Dr. Matthew Kupinski and Dr. Janick Rolland. They have given me insightful input on this work and general comments on the relative topics in medical imaging.

The friendly people in and around the Radiology research building have made it a pleasure for me to work here.I appreciate the opportunity to work in such a great team of students, teachers, and scientists.

Finally, I would like to thank my parents, Wenjian Chen and Shujing Yu, and my husband Yong Xie for their endless love and support in all my educational endeavors. 


\section{TABle of Contents}

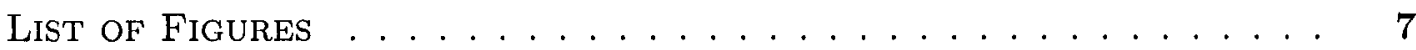

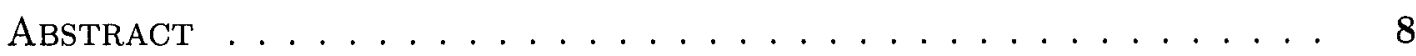

Chapter 1. Introduction $\ldots \ldots \ldots \ldots \ldots \ldots$

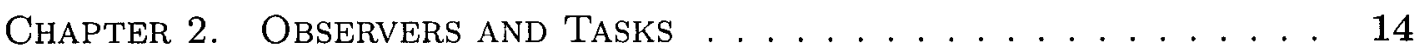

2.1. Classification Tasks . . . . . . . . . . . . . . . . . 15

2.1.1. Binary detection tasks ....................... 15

2.1.2. Multiple-hypothesis classification ............ 22

2.2. Estimation Task . . . . . . . . . . . . . . . . . . . . . . 25

2.2.1. Bayesian Observers . . . . . . . . . . . . . . . . . 25

2.2.2. Performance Measures . . . . . . . . . . . . . 27

Chapter 3. System Description and Modeling . . . . . . . . . . 29

3.1. Physical System . . . . . . . . . . . . . . . . . . . . . . . . . . . . 32

3.2. Mathematical Model . . . . . . . . . . . . . . . . . 35

3.2.1. Point process of absorbed x-ray photons in the screen . . . . 35

3.2.2. Point process of incident visible photons on the lens . . . . . 37

3.2.3. Point process of output visible photons from the lens . . . . . 43

3.2.4. Point process of visible photons in the image . . . . . . . . . 44

3.2.5. Random image vector ... . . . . . . . . . 46

Chapter 4. Merit Function - SNR . . . . . . . . . . . . 47

4.1. Tasks and Observers Model . . . . . . . . . . . . . . . . . . . 47

4.2. Procedures . . . . . . . . . . . . . . . . . . . . . . . . . . . . . . . . . . 48

4.3. Formulas . . . . . . . . . . . . . . . . . . . . 49

Chapter 5. Characteristic Functional Analysis . . . . . . . 53

5.1. Characteristic Functionals ................. . . 54

5.2. Transformation Through Imaging Systems . . . . . . . . . . . . 54

5.3. Object Models . . . . . . . . . . . . . . . . . . . . . . 57

5.4. Characteristic Functionals Through the Model Imaging System . . . . 62

5.4.1. CFl of absorbed x-ray photon processes . . . . . . . . . . 62

5.4.2. $\mathrm{CFl}$ of incident visible photon processes on the lens . . . . . 66

5.4.3. CFl of visible photon processes in images . . . . . . . . . . 70

5.4.4. CF of image vectors ..................... 75

5.4.5. Results for lumpy backgrounds . . . . . . . . . . . 76 
TABle of Contents-Continued

5.5. FOM Derivation . . . . . . . . . . . . . . . . . 76

5.5.1. Fréchet derivative . . . . . . . . . . . . . 76

5.5.2. Calculations of clustered lumpy backgrounds . . . . . . . 79

Chapter 6. Numerical Simulations . . . . . . . . . . . . . 84

6.1. Backgrounds and Signals . . . . . . . . . . . . . . . . . 86

6.2. Scattering Model of Screens . . . . . . . . . . . . . . . . . 88

6.3. Optical Simulation . . . . . . . . . . . . . . . . . . . . . . 92

6.4. Positive Semi-definite Functions . . . . . . . . . . . . . . . . . 93

6.5. Channel Profiles . . . . . . . . . . . . . . . . . . . 94

Chapter 7. Results ........................ 98

7.1. Aperture Size . . . . . . . . . . . . . . . . . . 100

7.2. Working Distance . . . . . . . . . . . . . . . . . 102

7.3. Defocus . . . . . . . . . . . . . . . . . . 104

Chapter 8. Conclusions and Future Work . . . . . . . . . . . 112

8.1. Conclusions . . . . . . . . . . . . . . . . . . . 112

8.2. Future Work . . . . . . . . . . . . . . . . . . . 112

Appendix A. Mean Image Vector . . . . . . . . . . . . . . . 115

Appendix B. Covariance Matrix . . . . . . . . . . . . . . . . 118

Appendix C. Covariance Function Decomposition . . . . . . . . 121

Appendix D. Probability-Generating Function . . . . . . . . . 126

REFERENCES . . . . . . . . . . . . . . . . . . . . . 128 


\section{List OF FIGURES}

Figure 3.1. The layout of the model system . . . . . . . . . . . . . . . 31

Figure 6.1. Two samples of the clustered lumpy background . . . . . . . . 97

FigURE 7.1. SNR vs. aperture . . . . . . . . . . . . . . . . 106

Figure 7.2. Spot diagrams at different aperture . . . . . . . . . . . 107

FIGURE 7.3. SNR vs. working distance . . . . . . . . . . . . . . 108

FigURE 7.4. Spot diagrams at different working distances . . . . . . . . . . 109

Figure 7.5. SNR vs. defocus . . . . . . . . . . . . . . . . . . . . 110

Figure 7.6. Spot diagrams at different defocus . . . . . . . . . . . . . . 111 


\begin{abstract}
Recent advances in model observers that predict human perceptual performance now make it possible to optimize medical imaging systems for human task performance. We illustrate the procedure by considering the design of a lens for use in an optically coupled digital mammography system. The channelized Hotelling observer is used to model human performance, and the channels chosen are differences of Gaussians (DOGs). The task performed by the model observer is detection of a lesion at a random but known location in a clustered lumpy background mimicking breast tissue. The entire system is simulated with a Monte Carlo application according to the physics principles, and the main system component under study is the imaging lens that couples a fluorescent screen to a CCD detector. The SNR of the channelized Hotelling observer is used to quantify the detectability of the simulated lesion (signal) upon the simulated mammographic background. In this work, plots of channelized Hotelling SNR vs. signal location for various lens apertures, various working distances, and various focusing places are shown. These plots thus illustrate the trade-off between coupling efficiency and blur in a task-based manner. In this way, the channelized Hotelling SNR is used as a merit function for lens design.
\end{abstract}




\section{CHAPter 1}

\section{INTRODUCTION}

The systematic method of lens design in use today is an iterative technique. By this procedure, a basic lens type is first picked. A paraxial thin lens and then a thick lens solution are next developed. Then, with the great progress in computers and applied mathematics today, one can directly go to optimization by relatively high-speed ray trace. Any automatic computer optimization program actually drives an optical design to a local minimum, as defined by a merit function. The name "merit function" is a misnomer since it is in fact a de-merit function. Broadly speaking, the merit function can be described as a function of calculated characteristics, which is intended to completely describe the value or the quality of a given lens design. The typical merit function is the weighted sum of the squares of many image defects, among which are various aberration coefficients, spot sizes, RMS wavefront error (WFE) and/or MTF at one or more locations in the field of view. Some merit functions consider the boundary conditions for the lens as well. These boundary conditions include such items as maintaining the effective focal length, center and edge spacings, overall length, etc. Choice of terms to be included in the merit function is a critical part of the design process. The basis for selecting the aberrations is the experience of designers and their understanding of the nature of lenses and aberrations. The exact choice of term weights and balancing aberrations is largely an empirical process. A large amount of effort has been expended to improve the optimization procedure, while the choice of merit function to provide the best image quality is still not so clear.

Although the aberration coefficients give designers an idea of the performance of the system, the overall effect of these aberrations on the final image is hard to quantify. The question is: What effect does a given amount of aberration have on 
the performance of optical imaging systems? Phrased in this way, the question is rather vague, and more concrete definitions of image quality are needed. A scientific or medical image is always produced for some specific purpose or task, and the only meaningful measure of its quality is the appropriate observer's performance on that task. The basic difficulty with the weighted sums of the squared errors as measures of image quality is that they do not take into account the observer and the task of images. A task-based approach to assessment of image quality [5, 8, 6, 33, 39, 52, 69] must therefore start with a specification of the task, and then determine quantitatively how well the observer performs the task. Generally, the tasks of practical importance can be either to classify the image into one of two or more categories or to estimate one or more quantitative parameters of the object from the image data. We call these two kinds of tasks classification and estimation, respectively. In astronomical imaging, for example, one might want to detect the presence of a small companion star near a brighter star, or one might want to estimate the relative magnitude of the two stars. The first problem is a classification task, while the second is an estimation task. The observer can be either a human or some mathematical model observer such as a machine classifier or pattern-recognition system. With the figure of merit for each task, we can quantitatively measure the task-based image quality of imaging systems.

One challenging lens design area is medical x-ray imaging systems, which are usually designed to produce images having the highest quality possible for diagnosis within a specified dose. One type of digital x-ray imaging systems uses optics to couple $\mathrm{x}$-ray fluorescent screens onto detection devices or image receptors such as CCD arrays. The lens design must meet a very stringent requirement because the radiation dose applied to patients is limited. Within the safe dose level, x-ray imaging systems should be quantum-limited, which means that the dominant noise is the quantum noise from the fluctuation in the x-ray photons. Though $\mathrm{x}$-ray phosphor screens may create hundreds of visible or UV photons per x-ray photon absorbed, the 
imaging optics cannot couple all of them onto an image receptor. In fact, the number of transmitted light quanta is so much less than the number of those created that the imaging optics can easily become the quantum sink in the whole $\mathrm{x}$-ray imaging system $[12,18,45,49,54]$. In its simplest interpretation, when we represent an imaging system as a series of cascaded processes or stages, the stage with the fewest quanta per incident $\mathrm{x}$-ray photon is called the quantum sink. The cascaded model is one form of analyses often used by system designers. In this model, the quanta leaving one stage contribute an effective input to the subsequent stage. The starting process usually results from the Poisson statistics characterizing the incident x-ray photons. The randomness introduced by Poisson statistics is a basic noise in photon-limited imaging systems, but there always exists additional noise from external sources, such as electronic components. In designing or assessing imaging systems, it is important to understand the processes that contribute to the image formation and the effects they have on image signal and noise. In the cascaded model, the noise propagates through all stages to the final image as well. All sorts of blurring, especially from aberrations, introduce changes in the appearance and amplitude of the statistical xray fluctuations. Such noise takes on a characteristic mottled appearance. Only fast lenses can couple enough light onto image receptors to make recognizable contrast and satisfactory signal intensity over noise. At the same time, the inherent severe aberrations of fast lenses can make the mottled appearance even worse.

Recent advances in model observers that predict human perceptual performance now make it possible to optimize optical imaging systems, such as lenses, for human task performance. We illustrate the evaluation procedure of lens quality by investigating a design of a lens for use in an optically coupled digital mammographic imaging system. The cascaded imaging chain consists of a fluorescent screen, an imaging lens, and an electronic imaging device such as a CCD camera. Observers' performance is evaluated based on the statistical properties of the images from the system. The statistics of images is determined by the statistics of objects and of the 
noise from imaging systems. The optimization will be built on this evaluation step. The task-based lens design is then practical with the optimization procedure.

An introduction to the general theory on tasks and observers is in chapter 2, and the basics of the statistical decision theory are presented oriented toward taskbased image quality evaluation methods. The observer's performance is quantified by several figures of merits, one of which, observer signal-to-noise ratio (SNR), is going to be the merit function in the lens optimization. The formulas are the basis of the merit function definition afterward.

A detailed model of the system to be optimized is described in chapter 3 . In particular, an optically coupled digital $\mathrm{x}$-ray mammographic imaging system is chosen to apply the general image quality evaluation discussed before. At first, the physical system is discussed carefully including the important assumptions useful in establishing the mathematical model later. The mathematical model is then described rigorously in steps. Stochastic point processes are the central mathematical tool in the model so that the statistics of the intermediate output at each stage of the imaging chain can be defined completely. After going through the mathematical system model, we can obtain the statistics of the output image at the final stage. The image statistics are the fundamental requirement for the observer SNR, which is an objective merit function in lens design.

The objective merit function in lens design is analyzed in chapter 4. After observing the statistical properties of each stage in the mathematical system model, we summarize the procedure to calculate the objective merit function, or the observer SNR, for designing the lens. Following this procedure, we derive a concise expression of the observer SNR, which is useful in numerical computation.

In chapter 5, we introduce a new mathematical concept, characteristic functionals of stochastic processes, and discuss use of the functionals in system analysis. There is a formal way to derive all moments of a stochastic process from its characteristic functional by repeated derivatives. Since the observer SNR only needs the first two 
moments, it is always possible to derive SNR from the characteristic functional of the image process. Moreover, characteristic functionals give the full knowledge of stochastic processes, equivalent to probability density functions (PDF). It can facilitate other applications requiring higher-order moments, like ideal observers and object statistics estimation.

In chapter 6 , we discuss the Monte Carlo strategy used in numerically evaluating the objective merit function in lens design. The types of signals and backgrounds are chosen specifically for mammography. The model of the scattering of visible light inside fluorescent screens is discussed. Also, some numerical computing strategies are implemented to avoid the unacceptable errors.

Some case studies are in chapter 7. Specifically, three types of parameters in lens design are chosen to investigate their effects on the objective image quality.

The conclusions and suggestions for future work are in chapter 8. 


\section{Chapter 2}

\section{OBSERVERS AND TASKS}

Image quality is a statistical concept with statistical decision theory as the key. It is defined as how well a decision can be made using an image as input. Image quality must be assessed as the average performance of some observer on some specific decision task.

There are generically two types of tasks: classification and estimation. Both kinds of problems have similar basic structure including four components $[48,67]$. The first component is the message space. The second is the statistical transition mechanism, and the third is the observation space. The message space is probabilistically mapped onto the observation space by the means of the transition mechanism. The last component is the decision rule. In classification tasks, the message space has a finite number of points. Each point is also called a hypothesis. The decision rule is to guess which hypothesis is true after observing the outcome in the observation space. If the number of points in the message space is uncountably infinite, the task is referred to as an estimation task. The decision rule, or the estimation rule now, is the mapping of the observation space into an estimate. The message space in estimation tasks is also called the parameter space, and the parameter is what we want to estimate. The decision rule is the observer who performs the task. The observer might be a human or a mathematical algorithm which is known as a model observer. Model observers make it possible to optimize imaging systems without the need for costly human-observer studies.

To evaluate image quality, we need some way to tell how well the observer performs. The figures of merit are defined differently for each kind of tasks. Useful figures of merit for classification tasks include the area under the receiver operating 
characteristic (ROC) curve (AUC) and the classification signal-to-noise ratio (SNR). The figures of merit for estimation tasks include Bayes risk, mean-square error (MSE) and ensemble mean-square error (EMSE).

When evaluating medical images, we will encounter both kinds of tasks. If a doctor needs to diagnose a disease based on an image, he or she performs a classification task, and the two underlying hypotheses are disease-present or normal. When measuring the size of a cancer tissue, a doctor is performing a estimation task. The message space is not the same as the object space in imaging system analysis. The objects viewed by imaging systems can be, for example, the x-ray absorption distribution inside a patient in $\mathrm{x}$-ray imaging. An imaging system together with a noise mechanism is the transition mechanism mapping object space to data space, and each point in data space is an output image. The data space is the observation space. We will use these two terms interchangeably from now on. For the same task, different imaging systems may look at different objects and produce different images. The performance of the same observer can vary with imaging systems. The better the performance, the higher the pertinent figure of merit, and the better quality is the imaging system. Medical imaging systems should therefore be optimized according to the tasks performed on the output images. The corresponding figure of merit is the measure of the quality of imaging systems.

The detailed discussion on both tasks is in the next sections. Classification tasks are the first to be considered. The development of estimation-task treatment follows next because it is analogous to classification problem.

\subsection{Classification Tasks}

\subsubsection{Binary detection tasks}

At first, we will consider the simplest type of classification problems: two-hypothesis classification tasks, also known as binary detection tasks. Binary detection problems 
illustrate most of the concepts in the whole statistical decision theory. In these problems, an observer needs to make decisions between two hypotheses, $H_{0}$ and $H_{1}$. When its test statistic is larger than the threshold, the observer makes decision $D_{1}$; otherwise decision $D_{0}$ is made. The decision rule is a strategy to map the observation space into the binary message space. It is equivalent to dividing the data space into two regions such that the image in each region will correspond to one of the decisions. The regions must be disjoint to avoid ambiguous decisions, and they must cover the whole data space to ensure each image is associated with a decision.

Bayesian observer One of the decision criteria is the Bayes criterion. It assumes that the observer has all the statistical information regarding the task, including the conditional probability densities $\operatorname{pr}_{\mathbf{g}}\left(\mathbf{g} \mid H_{0}\right), \operatorname{pr}_{\mathbf{g}}\left(\mathbf{g} \mid H_{1}\right)$, and the prior probabilities $\operatorname{Pr}\left(H_{0}\right)$ and $\operatorname{Pr}\left(H_{1}\right)$. A cost is assigned to each possible decision and truth pair. The Bayesian observer uses the knowledge to minimize the average cost over both the data and the truth. The average cost is called the Bayes risk. We denote the cost for the four possible pairs as $C_{00}, C_{10}, C_{01}$ and $C_{11}$. The first subscript indicates the decision and the second, the hypothesis that is true. The Bayes risk is therefore

$$
\bar{C}=\sum_{i=0}^{1} \sum_{j=0}^{1} C_{i j} \operatorname{Pr}\left(D_{i} \mid H_{j}\right) \operatorname{Pr}\left(H_{j}\right)
$$

We can write the conditional probabilities $\operatorname{Pr}\left(D_{i} \mid H_{j}\right)$ in terms of the conditional probability densities $\operatorname{pr}_{\mathbf{g}}\left(\mathbf{g} \mid H_{j}\right)$,

$$
\operatorname{Pr}\left(D_{i} \mid H_{j}\right)=\int_{Z_{i}} \mathrm{~d} \mathbf{g} \operatorname{pr}_{\mathbf{g}}\left(\mathrm{g} \mid H_{j}\right),
$$

where $Z_{i}$ is the region corresponding to the decision $D_{i}$ in the data space. We also assume that the cost of a wrong decision is higher than the cost of a correct decision. In other words,

$$
C_{10}>C_{00}, \quad C_{01}>C_{11}
$$


When choosing the decision regions $Z_{0}$ and $Z_{1}$ to minimize the Bayes risk, we find the final decision strategy is [67]

$$
\frac{\operatorname{pr}_{\mathbf{g}}\left(\mathbf{g} \mid H_{1}\right)}{\operatorname{pr}_{\mathbf{g}}\left(\mathbf{g} \mid H_{0}\right)} \underset{D_{0}}{\stackrel{D_{1}}{\gtrless}} \frac{\operatorname{Pr}\left(H_{0}\right)\left(C_{10}-C_{00}\right)}{\operatorname{Pr}\left(H_{1}\right)\left(C_{01}-C_{11}\right)} .
$$

The expression on the left is called the likelihood ratio and denoted by $\Lambda(\mathrm{g})$. The likelihood ratio is the scalar test statistic for the Bayesian observer, and the expression on the right side is the threshold. We can see that all the data processing is in computing of $\Lambda(\mathrm{g})$ and independent of the priors or costs. In practice, the prior probabilities or the costs are seldom known. The strategy enables us to build the data processor and leave the threshold as a variable to accommodate changes.

ROC curve Mathematical observers compute a scalar test statistic $t(\mathrm{~g})$ which is the outcome of some operation on the data $\mathrm{g}$. Observers compare $t$ with a threshold $t_{c}$, and make decision $D_{1}$ if $t$ is higher or $D_{0}$ otherwise. There are four possible decision and underlying truth pairs in binary detection problems [9]:

1. true positive(TP): $\quad H_{1}$ is true and $D_{1}$ is made.

2. false positive(FP): $\quad H_{0}$ is true and $D_{1}$ is made.

3. false negative(FN): $\quad H_{1}$ is true and $D_{0}$ is made.

4. true negative(TN): $\quad H_{0}$ is true and $D_{0}$ is made.

The conditional probabilities $\operatorname{Pr}\left(D_{i} \mid H_{j}\right)$ are also called the fraction of the corresponding scenarios. In fact, the value of true-positive fraction (TPF) $\operatorname{Pr}\left(D_{1} \mid H_{1}\right)$ and false-positive fraction $(\mathrm{FPF}) \operatorname{Pr}\left(D_{1} \mid H_{0}\right)$ can specify all the possible outcomes:

$$
\begin{aligned}
& \mathrm{TPF}=\operatorname{Pr}\left(t \geq t_{c} \mid H_{1}\right)=\int_{t_{c}}^{\infty} \mathrm{d} t \operatorname{pr}\left(t \mid H_{1}\right)=1-\mathrm{FNF}, \\
& \mathrm{FPF}=\operatorname{Pr}\left(t \geq t_{c} \mid H_{0}\right)=\int_{t_{c}}^{\infty} \mathrm{d} t \operatorname{pr}\left(t \mid H_{0}\right)=1-\mathrm{TNF} .
\end{aligned}
$$

Because $t$ is a function of the random variable $\mathbf{g}$, the test statistic is random itself. 
We must evaluate the performance of the observers. The TPF and FPF are both functions of the threshold $t_{c}$. We can generate a plot of the TPF versus FPF with $t_{c}$ as the varying parameter. This plot is referred to as the receiver operating characteristic (ROC) curve. It completely describes the performance of the observer. In particular, it is possible to show [67] that all ROC's have the following properties:

1. All continuous likelihood-ratio tests have ROC's that are concave downward.

2. All continuous likelihood-ratio tests have ROC's that are above the TPF $=\mathrm{FPF}$ line.

3. The slope of a curve in a ROC at a particular point is equal to the value of the threshold required to achieve the TPF and FPF of that point.

The area under the ROC curve (AUC) is a figure of merit for classification tasks [9]. It is defined as

$$
\mathrm{AUC}=\int_{0}^{1} \mathrm{dFPF} \mathrm{TPF}(\mathrm{FPF}) .
$$

Because both the TPF and the FPF range from 0 to 1 , the AUC also range from 0 to 1 .

Whenever the maximum value of the Bayes risk is interior to the interval $(0,1)$ on the prevalence $\operatorname{Pr}\left(H_{1}\right)$, the maximum true-positive fraction at a specific false-positive fraction is on the ideal observer's ROC curve [67]. Therefore, the Bayesian observer's ROC curve is the upper bound of all the other observers. The AUC is then maximized by the Bayesian observer, and the performance of the Bayesian observer provides an upper bound of all the other observers performance.

Hotelling observer Although the ideal observer is the optimal observer among all observers, the ideal observer's test statistic $\Lambda(\mathrm{g})$ is often nonlinear on the data $\mathbf{g}$ and difficult to compute. The linear observers have the test statistics linear on the data,

$$
t(\mathrm{~g})=\mathbf{w}^{t} \mathbf{g}
$$


where $\mathbf{w}$ is the observer's template vector with the same dimension as image vectors, and $\mathbf{w}^{t} \mathbf{g}$ denotes the inner product of the template and the image. Among them, the optimal linear observer is the Hotelling observer [36]. It has the template as follows

$$
\begin{aligned}
\mathbf{w}_{H o t} & =\mathbf{K}_{\mathbf{g}}^{-1} \Delta \overline{\mathbf{g}}, \\
\mathbf{K}_{\mathbf{g}} & =\frac{1}{2}\left(\mathbf{K}_{1}+\mathbf{K}_{0}\right), \\
\Delta \overline{\mathbf{g}} & =\overline{\mathbf{g}}_{1}-\overline{\mathbf{g}}_{0},
\end{aligned}
$$

where $\mathbf{K}_{1}, \mathbf{K}_{0}, \overline{\mathbf{g}}_{1}$ and $\overline{\mathbf{g}}_{0}$ are the covariance matrices and the mean image vector under each hypothesis, respectively.

The Hotelling observer is optimal among linear observers in the sense of another figure of merit for image quality in binary detection tasks. This measure of image quality is called the observer signal-to-noise ratio (SNR), and will be discussed below. We should notice that the Hotelling observer may not maximize the AUC among all the linear observers. The AUC-optimal linear discriminants are discussed in Ref. [9].

The ideal observer is identical to the Hotelling observer when the data are normally distributed with the same covariance matrix under both hypotheses. In essence, the Hotelling observer treats the data as normally distributed regardless of the real statistics of the data. Therefore, the Hotelling observer has the advantage of requiring only the first two moments of the data.

SNR The AUC is a measure of the class separability in binary detection tasks. Another measure of separability is the SNR defined as

$$
\mathrm{SNR}=\frac{\langle t\rangle_{1}-\langle t\rangle_{0}}{\sqrt{\frac{1}{2} \sigma_{0}^{2}+\frac{1}{2} \sigma_{1}^{2}}}
$$

where $t$ is the test statistic, and $\sigma_{0}$ and $\sigma_{1}$ are the variances of $t$ under both hypotheses respectively. The SNR of the Hotelling observer can be found as

$$
\mathrm{SNR}_{H o t}^{2}=\Delta \overline{\mathrm{g}}^{t} \mathbf{K}_{\mathrm{g}}^{-1} \Delta \overline{\mathrm{g}}
$$


The test statistic of the ideal observer is the likelihood ratio $\Lambda$ or any monotonic transform of it such as the log-likelihood ratio $\lambda$. The SNR of the ideal observer is thus defined as the basic definition with the test statistic $t$ being $\Lambda$ or some monotonic function of it. When $\lambda$ is used in the definition, the SNR of the ideal observer can be simplified and take on the same form as that of the Hotelling observer only in the signal-known-exactly and background-known-exactly (SKE/BKE) cases with Gaussian noise.

When the test statistic is Gaussian distributed under both hypotheses, AUC can be monotonically mapped to SNR. The mapping is through the error function,

$$
\mathrm{SNR}_{H o t}=2 \operatorname{erf}^{-1}(2 \mathrm{AUC}-1)
$$

For non-Gaussian test statistics, the result of this mapping is called the detectability index $d_{A}$. This figure of merit should be used with caution if the test statistic is not normally distributed. In particular, when the test statistic is skewly distributed, the variance can not correctly measure the spread of the test statistic, so that the SNR can not indicate the separability well.

Human observer and channelized Hotelling observer Many imaging modalities have a human as the end user of the images. The task is almost always classification because humans are not as good as a computer algorithm at parameter calculation. Human observer's performance under this task can be measured from psychophysical studies, such as two-althernative-forced-choice (2AFC) experiments. For human observers in 2AFC experiments, a decision has to be made between two presented images, one generated under hypothesis $H_{0}$ and the other under $H_{1}$. The correct proportion has the same value as the AUC of human.

Psychophysical experiments on human observer are usually limited by resources and time. Some mathematical observers modeling the human observer can be very helpful when investigating imaging systems for human users. With the vast amount of 
research results from the studies on all aspects of human visual system, many models have been developed for different purpose. We will focus on the model developed specifically for the task-based evaluation of imaging systems for classification tasks.

The notion of channels in the human visual system has been studied intensively in vision science for many years [31]. The application of channelized observers to medical image-quality assessment began with the work of Myers and Barrett [51], who introduced the channelized Hotelling observer. Based on previous work in our group [2], the Hotelling observer with a set of channels can satisfactorily predict human observer's performance in a wide range of situations when the tumor profile is deterministic. The choice of the set of channel profiles should be based on the human visual system study, and we will briefly discuss the human visual system and the channel set we are using in a later chapter.

The channelized Hotelling observer applies Hotelling observer's strategy on the channel output of the image. The channel output is defined by the transformation

$$
\mathbf{u}=\mathbf{T}^{t} \mathbf{g}
$$

where each column of the matrix $\mathbf{T}$ represents the spatial profile of a channel. The channelized Hotelling observer applies the linear strategy on the channel output as follows

$$
t=\mathrm{w}_{c h}^{t} \mathbf{u}
$$

where $\mathbf{w}_{c h}$ is the template of the channelized Hotelling observer. The template is given by

$$
\mathbf{w}_{c h}=\mathbf{T}\left(\mathbf{T}^{t} \mathbf{K}_{\mathbf{g}} \mathbf{T}\right)^{-1} \mathbf{T}^{t} \Delta \overline{\mathbf{g}}
$$

where $\mathbf{K}_{\mathbf{g}}$ and $\Delta \overline{\mathrm{g}}$ are defined in (2.1.10) and (2.1.11). The performance of the channelized Hotelling observer can be measured by the $\mathrm{SNR}_{c h}$ as follows

$$
\mathrm{SNR}_{c h}^{2}=\Delta \overline{\mathbf{g}}^{t} \mathbf{T}\left(\mathbf{T}^{t} \mathbf{K}_{\mathrm{g}} \mathbf{T}\right)^{-1} \mathbf{T} \Delta \overline{\mathrm{g}}
$$


The AUC of the channelized Hotelling observer is difficult to get directly unless the probability density functions or the characteristic functions of images under both hypotheses have analytical forms and can be integrated numerically over the hyperplane normal to the template [9]. By the central limit theorem, the test statistic $\mathbf{w}_{c h}^{t} \mathbf{u}$ is approximately normally distributed since it is a linear combination of all the image vector elements. The SNR can thus be transformed into the AUC of the observer by the inverse of the monotonic transform in (2.1.14). The human observer's performance can be compared with that of the channelized Hotelling observer by comparing AUC of each observer. We will use the SNR since a monotonic transformation preserves the order of different systems' performance.

\subsubsection{Multiple-hypothesis classification}

Now we will consider the classification tasks with multiple hypotheses, choosing one among $L$ hypotheses. In a binary problem, a scalar test statistic is used to separate the data space into two regions. In a $L$-class problem, we will need more than one scalar functions on the data to divide the data space into $L$ regions without ambiguity. Each region corresponds to a class or hypothesis. The test statistic is a multi-dimensional vector function.

The Bayesian observer selects the decision regions $Z_{j}, j=1, \ldots, L$, such that the Bayes risk $\bar{C}$ is minimized. The cost for the $L$-class problem is a group of cost values $\left\{C_{i j}: i, j=1 \ldots L\right\} . C_{i j}$ is the cost associated with making decision $D_{i}$ in favor of hypothesis $H_{i}$ when hypothesis $H_{j}$ is true. The Bayes risk is therefore

$$
\bar{C}=\sum_{i=1}^{L} \sum_{j=1}^{L} C_{i j} \operatorname{Pr}\left(D_{i} \mid H_{j}\right) \operatorname{Pr}\left(H_{j}\right) .
$$

The test statistic can then be a set of likelihood ratios

$$
\begin{aligned}
\Lambda(\mathrm{g}) & =\left[\Lambda_{2}(\mathrm{~g}), \ldots, \Lambda_{L}(\mathrm{~g})\right] \\
\Lambda_{j} & =\frac{\operatorname{pr}\left(\mathrm{g} \mid H_{j}\right)}{\operatorname{pr}\left(\mathrm{g} \mid H_{1}\right)} \quad j=2, \ldots, L .
\end{aligned}
$$


The decision rules correspond to multiple hyperplanes in the $\Lambda_{2}, \ldots, \Lambda_{L}$ plane. The detailed derivation and an example of three-class problem can be found in refs. [48,67]. Some important results are given in ref. [67]:

1. The minimum dimension of the decision space is no more than $L-1$. The boundaries of decision regions are the hyper-surfaces in the $\left(\Lambda_{2}, \ldots, \Lambda_{L}\right)$ space.

2. The optimum test is straightforward to find. However, when we consider specific examples, the error probabilities are frequently difficult to compute.

3. A particular test of importance is the minimum-total-probability-of-error test. Here we compute the posteriori probability of each hypothesis $\operatorname{Pr}\left(H_{j} \mid \mathbf{g}\right)$ and choose the largest.

Some examples can be found in refs. [48,67].

The Hotelling observer can also be extended into the $L$-class problem where $L$ is larger than 2. We first define two scatter matrices, interclass scatter matrix $S_{1}$ and intraclass scatter matrix $S_{2}$ in the $L$-class task:

$$
\begin{aligned}
& \mathrm{S}_{1}=\frac{1}{L} \sum_{l=1}^{L}\left(\overline{\mathbf{g}}-\overline{\mathbf{g}}_{l}\right)\left(\overline{\mathbf{g}}-\overline{\mathbf{g}}_{l}\right)^{t}, \\
& \mathrm{~S}_{2}=\frac{1}{L} \sum_{l=1}^{L}\left\langle\left(\mathrm{~g}-\overline{\mathbf{g}}_{l}\right)\left(\mathrm{g}-\overline{\mathrm{g}}_{l}\right)^{t}\right\rangle_{l},
\end{aligned}
$$

where $\overline{\mathbf{g}}_{l}$ is the ensemble average image vector when the $l^{\text {th }}$ class presents and $\overline{\mathbf{g}}$ is the overall average image vector $\overline{\mathrm{g}}=\frac{1}{L} \sum_{l=1}^{L} \overline{\mathrm{g}}_{l}$. The measure of the class separability in the $L$-class problem is called the Hotelling trace $J_{H o t}$,

$$
J_{H o t}=\operatorname{tr}\left[\mathrm{S}_{2}^{-1} \mathrm{~S}_{1}\right]
$$

The Hotelling trace is a scalar measure involving only the first and second moments of the data, which is subsequently feasible to calculate in most cases. For a binary detection task, the Hotelling trace is reduced to a quarter of the SNR square,

$$
J_{H o t}=\frac{1}{4} \Delta \overline{\mathbf{g}}^{t} \mathrm{~S}_{2}^{-1} \overline{\mathbf{g}}=\frac{1}{4} \mathrm{SNR}_{\text {Hot }}^{2} .
$$


The Hotelling observer is the optimum linear observer that achieves maximum performance among all linear observers in terms of maximizing $J_{H o t}$.

Given the scatter matrices, we can determine the test statistic of the Hotelling observer in the $L$-class problem. The derivation is given in ref. [25]. The key is that we can simultaneously diagonalize two Hermitian matrices by a linear transformation [25]. The simultaneous diagonalizing of $S_{1}$ and $S_{2}$ is as follows,

$$
\mathrm{W}^{\dagger} \mathrm{S}_{2} \mathrm{~W}=\mathrm{I} \quad \mathrm{W}^{\dagger} \mathrm{S}_{1} \mathrm{~W}=\mathrm{D},
$$

where $\mathbf{D}$ is a diagonal matrix. This transformation is equivalent to a eigenvalue problem [9]

$$
\mathrm{S}_{2}^{-1} \mathrm{~S}_{1} \mathrm{~W}=\mathrm{WD}
$$

where $\mathrm{W}$ is the eigenvectors of the product matrix $\mathrm{S}_{2}^{-1} \mathrm{~S}_{1}$ and $\mathrm{D}$ is the eigenvalues in the form of a diagonal matrix. The Hotelling observer uses the vector test statistic $\mathbf{t}$ to classify the data space,

$$
\mathbf{t}=\mathbf{W}^{t} \mathbf{g}
$$

We index the $\mathrm{m}^{\text {th }}$ column vector $\mathrm{w}_{m}$ and the corresponding eigenvalue $\mu_{m}$. Therefore, the $\mathrm{m}^{\text {th }}$ element of the test statistic is

$$
t_{m}=\mathbf{w}_{m}^{t} \mathbf{g}
$$

The strategy of the Hotelling observer to separate the data space into unambiguous parts are more involved in the $L$-class problem. One choice can be to choose the hypothesis that has the maximum probability of getting the given data.

In the binary detection problems, the interclass scatter matrix $\mathrm{S}_{1}$ reduces to

$$
\mathrm{S}_{1}=\frac{1}{4} \Delta \overline{\mathrm{g}} \Delta \overline{\mathrm{g}}^{t}
$$

whose rank is one. The eigenvalue problem (2.1.27) becomes an equation including a single eigenvalue $\mu$

$$
\mathrm{S}_{2}^{-1} \mathrm{~S}_{1} \mathrm{w}=\mu \mathrm{w}
$$


where $\mathrm{w}$ is a vector with the same dimension as the data $\mathrm{g}$. It can be show that the equation suggests that

$$
\mathrm{w}=\mathrm{S}_{2}^{-1} \Delta \mathrm{g}
$$

This is a generalized form for the optimal feature vector of (2.1.9) when the covariances under both hypotheses are unequal. The solution is the same as the original Hotelling observer in binary tasks. With this vector, we can construct the Hotelling observer. The reponse function of the Hotelling observer in detection task is the inner product between the image vector and this vector.

\subsection{Estimation Task}

Estimation tasks can be regarded as the limit of multiple-hypothesis classification tasks where the number of hypotheses goes to uncountably infinite. The treatment is analogous to that of classification tasks in many aspects. Instead of dividing data space into several regions, the observer of estimation tasks assigns values to each point in the data space. The value is the estimate of the parameter we are interested in. The observer, or the estimator, is the rule used to determine the estimate from the observed data.

\subsubsection{Bayesian Observers}

The Bayesian observer minimizes the Bayes risk to get the estimator $\hat{\boldsymbol{\theta}}(\mathrm{g})$ of the set of parameters $\boldsymbol{\theta}$ from the given data $\mathrm{g}$. Corresponding to the prior probabilities in the classification problem, we have the prior probability density $\operatorname{pr}(\theta)$ in the estimation problem. The cost in the classification problem is a set of cost values assigned to each pair of possible decision and truth outcomes. In the estimation problem, the parameter and its estimate are both continuous. The cost is thus a function of the two continuous variables, and we denote it as $C(\boldsymbol{\theta}, \hat{\boldsymbol{\theta}})$. Once the prior probability 
densities and the cost function are specified, we can write an expression for the Bayes risk:

$$
\bar{C}=\int_{\infty} \mathrm{d} \boldsymbol{\theta} \operatorname{pr}(\boldsymbol{\theta})\left\{\int_{\infty} \mathrm{d} \mathbf{g} C[\boldsymbol{\theta}, \hat{\boldsymbol{\theta}}(\mathrm{g})] \operatorname{pr}(\mathrm{g} \mid \boldsymbol{\theta})\right\} .
$$

Different estimators can be formulated by changing cost functions. Three commonly used cost functions are discussed next. The complete discussion can be found in refs. $[9,48,67]$.

For the quadratic cost function

$$
C(\boldsymbol{\theta}, \hat{\boldsymbol{\theta}})=\|\boldsymbol{\theta}-\hat{\boldsymbol{\theta}}\|^{2}=\sum_{i=1}^{I}\left(\theta_{i}-\hat{\theta}_{i}\right)^{2},
$$

the risk corresponds to mean-square error. The estimator is

$$
\hat{\boldsymbol{\theta}}_{M S E}=\int \mathrm{d} \boldsymbol{\theta} \theta \operatorname{pr}(\boldsymbol{\theta} \mid \mathrm{g})
$$

where the subscript $M S E$ is the abbreviation for mean-square error. This is the mean of the posteriori density (or the conditional mean).

For the uniform cost function

$$
C(\boldsymbol{\theta}, \hat{\boldsymbol{\theta}})=1-\operatorname{rect}\left(\frac{\boldsymbol{\theta}-\hat{\boldsymbol{\theta}}}{2 \epsilon}\right),
$$

where $\epsilon$ is small, the estimate satisfies

$$
\hat{\boldsymbol{\theta}}_{M A P}=\arg \max _{\boldsymbol{\theta}} \operatorname{pr}(\boldsymbol{\theta} \mid \mathbf{g}) .
$$

The probability density $\operatorname{pr}(\boldsymbol{\theta} \mid \mathrm{g})$ is the posterior probability of $\boldsymbol{\theta}$ when the data $\mathbf{g}$ are given. Therefore the estimate is called the maximum a posteriori estimation, which is abbreviated in the subscript. $\hat{\boldsymbol{\theta}}_{M A P}$ is the mode of the conditional density $\operatorname{pr}(\boldsymbol{\theta} \mid \mathbf{g})$, so the estimate is also called the conditional mode.

The absolute-value cost function, or the linear cost function

$$
C(\theta, \hat{\theta})=|\theta-\hat{\theta}|
$$


is limited to scalar parameters $\theta$. The estimator is given as

$$
\int_{-\infty}^{\hat{\theta}_{A B S}} \mathrm{~d} \theta \operatorname{pr}(\theta \mid \mathbf{g})=\int_{\hat{\theta}_{A B S}}^{\infty} \mathrm{d} \theta \operatorname{pr}(\theta \mid \mathbf{g}) .
$$

This is just the definition of the median of the posterior density. When generalized to vector cases, the linear cost function does not lead to easily interpreted estimators.

It is shown in ref. [67] that for a large class of cost functions the Bayesian estimate is the $M S E$ estimate whenever the posterior density is a unimodal function which is symmetric about the conditional mean. In many cases the MAP and MSE estimates will be in fact identical.

When the parameter to be estimated is considered non-random or the priori probability density is unknown, we can employ another powerful estimator called maximumlikelihood estimate, or $M L$ estimate. In general, we denote the conditional probability density function $\operatorname{pr}(\mathbf{g} \mid \boldsymbol{\theta})$ as the likelihood function when considering it as a function of $\boldsymbol{\theta}$. The maximum likelihood estimate $\hat{\boldsymbol{\theta}}_{M L}$ is the value of $\boldsymbol{\theta}$ at which the likelihood function is maximized,

$$
\hat{\boldsymbol{\theta}}_{M L}=\arg \max _{\boldsymbol{\theta}} \operatorname{pr}(\mathrm{g} \mid \boldsymbol{\theta})
$$

The maximum likelihood estimate is the limiting case of a MAP estimate when the prior knowledge goes to zero, or the prior density $\operatorname{pr}(\boldsymbol{\theta})$ is sufficiently broad. However, the $M L$ estimate is extremely useful because of its simplicity and the minimum statistical information required.

\subsubsection{Performance Measures}

Bayes risk is one of the figures of merit for estimation tasks. Its definition is found in (2.2.1), where the cost function is averaged over both the noisy data and the random objects. We consider that the data are derived from certain noisy measurements, causing different data at repeated measurement trials on the same object. Addition- 
ally, objects under study are random according to some prior probability densities. This philosophical stance is the basis of the various Bayesian observers derived above.

For a particular true parameter value $\boldsymbol{\theta}$, the mean-square error, or the MSE, of an estimate is defined as the average deviation from the truth conditioned on the given value,

$$
\operatorname{MSE}(\boldsymbol{\theta})=\left\langle\|\hat{\boldsymbol{\theta}}-\boldsymbol{\theta}\|^{2}\right\rangle_{\mathbf{g} \mid \boldsymbol{\theta}} .
$$

Note that the MSE is not the same as the conditional variance because MSE is measured by the deviation from the truth, not the mean estimate. When the estimate is unbiased, or the mean estimate is equal to the truth, the MSE is the same as the conditional variance of the estimate.

When the underlying parameter is itself random, the ensemble mean-square error, or the EMSE, is defined as the averaged MSE over the prior probability density on the parameter,

$$
\mathrm{EMSE}=\left\langle\left\langle\|\hat{\boldsymbol{\theta}}-\boldsymbol{\theta}\|^{2}\right\rangle_{\mathbf{g} \mid \boldsymbol{\theta}}\right\rangle_{\boldsymbol{\theta}} .
$$

The computation of the EMSE requires the prior knowledge of the probability density function of $\operatorname{pr}(\boldsymbol{\theta})$. It can be either an actual sample function or a subjective Bayesian prior. If we define the bias $\mathrm{B}$ as the difference between the mean estimate and the truth,

$$
\mathrm{B}(\theta)=\langle\hat{\theta}\rangle-\theta
$$

the MSE and the EMSE can be written as

$$
\begin{aligned}
\operatorname{MSE}(\boldsymbol{\theta}) & =\operatorname{tr}\left[\mathbf{K}_{\hat{\theta}}\right]+\operatorname{tr}\left[\mathbf{B ~ B}^{\dagger}\right] \\
\mathrm{EMSE} & =\operatorname{tr}\left[\overline{\mathbf{K}}_{\hat{\theta}}\right]+\operatorname{tr}\left[\overline{\mathbf{B}} \overline{\mathbf{B}}^{\dagger}\right]
\end{aligned}
$$

where $\mathbf{K}_{\hat{\boldsymbol{\theta}}}$ is the covariance matrix of the estimate.

An in-depth discussion of estimation tasks can be found in refs. $[9,48,67]$. 


\section{Chapter 3}

\section{System Description AND Modeling}

To apply the fore-mentioned statistical decision theory to real imaging system analysis, we need to know the statistics of the images produced by the system under investigation. A good way to decide the image statistics is to develop a comprehensive statistical description of the imaging chain as the system model and propagate objects through it. Besides the statistical system model, the randomness of objects should be included to complete the image statistics. Building accurate mathematical system models require a sound understanding of underlying physical imaging processes. We will therefore describe all the important parts in a physical system and explain their working principles. The mathematical model is built on the conditions and assumptions drawn from the essential physics. We then apply the statistical decision theory to the mathematical model and derive an analytical expression of the objective image quality. The mathematical expression of image quality is thus used as a figure of merit in system evaluation and optimization. We choose an optically coupled digital mammographic system as an example to illustrate the procedure of applying objective image quality in optical design.

An optically coupled digital mammographic imaging system usually includes an x-ray source, a fluorescent screen, an imaging optical component and a digital image receiver. A layout drawing of the model system is in Fig. 3.1. A remote x-ray generator is placed in front of a fluorescent screen. Patients to be imaged stay between the $\mathrm{x}$-ray source and the screen, usually close to the screen. The detection unit after the screen consists of an optical imaging lens and a CCD camera. The lens images the exit surface of the screen onto the detector plane of the CCD camera. Optimizing the optical imaging part requires the knowledge of the entire system in order to model 
precisely the imaging chain. In the following sections, each component in the model system is carefully investigated and the statistical description of each imaging stage follows. 


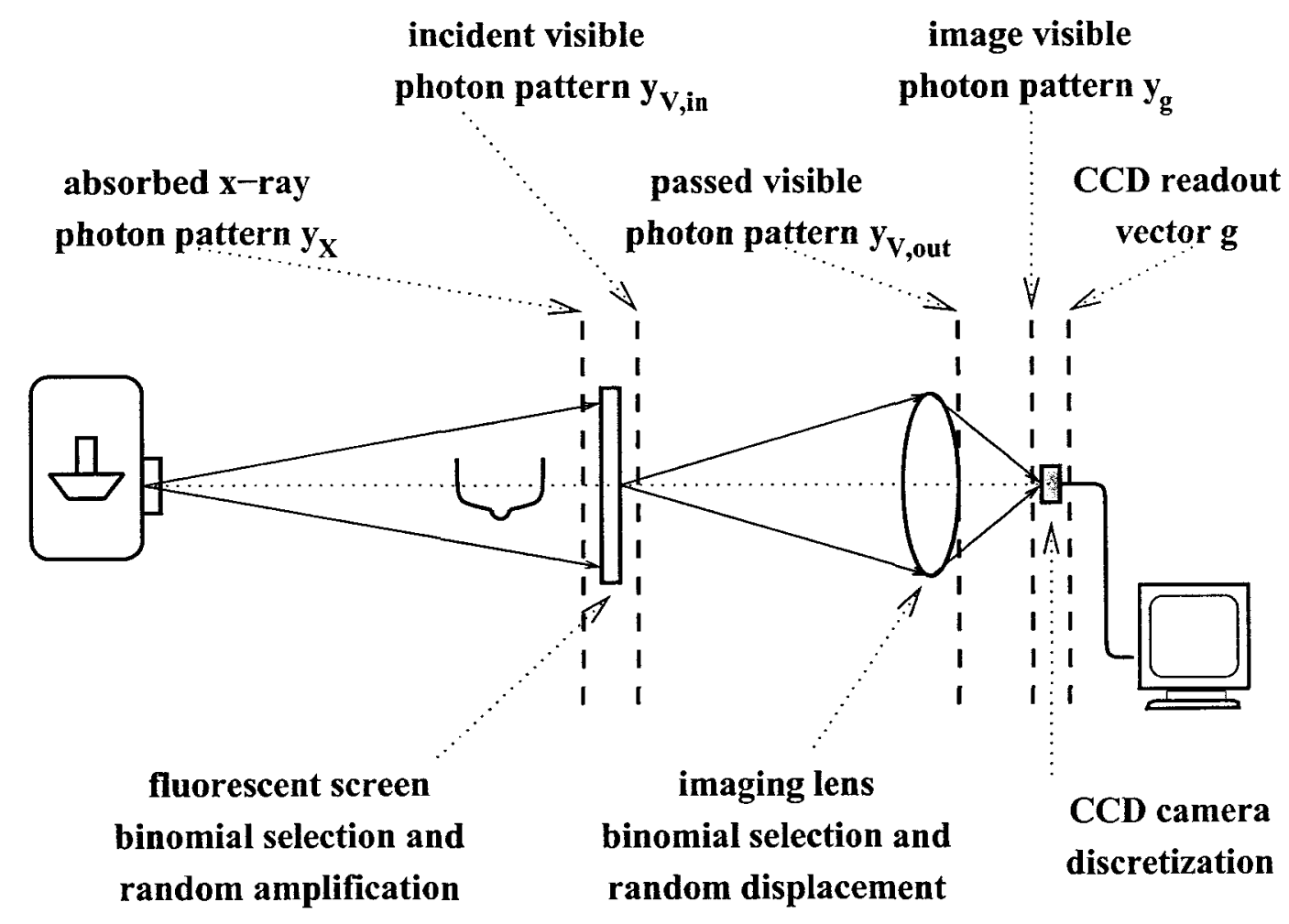

FIGURE 3.1. The layout of the model system 


\subsection{Physical System}

The most common type of $x$-ray source in diagnostic radiology is a vacuum tube in which high-energy electrons bombard a metal anode and create $\mathrm{x}$-ray photons. The electrons interact with the anode metal and lose their energy to the material. The absorbed energy from the electrons mostly appears in the form of heat, but a small amount is transformed into $\mathrm{x}$ rays. If the mean electron current in a tube is constant, the x-ray photons produced are independent of each other, and the total number is Poisson distributed. When a single image is collected for a given patient, a small change in the current for that image is equivalent to a small change in exposure time, the effect of which is negligible. The x-ray photons produced during each exposure are thus independent of each other. When propagating through patients, $\mathrm{x}$-ray photons interact independently with tissue. Some are absorbed, others get out of patients either without interaction or by scattering from tissue. The transmitted $\mathrm{x}$-ray photons after patients are still independent of each other. To increase the image contrast, anti-scatter grids made of a series of parallel lead strips are commonly used between patients and fluorescent screens to remove most of the scattered $\mathrm{x}$ rays after patients. Moreover, because x-ray sources are usually far from fluorescent screens, the $\mathrm{x}$-ray beams are approximately parallel to each other after patients. The detailed model of x-ray source does not come into the mathematical model discussed later. The mathematical model starts just before the fluorescent screen.

$X$-ray fluorescent screens are usually made of rare-earth-doped crystallites embedded in plastic binders. A common type of screen in mammography uses crystalline gadolinium oxysulfide. These tiny crystal grains are activated by terbium, which emits greenish light upon x-ray excitation. Once an x-ray photon is absorbed, some of its energy is then re-emitted in the form of fluorescent light photons. In the diagnostic x-ray spectrum between $20 \mathrm{Kev}$ and $50 \mathrm{Kev}$, each absorbed x-ray photon can produce more than a thousand visible light photons on average. Interactions of 
x-ray photons with fluorescent crystals are unrelated between each other. The initial $\mathrm{x}$-ray interactions with the fluorescent crystals produce high-energy photoelectrons or Compton electrons. A high-energy electron passing through an inorganic scintillator crystallite inside the screen will form a large number of electron-hole pairs by the elevation of electrons from the valence band to the conduction band. The positive hole will quickly drift to the location of a deliberately added impurity site, called an activator, and ionize it, because the ionization energy of the impurity will be less than that of a typical lattice site. The electron will be free to migrate through the crystallite until it encounters such an ionized activator. At this point the electron can drop into the activator site, creating a neutral configuration having its own set of excited energy states within the forbidden band. If the formed activator state is a excited configuration with an allowed transition to the ground state, its de-excitation will occur very quickly and with some probability for the emission of a corresponding visible photon. The photoelectric interaction is dominant in the low energy range like in mammography. A small portion of absorbed $\mathrm{x}$ rays can produce Compton $\mathrm{x}$-ray photons at a lower energy level. Some primary ionized atoms by photoelectric interaction relax radiatively by emitting an Auger X-ray photon. Those secondary $\mathrm{x}$-ray photons can migrate distantly from the first absorption site or even out of the screen without further interaction. The number of the secondary $x$ rays is small and likely blocked by the anti-scattering grid after the screen, so we can neglect their effects now [43]. [9] [55] The e-h pair sites are all so close together near the primary absorption site of the $\mathrm{x}$-ray photon that all the excited visible lights can be considered as emitting from a single $\mathrm{x}$-ray absorption site. All the visible photons generated by a single $x$-ray photon is considered to start from the same absorption site. In fact, the e-h pair range is of order $2 \mu \mathrm{m}$ in the mammographic energy range. After demagnification by the lens, the images of the e-h pair ranges are much smaller than the pixel size in common CCD cameras, which is in the order of tens of microns. But the visible photons are generated by e-h pairs that reach the activators after 
complex migrations, therefore the visible photons carry nearly no information about the initial x-ray photon direction and propagate equally probably in all directions upon creation. Right after generation, visible light beams are scattered inside the screen by these same crystal grains and meander out to the exit surface of the screen. There is also a slight chance for visible light to be absorbed along the scattered path in the screen. Once a visible light photon reaches the exit surface, it is most likely displaced from the original position of the absorbed $\mathrm{x}$-ray photon on the surface and deviated from the original propagation direction of the absorbed x-ray photon. The current screen types usually have a diffusive reflector on their incident sides to direct more visible light out of the exit surface. However, the same reflector increases the scattering spread. The detailed specifications of the screens used in the model are based on Kodak products.

An imaging lens couples the exit surface of the screen onto the front surface of a digital imaging detector. To image the whole area of the screen onto the detector, this lens has to work in a small absolute magnification. The consequently small numerical aperture in the object space cuts off so much visible light emitted from the screen that the ultimate $\mathrm{x}$-ray to light-photon conversion rate at the detector stage is around 1 even though more than 1000 light photons are produced by one $\mathrm{x}$ ray! Making the lens faster while maintaining the field height introduces larger aberrations. Larger aberrations (except distortion) usually further worsen the blur in the final image. Vignetting can help to reduce the off-axis aberrations, but at the same time it causes light loss. The amount of the aberration and the amount of light flux through the lens are coupled with each other.

The last part in the imaging system is a digital imaging detector to produce a discrete set of pixel values representing a continuous image. CCD cameras now have very low noise and very high sensitivity. The electronic noise from CCDs has independent physical sources from other noise introduced before. In the later sections we will find it fairly easy to include any independent noises in the analysis. For now, 
we simulate an ideal CCD camera with an identical response function for all pixels but no electronic noise. The fill factor of the CCD camera is assumed to be 1 .

\subsection{Mathematical Model}

The above detailed descriptions and approximations of the physical model system provide us a solid ground to describe mathematically this imaging chain as a series of cascaded stochastic point processes. This type of model for medical imaging is discussed in detail in [9]. The chain begins just in front of the entrance surface of the fluorescent screen and ends at the CCD camera output.

\subsubsection{Point process of absorbed x-ray photons in the screen}

In radiography the object is the $3 D$ distribution of $\mathrm{x}$-ray attenuation coefficient, and the image is the $2 D$ projection of the $\mathrm{x}$-ray distribution transmitted through that $3 D$ object. To facilitate the point-process analysis later, we use the x-ray distribution transmitted through the patient as the base of the object to our model system. When $\mathrm{x}$-ray beams pass through the fluorescent screen, the crystallites inside the screen absorb a portion of the $\mathrm{x}$ rays. Only the absorbed portion of the $\mathrm{x}$ rays can excite the luminescent crystals to emit visible light and contribute to the image in the CCD camera at last. We therefore take this portion as the object for the model system, and call it $f(\mathbf{R})$, where $\mathbf{R}$ is the $2 D$ coordinate on the entrance surface of the screen. Although a realistic x-ray spectrum will introduce energy-dependent absorption coefficients, we think this detail will not change the results qualitatively: the trends of the SNR curves and the orders of them in the various figures presented later should remain the same, though the exact values can be different. An artificially monochromatic x-ray source is used in our study for the sake of computational simplicity. When we assume a monochromatic x-ray source, the object $f(\mathbf{R})$ can also be interpreted as the mean number of photons per unit area, or the x-ray number 
fluence. The corresponding energy term is call energy fluence, often used in radiology imaging. The energy fluence is defined for a source as the radiant energy per unit area, and this quantity is called exposure in photography. Our object is in fact the noise-free x-ray pattern. If we take into account all the patients to be imaged, the object is a random function sampled from the ensemble of the patients.

Each object produces a noisy x-ray pattern. The pattern fulfills the postulates of Poisson point processes: $\mathrm{x}$-ray photons are independent of each other, and it is almost impossible to produce two $\mathrm{x}$-ray photons at the same time. This x-ray pattern can be described as a two-dimensional Poisson point process, where each x-ray photon contributes a delta function in the random process. The photons all propagate in the same direction which we denote as the $+z$ axis in the $3 D$ coordinate system. The entrance surface of the fluorescent screen therefore lies in the $x y$ plane. Similar to the situation in patients, every $\mathrm{x}$-ray photon interacts independently to all the other photons within the screen. The absorbed portion of the x-ray photons comprises another random point process which also follows Poisson statistics. Mathematically speaking, the absorption mechanism is a binomial selection of the incident point process, and the binomial selection of a Poisson process is still a Poisson with a modified mean [9]. The mean of the Poisson process resulting from the absorption is the object.

Now we are ready to describe the initial random process in the imaging chain. It is a Poisson point process for any given object $f(\mathbf{R})$. This random process models both the object and the noise before the whole imaging system. A sample function of this first random process can be written as

$$
y_{x}(\mathbf{R})=\sum_{n=1}^{N} \delta\left(\mathbf{R}-\mathbf{R}_{n}\right),
$$

where $\mathrm{R}_{n}$ is the $2 D$ position vector in the entrance surface of the screen at which the $n^{\text {th }} \mathrm{x}$-ray photon hits, and each delta function describes a x-ray photon passing through the patient and absorbed by the screen. The x-ray object $f(\mathbf{R})$ is a random 
function drawn from the ensemble of objects to be imaged, so this process is actually a doubly stochastic Poisson process [9]. For any given object, the total number of absorbed $\mathrm{x}$-ray photons $N$ is a Poisson random variable, whose mean is the integral of the process mean $f(\mathbf{R})$. The integral is over the support of the object, which is usually the whole area of the screen,

$$
\bar{N}(f)=\int_{\text {supp }} \mathrm{d} \mathbf{R} f(\mathbf{R}) .
$$

Because of the independence of the photons in the Poisson process, the Probability Density Function (PDF) of $\mathbf{R}_{n}$ is just the properly normalized process mean function,

$$
\operatorname{pr}_{\mathbf{R}}\left(\mathbf{R}_{n} \mid f\right)=\frac{f\left(\mathbf{R}_{n}\right)}{\bar{N}(f)}=\frac{f\left(\mathbf{R}_{n}\right)}{\int_{\text {supp }} \mathrm{d} \mathbf{R} f(\mathbf{R})} .
$$

This $2 D$ point process does not include the third spatial coordinate of the absorbed $\mathrm{x}$-ray photons $z$, which is the absorption depth. The screen should have uniform response over its area when well manufactured. The absorption depth $z_{n}$ of the $n^{\text {th }}$ absorbed x-ray photon is thus independent of its $2 D$ position $\mathbf{R}_{n}$. Given the characteristic x-ray absorption depth $d_{\mathrm{x}}$, the absorption depth $z_{n}$ follows the Beer's law within the screen thickness $d$.

$$
\operatorname{pr}_{Z}\left(z_{n}\right)=\frac{1}{d_{\mathbf{x}}} \cdot \frac{e^{-z_{n} / d_{\mathbf{x}}}}{1-e^{-d / d_{\mathbf{x}}}} \quad 0 \leq z_{n} \leq d .
$$

The proportion of the absorbed $\mathrm{x}$ rays in the $\mathrm{x}$-ray pattern after the patient is $1-e^{-d / d_{\mathbf{x}}}$.

The $2 D$ point process of the absorbed x-ray photons, together with the PDF of the absorption depth, completely describes the first stage in the cascaded imaging system model.

\subsubsection{Point process of incident visible photons on the lens}

Once an x-ray photon is absorbed, some of its energy is then re-emitted in the form of fluorescent light photons. Each absorbed x-ray photon can produce more than a 
thousand visible light photons on average. When secondary $\mathrm{x}$ rays are negligible, all the excited visible lights by a single $\mathrm{x}$ ray can be considered as emitting from a single primary absorption site of the $\mathrm{x}$-ray photon. At the same time, these visible photons propagates equally probably in all directions upon creation. This simplified light conversion model forms the starting point of the visible light scattering simulation thereafter. The visible light photons are scattered by the tiny crystals in the screen and maybe reflected by the diffuse reflectors on the back of the screen. Before they can get out of the screen, they have a slight chance to be absorbed along the way. Because each $\mathrm{x}$-ray photon independently excites scintillation crystallites, the visible photons produced by different $\mathrm{x}$-ray photons are unrelated.

The whole process can be mathematically described as a random amplification process with $\mathrm{x}$-ray photons as primaries and visible photons that get out of the screen as secondaries. The amplification gain of each absorbed x-ray photon is random. The $n^{\text {th }}$ primary produces $K_{n}$ secondaries that come out of the exit surface of the screen, and we can write down a sample function of the $2 D$ stochastic process after this amplification mechanism,

$$
y_{\mathrm{v}, i n}(\mathbf{r})=\sum_{n=1}^{N} \sum_{k=1}^{K_{n}} \delta\left(\mathbf{r}-\mathbf{r}_{n k}\right)=\sum_{n=1}^{N} \sum_{k=1}^{K_{n}} \delta\left(\mathbf{r}-\mathbf{R}_{n}-\Delta \mathbf{r}_{n k}\right) .
$$

where $\mathbf{r}_{n k}$ is the location on the exit surface of the screen for the $k^{\text {th }}$ secondary produced by the $n^{\text {th }}$ primary and $\Delta \mathbf{r}_{n k}$ is the random displacement. The position vectors here and in the rest of the dissertation are all two dimensional. They may be on different planes along the optical axis, but all the planes are parallel to each other and have the identical coordinate systems with the origins on the optical axis. When being projected along the optical axis on a certain plane, position vectors follow the common $2 D$ vector arithmetic correctly.

In radiometry, a function called Bidirectional Transmission Distribution Function $\left.\operatorname{BTDF}\left(\mathbf{r}, \hat{\mathbf{s}}, \hat{\mathbf{s}}^{\prime}\right)\right)$ is used to quantify the directional property of light transmission through a thin transmissive layer [9]. It is defined as the ratio of transmitted radiance 
in the direction $\hat{\mathbf{s}}$ at the position $\mathbf{r}$ to radiant incidence in the direction $\hat{\mathbf{s}}^{\prime}$ on the layer. Radiant incidence is the irradiance of a highly collimated beam traveling in the direction $\hat{\mathbf{s}}^{\prime}$, so BTDF actually specifies the transmitted radiance generated by such a beam. BTDF is in units of $\operatorname{ster}^{-1}$, and we can relate transmitted radiance with incident radiance by the following integral [9],

$$
\mathrm{L}_{t r s}(\mathbf{r}, \hat{\mathbf{s}})=\int_{2 \pi} \mathrm{d} \Omega^{\prime} \operatorname{BTDF}\left(\mathbf{r}, \hat{\mathbf{s}}, \hat{\mathbf{s}}^{\prime}\right) \mathbf{L}_{i n c}\left(\mathbf{r}, \hat{\mathbf{s}}^{\prime}\right) \cos \left(\theta^{\prime}\right),
$$

where the integral is over the hemisphere of unit vector $\hat{\mathbf{s}}^{\prime}$ directed toward the surface, and $\hat{\mathbf{s}}$ lies in the same hemisphere. Here we assume all the visible photons have the same energy. Under the earlier assumption of monochromatic x-ray sources, the radiometric quantities of $\mathrm{x}$ ray and visible light can be defined in units of photon numbers.

Now we generalize the conventional BTDF to describe the entire light transmission through the fluorescent screen. For thick translucent objects like screens, the generalized BTDF is also a function of the position vector on the entrance surface $R$, namely $\operatorname{BTDF}\left(\mathbf{r}, \hat{\mathbf{s}} ; \mathbf{R}, \hat{\mathbf{s}}^{\prime}\right)$. The light-transmission process includes two steps; the first step is the amplification process in which the absorbed $\mathrm{x}$-ray photons are converted into a larger number of visible light photons, and the next step is the scattering process in which the visible light photons are displaced from the original $\mathrm{x}$-ray absorption sites on the exit surface of the screen. In this analysis, the incident light is the absorbed $x$ rays and the transmitted light is the visible light out of the screen. Because the distribution of the transmitted light displacement can be different at different $\mathrm{x}$-ray absorption depths, the generalized BTDF should additionally be a function of the absorption depth $z$. The generalized BTDF is thus similarly defined as the ratio of transmitted radiance in the direction $\hat{\mathbf{s}}$ at the position $\mathbf{r}$ on the exit plane to the radiant flux on the entrance plane when the incident $\mathrm{x}$-ray is absorbed at the depth $z$. Radiant flux is the flux of highly collimated $\mathrm{x}$ rays traveling in direction $\hat{\mathrm{s}}^{\prime}$ with a point-like size at the position $\mathbf{R}$ on the entrance plane. The generalized BTDF, by 
this definition, has units $\mathrm{ster}^{-1} \mathrm{~m}^{-2}$, suggesting that it can be used in an integral over solid angle and two-dimensional spatial vector. In fact, it is the kernel of the integral relating incident radiance to transmitted radiance if the absorption happens at depth $z$

$$
\mathbf{L}_{t r s}(\mathbf{r}, \hat{\mathbf{s}} \mid z)=\int_{2 \pi} \mathrm{d} \Omega^{\prime} \int_{\infty} \mathrm{d} \mathbf{R} \operatorname{BTDF}\left(\mathbf{r}, \hat{\mathbf{s}}, \mathbf{R}, \hat{\mathbf{s}}^{\prime} \mid z\right) \mathbf{L}_{i n c}\left(\mathbf{R}, \hat{\mathbf{s}}^{\prime}\right) \cos \left(\theta^{\prime}\right),
$$

where all the radiometric quantities are in units of photon numbers. Therefore, the mean gain of the amplification process is automatically incorporated into the generalized BTDF.

In our model, the incident radiance is an angular delta function scaled by the absorbed x-ray irradiance. Because we assume x rays incident normally on the screen, the direction cosine is one. The delta function is along the propagating direction $\hat{\mathbf{s}}_{0}^{\prime}$ of all the $\mathrm{x}$-ray photons, which is parallel to the optical axis, defined in the $+\hat{z}$ direction,

$$
\mathbf{L}_{i n c}\left(\mathbf{R}, \hat{\mathbf{s}}^{\prime}\right)=\frac{\mathbf{M}_{i n c}(\mathbf{R}) \delta\left(\hat{\mathbf{s}}^{\prime}-\hat{\mathbf{s}}_{0}^{\prime}\right)}{\cos \left(\theta^{\prime}\right)}=\mathbf{M}_{i n c}(\mathbf{R}) \delta\left(\hat{\mathbf{s}}^{\prime}-\hat{\mathbf{z}}\right),
$$

where $\mathbf{M}_{i n c}$ is the absorbed incident X-ray irradiance on the entrance surface at the position $\mathbf{R}$. By applying (3.2.7), it is easy to get the transmitted visible light radiance at the absorption $\operatorname{depth} z$,

$$
\mathbf{L}_{t r s}(\mathbf{r}, \hat{\mathbf{s}} \mid z)=\int_{\infty} \mathrm{d} \mathbf{R} \mathbf{M}_{i n c}(\mathbf{R}) \operatorname{BTDF}(\mathbf{r}, \hat{\mathbf{s}}, \mathbf{R}, \hat{\mathbf{z}} \mid z) .
$$

The subsequent irradiance on the exit surface can be found by integrating the radiance over all directions in the forward hemisphere,

$$
\begin{aligned}
\mathbf{M}_{t r s}(\mathbf{r} \mid z) & =\int_{2 \pi} \mathrm{d} \Omega \mathbf{L}_{t r s}(\mathbf{r}, \hat{\mathbf{s}} \mid z) \cos (\theta) \\
& =\int_{\infty} \mathrm{d} \mathbf{R} \mathbf{M}_{i n c}(\mathbf{R}) \int_{2 \pi} \mathrm{d} \Omega \operatorname{BTDF}(\mathbf{r}, \hat{\mathbf{s}}, \mathbf{R}, \hat{\mathbf{z}} \mid z) \cos (\theta) .
\end{aligned}
$$

The kernel relating incident irradiance to transmitted irradiance can also be called 
the irradiance point response function (PRF). It is

$$
\operatorname{PRF}(\mathbf{r}, \mathbf{R} \mid z)=\int_{2 \pi} \mathrm{d} \Omega \operatorname{BTDF}(\mathbf{r}, \hat{\mathbf{s}}, \mathbf{R}, \hat{\mathbf{z}} \mid z) \cos (\theta) .
$$

In photon number units, the irradiances are equivalent to the mean processes $f$ of the corresponding random point processes,

$$
\mathbf{M}_{i n c}(\mathbf{R})=f(\mathbf{R}), \quad \mathbf{M}_{t r s}(\mathbf{r} \mid z)=f^{\prime}(\mathbf{r})
$$

The amplification point response function $\mathrm{p}_{\mathrm{d}}$ can be derived from the irradiance point response function in (3.2.11):

$$
\mathrm{p}_{\mathrm{d}}(\mathbf{r}, \mathbf{R} \mid z)=\int_{2 \pi} \mathrm{d} \Omega \operatorname{BTDF}(\mathbf{r}, \hat{\mathbf{s}}, \mathbf{R}, \hat{\mathbf{z}} \mid z) \cos (\theta)=\bar{K}(\mathbf{R} \mid z) \cdot \operatorname{pr}_{\Delta \mathbf{r}}(\Delta \mathbf{r} \mid \mathbf{R}, z) .
$$

As shown in Ref. [9], it determines both the PDF of the displacement position $\operatorname{pr}_{\Delta \mathbf{r}}$ and the average gain $\bar{K}$ by

$$
\begin{aligned}
\operatorname{pr}_{\Delta \mathbf{r}}(\Delta \mathbf{r} \mid \mathbf{R}, z) & =\frac{\mathrm{p}_{\mathrm{d}}(\mathbf{R}+\Delta \mathbf{r}, \mathbf{R} \mid z)}{\bar{K}(\mathbf{R} \mid z)} \\
\bar{K}(\mathbf{R} \mid z) & =\int_{\infty} \mathrm{d} \mathbf{r} \operatorname{p}_{\mathrm{d}}(\mathbf{r}, \mathbf{R} \mid z)
\end{aligned}
$$

where $\bar{K}$ is the average number of secondaries per primary. We also notice that the average number of secondaries per primary is independent of the particular primary, or $n$. Similarly, the point response function in the four-dimensional space (the $2 D$ position and the $2 D$ direction), is the generalized BTDF in photon number units corrected by the direction cosine,

$$
\operatorname{PRF}(\mathbf{r}, \hat{\mathbf{s}}, \mathbf{R}, \hat{\mathbf{z}} \mid z)=\operatorname{BTDF}(\mathbf{r}, \hat{\mathbf{s}}, \mathbf{R}, \hat{\mathbf{z}} \mid z) \cos (\theta)
$$

The joint PDF of the exit position and direction of a scattered secondary photon is thus the normalized $4 D$ point response function,

$$
\operatorname{pr}(\mathbf{r}, \hat{\mathbf{s}} \mid \mathbf{R}, z)=\frac{\operatorname{BTDF}(\mathbf{r}, \hat{\mathbf{s}}, \mathbf{R}, \hat{\mathbf{z}} \mid z) \cos (\theta)}{\bar{K}(\mathbf{R} \mid z)}
$$


The conditional PDF of the outgoing direction can be derived from the joint PDF (3.2.17). The direction is determined by the azimuth angle $\phi$ and the polar angle $\theta$. The differential solid angle in the angular space is in fact the product of the differential azimuth angle $\mathrm{d} \phi$ and the differential cosine of the polar angle $\mathrm{d} \cos (\theta)$. The direction PDF is thus expressed as the joint PDF of the azimuth angle $\phi$ and the cosine of the polar angle $\cos (\theta)$. The conditional PDF of the outgoing direction is expressed as

$$
\begin{aligned}
\operatorname{pr}(\hat{\mathbf{s}} \mid \Delta \mathrm{r}, \mathbf{R}, z) & =\frac{\operatorname{pr}(\mathbf{R}+\Delta \mathbf{r}, \hat{\mathbf{s}} \mid \mathbf{R}, z)}{\operatorname{pr}(\Delta \mathbf{r} \mid \mathbf{R}, z)} \\
& =\frac{\operatorname{BTDF}(\mathbf{R}+\Delta \mathbf{r}, \hat{\mathbf{s}}, \mathbf{R}, \hat{\mathbf{z}} \mid z) \cos (\theta)}{\int_{2 \pi} \mathrm{d} \Omega^{\prime} \operatorname{BTDF}\left(\mathbf{R}+\Delta \mathbf{r}, \hat{\mathbf{s}}^{\prime}, \mathbf{R}, \hat{\mathbf{z}} \mid z\right) \cos \left(\theta^{\prime}\right)},
\end{aligned}
$$

where the vector $\hat{\mathbf{s}}$ denotes two variables $\phi$ and $\cos (\theta)$.

The conditional direction PDF (3.2.18) is completely determined by the generalized BTDF, but the PDF of the gain is still unknown except the mean value. The amplification mechanism removes the independence among the different points in the resultant process because two or more secondaries can be produced by one primary. Shift invariance holds inside the boundary of well-manufactured screens, where the secondary displacement PDF (3.2.14) and the conditional direction PDF (3.2.18) are both independent of the primary position $\mathbf{R}$. The point response function $\mathrm{p}_{\mathrm{d}}(\mathbf{r}, \mathbf{R} \mid z)$ is now only a function of the displacement $\mathbf{r}-\mathbf{R}$ and the depth $z$, and it can be called the point spread function (PSF). The secondaries from different primaries are independently produced since the screen should have no memory from one primary to another. The multivariate density on $\left\{\Delta \mathbf{r}_{n k}\right\}$ is therefore a product of univariate densities on each of the $\Delta \mathbf{r}_{n k}$. The product form is also valid for the multivariate density on $\left\{\left(\Delta \mathbf{r}_{n k}, \hat{\mathbf{s}}_{n k}\right)\right\}$ and the conditional multivariate density on $\left\{\hat{\mathbf{s}}_{n k} \mid \Delta \mathbf{r}_{n k}\right\}$. Similarly, the conditional multivariate density $\operatorname{pr}\left(\left\{\mathbf{r}_{n k}\right\} \mid \mathbf{R}_{n}\right)$ is the product of the univariate densities $\operatorname{pr}\left(\mathbf{r}_{n k} \mid \mathbf{R}_{n}\right)$. 


\subsubsection{Point process of output visible photons from the lens}

The visible light from the screen has to pass through the optical imaging lens after the screen before contributing to the image on the CCD camera. Because of the limited entrance pupil and the complicated vignetting effect of the lens, each visible photon has only a small chance to pass. The passing probability $\operatorname{pass}(\mathbf{R}+\Delta \mathbf{r}, \mathbf{R} \mid z)$ of an output visible photon depends on its exit position $R+\Delta r$ and its primary position $\mathbf{R}$. Based on the probability densities above, the function $\operatorname{pass}(\mathbf{R}+\Delta \mathbf{r}, \mathbf{R} \mid z)$ can be obtained from the generalized BTDF function,

$$
\begin{aligned}
\operatorname{pass}(\mathbf{R}+\Delta \mathbf{r}, \mathbf{R} \mid z) & =\int_{\Omega(\mathbf{R}+\Delta \mathbf{r})} \mathrm{d} \Omega \operatorname{pr}(\hat{\mathbf{s}} \mid \Delta \mathbf{r}, \mathbf{R}, z) \\
& =\frac{\int_{\Omega(\mathbf{R}+\Delta \mathbf{r})} \mathrm{d} \Omega \operatorname{BTDF}(\mathbf{R}+\Delta \mathbf{r}, \hat{\mathbf{s}}, \mathbf{R}, \hat{\mathbf{z}} \mid z) \cos (\theta)}{\int_{2 \pi} \mathrm{d} \Omega \operatorname{BTDF}(\mathbf{R}+\Delta \mathbf{r}, \hat{\mathbf{s}}, \mathbf{R}, \hat{\mathbf{z}} \mid z) \cos (\theta)},
\end{aligned}
$$

where the solid angle $\Omega(\mathbf{R}+\Delta \mathrm{r})$ incorporates only the directions that a visible photon can pass through the lens from the position $\mathbf{R}+\Delta \mathbf{r}$ on the exit surface of the screen. The solid angle is determined by the lens pupil and vignetting at the field $R+\Delta \mathbf{r}$. This binomial selection mechanism needs to be explicitly expressed because the input process is no longer a Poisson process. One sample function of the resultant process is

$$
y_{\mathrm{v}, \text { out }}(\mathbf{r})=\sum_{n=1}^{N} \sum_{k=1}^{K_{n}} \beta_{n k} \delta\left(\mathbf{r}-\mathbf{R}_{n}-\Delta \mathbf{r}_{n k}\right)
$$

where $\beta_{n k}$ is a random variable taking only two values: it is 0 when the $k^{\text {th }}$ visible photon produced by the $n^{\text {th }} \mathrm{x}$-ray photon doesn't pass through the lens, or 1 when the visible photon reaches the CCD camera. The probability law on $\beta_{n k}$ is

$$
\operatorname{Pr}\left(\beta_{n k} \mid \mathbf{R}_{n}, \Delta \mathbf{r}_{n k}, z_{n}\right)=\left\{\begin{array}{ll}
\operatorname{pass}\left(\mathbf{R}_{n}+\Delta \mathbf{r}_{n k}, \mathbf{R}_{n} \mid z_{n}\right) & \beta_{n k}=1 \\
1-\operatorname{pass}\left(\mathbf{R}_{n}+\Delta \mathbf{r}_{n k}, \mathbf{R}_{n} \mid z_{n}\right) & \beta_{n k}=0
\end{array} .\right.
$$

Each visible photon travels independently inside the lens, so the multivariate density on $\left\{\beta_{n k}\right\}$ is the product of the univariate densities on each $\beta_{n k}$. 


\subsubsection{Point process of visible photons in the image}

According to the terminology conventions in lens design, the object plane is the exit surface of the screen and the image plane is the detector plane of the CCD camera when analyzing the imaging process of the lens in our model system. After the lens, the spherical wave from a point on the object plane is not usually changed into a perfect spherical wavefront focusing on the ideal image point on the image plane. Different parts of the output wavefront focus on the different points around the ideal image point. Because of aberrations, each passed visible photon is deviated from the ideal image position on the image plane. For a given photon (or ray) direction, this deviation is deterministic, but the photon directions are random. Thus aberrations translate randomness of the incident directions $\hat{\mathbf{s}}$ starting from the position $\mathbf{r}$ on the exit surface of the screen into randomness of the position displacements $\Delta r^{\prime \prime}$ away from the ideal image position $\mathbf{r}^{\prime \prime}$ on the CCD detector surface. Another $2 D$ spatial stochastic process is produced by this translated randomness [23]. A sample function of this process is

$$
y_{g}^{\prime}\left(\mathbf{r}^{\prime \prime}\right)=\sum_{n=1}^{N} \sum_{k=1}^{K_{n}} \beta_{n k} \delta\left(\mathbf{r}^{\prime \prime}-\mathbf{r}_{n k}^{\prime \prime}\right)
$$

where $\mathbf{r}_{n k}^{\prime \prime}$ is the position on the detector plane of the $k^{\text {th }}$ visible photon produced by the $n^{\text {th }} \mathrm{x}$-ray photon.

Because of the magnification of the lens, ideal image positions are generally not the same as the corresponding object positions. We can properly scale the sample functions on the CCD detector plane to cancel the image magnification. In particular, every position vector is divided by the magnification so that the ideal image points coincide with the corresponding object points. The resultant sample function is expressed as

$$
y_{g}\left(\mathbf{r}^{\prime}\right)=\sum_{n=1}^{N} \sum_{k=1}^{K_{n}} \beta_{n k} \delta\left(\mathbf{r}^{\prime}-\mathbf{r}_{n k}^{\prime}\right)=\sum_{n=1}^{N} \sum_{k=1}^{K_{n}} \beta_{n k} \delta\left(\mathbf{r}^{\prime}-\mathbf{R}_{n}-\Delta \mathbf{r}_{n k}-\Delta \mathbf{r}_{n k}^{\prime}\right),
$$

where $\Delta \mathbf{r}_{n k}^{\prime}$ is the scaled displacement from the scaled ideal image point position 
which is the same as the object point $\mathbf{R}_{n}+\Delta \mathbf{r}_{n k}$. This scaled process again can be treated as a particular random amplification process with the non-random unit gain. The PDF of the displacement $\Delta \mathbf{r}_{n k}^{\prime}$ equals its point response function $\mathrm{p}_{\mathrm{g}}\left(\mathbf{r}^{\prime}, \mathbf{r} \mid \mathbf{R}, z\right)$,

$$
\mathrm{p}_{\mathrm{g}}\left(\mathbf{R}+\Delta \mathbf{r}+\Delta \mathbf{r}^{\prime}, \mathbf{R}+\Delta \mathbf{r} \mid \mathbf{R}, z\right)=\operatorname{pr}_{\Delta \mathbf{r}^{\prime}}\left(\Delta \mathbf{r}^{\prime} \mid \mathbf{R}, \Delta \mathbf{r}, z\right)
$$

The scale displacements are again independent of each other.

We should note that the point response function is not the properly scaled spot diagram from optical design softwares like CODEV or ZEMAX. That spot diagram is generated from the particular incident direction distribution density which is uniform in the directional tangent. Nor can we compute the point response function from the product of the spot diagram and the actual PDF of the direction. Analytically, we can employ the transformation law to get the PDF of $\Delta \mathbf{r}^{\prime}$ from the PDF of the $\hat{\mathbf{s}}$ inside the passing cone $\Omega(\mathbf{R}+\Delta \mathbf{r})$. For a given lens, any displacement $\Delta \mathbf{r}^{\prime}$ depends on the object point position $\mathbf{r}$ and the incident direction $\hat{\mathbf{s}}$ before the lens

$$
\Delta \mathbf{r}^{\prime}=\Delta \mathbf{r}^{\prime}(\hat{\mathbf{s}}, \mathbf{r})=\Delta \mathbf{r}^{\prime}(\hat{\mathbf{s}}, \mathbf{R}+\Delta \mathbf{r})
$$

We should be aware that the conditional direction PDF inside the passing cone is the original PDF normalized by the probability of being inside the cone,

$$
\operatorname{pr}_{\hat{\mathbf{s}}}(\hat{\mathbf{s}} \mid \mathbf{R}, \Delta \mathbf{r}, z)=\frac{\operatorname{BTDF}(\mathbf{R}+\Delta \mathbf{r}, \hat{\mathbf{s}}, \mathbf{R}, \hat{\mathbf{z}} \mid z) \cos (\theta)}{\iint_{\Omega(\mathbf{R}+\Delta \mathbf{r})} \mathrm{d} \Omega^{\prime} \operatorname{BTDF}(\mathbf{R}+\Delta \mathbf{r}, \hat{\mathbf{s}}, \mathbf{R}, \hat{\mathbf{z}} \mid z) \cos \left(\theta^{\prime}\right)} .
$$

When finding the mean value of some function of the displacement $u\left(\Delta \mathbf{r}^{\prime}\right)$, the integral relation between the point spread function $\mathrm{p}_{\mathrm{g}}$ and the conditional direction PDF is

$$
\begin{aligned}
& \iint_{\infty} \mathrm{d}^{2} \Delta r^{\prime} u\left(\Delta \mathbf{r}^{\prime}\right) \operatorname{pr}_{\Delta \mathbf{r}^{\prime}}\left(\Delta \mathbf{r}^{\prime} \mid \mathbf{R}, \Delta \mathbf{r}, z\right) \\
= & \iint_{\Omega(\mathbf{R}+\Delta \mathbf{r})} \mathrm{d} \Omega u\left(\Delta \mathbf{r}^{\prime}(\hat{\mathbf{s}}, \mathbf{r})\right) \operatorname{pr}(\hat{\mathbf{s}} \mid \mathbf{R}, \Delta \mathbf{r}, z) \\
= & \frac{\iint_{\Omega(\mathbf{R}+\Delta \mathbf{r})} \mathrm{d} \Omega u\left(\Delta \mathbf{r}^{\prime}(\hat{\mathbf{s}}, \mathbf{r})\right) \operatorname{BTDF}(\mathbf{R}+\Delta \mathbf{r}, \hat{\mathbf{s}}, \mathbf{R}, \hat{\mathbf{z}} \mid z) \cos (\theta)}{\iint_{\Omega(\mathbf{R}+\Delta \mathbf{r})} \mathrm{d} \Omega^{\prime} \operatorname{BTDF}\left(\mathbf{R}+\Delta \mathbf{r}, \hat{\mathbf{s}}^{\prime}, \mathbf{R}, \hat{\mathbf{z}} \mid z\right) \cos \left(\theta^{\prime}\right)}
\end{aligned}
$$


where the direction $\hat{\mathbf{s}}$ is chosen inside the passing cone $\Omega(\mathbf{R}+\Delta \mathbf{r})$. Whenever the angular space of passed $\hat{\mathbf{s}}$ is mapped onto the $\Delta \mathbf{r}^{\prime}$ space, this integral relation is always true. We do not usually know the analytical expression of $\Delta \mathbf{r}^{\prime}(\hat{\mathbf{s}}, \mathbf{r})$ or $\mathbf{r}^{\prime}(\hat{\mathbf{s}}, \mathbf{r})$, but we can find precisely $\mathbf{r}^{\prime}(\hat{\mathbf{s}}, \mathbf{r})$ with Snell's law and geometric optics. Given a group of randomly emanating photons corresponding to proper direction PDF, computers can trace them through the lens onto the CCD detector plane and automatically produce the requested sample of the above messy PDF of the scaled final displacement on the CCD.

\subsubsection{Random image vector}

The last step is to map this $2 D$ continuous image function $y_{g}\left(\mathbf{r}^{\prime}\right)$ into a $2 D$ discrete image array $g$. Remember that we ignore the electronic noise from the CCD for now, so the final image array is

$$
\begin{aligned}
\mathbf{g} & =\left\{g_{\mathbf{m}}, \mathbf{m}=\{1,1\},\{1,2\}, \ldots,\{M, M\}\right\}, \\
g_{\mathbf{m}} & =\iint_{\text {supp }} \mathrm{d} \mathbf{r}^{\prime} g\left(\mathbf{r}^{\prime}\right) h_{\mathbf{m}}\left(\mathbf{r}^{\prime}\right),
\end{aligned}
$$

where $h_{\mathbf{m}}\left(\mathbf{r}^{\prime}\right)$ is the response function of the $\mathbf{m}^{\text {th }}$ pixel. This readout procedure can be denoted by a discretization operator $\mathcal{D}$.

$$
\begin{aligned}
\mathbf{g} & =\mathcal{D} \mathbf{y}_{g} \\
{\left[\mathcal{D} \mathbf{y}_{g}\right]_{\mathbf{m}} } & =\iint_{\text {supp }} \mathrm{d} \mathbf{r}^{\prime} y_{g}\left(\mathbf{r}^{\prime}\right) h_{\mathbf{m}}\left(\mathbf{r}^{\prime}\right),
\end{aligned}
$$

Under the ideal CCD model, the response function is the $2 D$ rectangle function centered at the corresponding pixel with the size of the pixel. Every response function has the same size, and adjacent response functions have no gap in between. 


\section{Chapter 4}

\section{Merit FunCtion - SNR}

While designing a lens, designers iteratively optimize different lens parameters to find a local minimum of a conventional merit function in lens design softwares. A conventional merit function today is a weighted sum of the squares of many image defects, including aberration coefficients, spot sizes, RMS WFE and/or MTF at one or more locations in the field of view. This kind of merit function is only loosely connected to the image quality. An objective approach to assessment of image quality, or the taskbased imaging quality, must start with a specification of the task and then determine quantitatively how well the task is performed. The quantitative performance should thus be the objective merit function in the scientific and medical lens design. The objective merit function is a figure of merit. Th higher the objective merit function, the better the lens performs. The best design should maximize the objective merit function.

\subsection{Tasks and Observers Model}

The first task of mammographic imaging systems is to help diagnosing breast tumor. It is a classification task, to make the decision between two hypotheses, tumor-present $H_{1}$ and tumor-absent $H_{0}$. An observer of two-hypotheses classification task uses a threshold $t_{c}$ to make decisions on given images. When its test statistic is larger than the threshold, the observer makes tumor-present decision $D_{1}$; otherwise the tumor-absent decision $D_{0}$ is made. The ROC curve is the plot of the true positive fraction $\operatorname{Pr}\left(D_{1} \mid H_{1}\right)$ versus the false positive fraction $\operatorname{Pr}\left(D_{1} \mid H_{0}\right)$ with the threshold as the changing parameter. The curve completely describes the observer's performance. The area under the ROC curve (AUC) is maximized by the ideal observer. Therefore, 
AUC can be used as an quantitative measure of observers performance.

The observers for tumor-detection task are usually radiologists who look at images. Human observer's performance under this task can be measured from psychophysical studies. The study is limited by the resources and time, and often impossible to apply to system designs where a practical system has not been built yet. Based on the previous work in our group [2], the Hotelling observer with a set of channels can satisfactorily predict human observer's performance in a wide range of situations when the tumor profile is deterministic. It applies Hotelling observer's strategy on the channel output of the image. Its performance can be measured by the SNR as follows

$$
\mathrm{SNR}_{c h}^{2}=\Delta \overline{\mathbf{g}}^{t} \mathbf{T}\left(\mathbf{T}^{t} \mathbf{K} \mathbf{T}\right)^{-1} \mathbf{T} \Delta \overline{\mathrm{g}}
$$

The AUC of the channelized Hotelling observer is difficult to get directly. By the central limit theorem, the test statistic $\mathrm{w}_{c h}^{t} \mathrm{Tg}$ is approximately normal-distributed since it is a linear combination of all the image vector elements. The SNR can thus be transformed into the AUC of the observer by the inverse of the monotonic transform in (2.1.14). The human observer's performance can be compared with that of the channelized Hotelling observer by means of AUC. We will use the SNR since a monotonic transformation preserves the order of the observers performance on images produced by different systems. The SNR is the objective merit function to be used in this work.

\subsection{Procedures}

To compute the merit function, we need the first and the second moments of the image vector, $\overline{\mathbf{g}}_{j}$ and $\mathbf{K}_{j}$ under both hypotheses $j=0,1$. We should calculate moments from bottom up, averaging first over the parameter depending on most number of other parameters, and moving up to the dependent parameters next. The last parameter averaged over is the one all the other parameters depend upon. According to the 
relations among all the random variables, the procedure is [10]

1. Average over $\left\{\Delta \mathbf{r}_{n k}^{\prime}\right\}$ for fixed $\mathbf{R}_{n}, z_{n}$ and $\left\{\Delta \mathbf{r}_{n k}\right\}$ with density (3.2.27),

2. average over $\left\{\beta_{n k}\right\}$ for fixed $\mathbf{R}_{n}, z_{n}$ and $\left\{\Delta \mathbf{r}_{n k}\right\}$ with density (3.2.21),

3. average over $\left\{\Delta \mathbf{r}_{n k}\right\}$ for fixed $\mathbf{R}_{n}$ and $z_{n}$ with density (3.2.14),

4. average over $K_{n}$ for fixed $\mathbf{R}_{n}$ and $z_{n}$,

5. average over $z_{n}$ for fixed $f$ with density (3.2.4),

6. average over $\mathbf{R}_{n}$ for fixed $f$ with density (3.2.3),

7. average over $N$ for fixed $f$ with Poisson density law,

8. average over $f$.

We will follow the steps in calculating the mean vectors and the covariance matrices. The detailed derivations are shown in Appendix A and B.

\section{$4.3 \quad$ Formulas}

The mean image vectors and the covariance matrices are given by (An alternative derivation can be found in chapter 11 of Ref. [9]):

$$
\begin{aligned}
\overline{\mathbf{g}} & =\mathcal{D} \mathbf{y}_{g}, \\
\overline{y_{g}}\left(\mathbf{r}^{\prime}\right) & =\left[\mathcal{H}_{1} \bar{f}\right]\left(\mathbf{r}^{\prime}\right), \\
\mathbf{K}_{\mathbf{g}} & =\mathcal{D} K_{g} \mathcal{D}^{\dagger} \\
K_{g}\left(\mathbf{r}_{1}^{\prime}, \mathbf{r}_{2}^{\prime}\right) & =\left[\mathcal{H}_{1} \bar{f}\right]\left(\mathbf{r}_{2}^{\prime}\right) \delta\left(\mathbf{r}_{1}^{\prime}-\mathbf{r}_{2}^{\prime}\right)+\left[\mathcal{H}_{2} \bar{f}\right]\left(\mathbf{r}_{1}^{\prime}, \mathbf{r}_{2}^{\prime}\right)+\left[\mathcal{H}_{1} K_{f} \mathcal{H}_{1}^{\dagger}\right]\left(\mathbf{r}_{1}^{\prime}, \mathbf{r}_{2}^{\prime}\right),
\end{aligned}
$$


where $\mathcal{D}$ is the discretization operator in (3.2.30). The linear operators $\mathcal{H}_{1}$ and $\mathcal{H}_{2}$ are defined as

$$
\begin{aligned}
{\left[\mathcal{H}_{1} \bar{f}\right]\left(\mathbf{r}^{\prime}\right) } & =\int_{\text {supp }} \mathrm{d} \mathbf{R} \bar{f}(\mathbf{R}) \mathrm{p}_{t o t}\left(\mathbf{r}^{\prime}, \mathbf{R}\right) \\
{\left[\mathcal{H}_{1} K_{f} \mathcal{H}_{1}^{\dagger}\right]\left(\mathbf{r}_{1}^{\prime}, \mathbf{r}_{2}^{\prime}\right) } & =\int_{\text {supp supp }} \int_{1} \mathrm{~d} \mathbf{R}_{1} \mathrm{~d} \mathbf{R}_{2} \mathrm{p}_{t o t}\left(\mathbf{r}_{1}^{\prime}, \mathbf{R}_{1}\right) K_{f}\left(\mathbf{R}_{1}, \mathbf{R}_{2}\right) \mathrm{p}_{t o t}\left(\mathbf{r}_{2}^{\prime}, \mathbf{R}_{2}\right) \\
\mathrm{p}_{t o t}\left(\mathbf{r}^{\prime}, \mathbf{R}\right) & =\frac{1}{d_{x}} \int_{0}^{d} \mathrm{~d} z e^{-d / d_{x}} \mathrm{p}_{t o t}\left(\mathbf{r}^{\prime}, \mathbf{R} \mid z\right) \\
{\left[\mathcal{H}_{2} \bar{f}\right]\left(\mathbf{r}_{1}^{\prime}, \mathbf{r}_{2}^{\prime}\right) } & =\frac{1}{d_{x}} \int_{\text {supp }} \mathrm{d} \mathbf{R} \bar{f}(\mathbf{R}) \int_{0}^{d} \mathrm{~d} z e^{-z / d_{x}} \mathrm{Q}(\mathbf{R}, z) \mathrm{p}_{t o t}\left(\mathrm{r}_{1}^{\prime}, \mathbf{R} \mid z\right) \mathrm{p}_{t o t}\left(\mathbf{r}_{2}^{\prime}, \mathbf{R} \mid z\right) \\
\mathrm{p}_{t o t}\left(\mathbf{r}^{\prime}, \mathbf{R} \mid z\right) & =\int_{\infty} \mathrm{d} \mathbf{r} \mathrm{p}_{\mathrm{d}}(\mathbf{r}, \mathbf{R} \mid z) \operatorname{pass}(\mathbf{r}, \mathbf{R} \mid z) \mathrm{p}_{\mathrm{g}}\left(\mathbf{r}^{\prime}, \mathbf{r} \mid \mathbf{R}, z\right) \\
\mathrm{Q}(\mathbf{R}, z) & =\frac{\frac{m^{2}}{\bar{m}^{2}}-\bar{m}}{}
\end{aligned}
$$

where $f$ is the $\mathrm{x}$-ray fluence, $m$ is the random number of visible photons produced by an $\mathrm{x}$-ray photon absorbed at position $\mathbf{R}$ and depth $z$, and $\mathrm{p}_{\mathrm{d}}$, pass and $\mathrm{p}_{\mathrm{g}}$ are defined in (3.2.13), (3.2.19) and (3.2.24) respectively. The conditional total point response function $\mathrm{p}_{t o t}\left(\mathbf{r}^{\prime}, \mathbf{R} \mid z\right)$ at depth $z$ and the overall total point response function $\mathrm{p}_{\text {tot }}\left(\mathrm{r}^{\prime}, \mathbf{R}\right)$ describe the total blur effect on the final image by the whole imaging system.

The mean number of visible photons produced by an absorbed x-ray photon $\bar{m}$ is not necessarily the same as the mean number of secondaries per primary $\bar{K}(\mathbf{R}, z)$ in the incident visible-photon stage. The mean number of secondaries per primary $\bar{K}$ is the average number of light photons generated by an absorbed x-ray photon and going out of the screen, while the mean number of visible photons produced by an absorbed x-ray photon $\bar{m}$ is the average total number of the light photons including both the portion out of the screen and the portion lost inside the screen. Because 
position and depth may affect visible light loss inside the screen due to absorption and light leakage through edges, $K$ is usually a function of position and depth. On the other hand, as long as the scintillation material is homogeneous, $m$ is usually well approximated as independent of positions. Recalling the electron range is of order $2 \mu m$, much thinner than typical fluorescent screens, we can neglect the edge effect and consider typical fluorescent screens homogeneous. The relation between $\bar{m}$ and $\bar{K}$ is specified in Appendix B.

The $Q$ factor is a constant in homogeneous material as well. It is related to the Swank factor $S$ in describing the fluorescent screens in x-ray imaging $[9,63,62,64]$. The Swank factor is defined as the ratio of the squared mean gain and the second moment of the gain, $S=\bar{m}^{2} / \overline{m^{2}}$. The relationship between the $Q$ factor and the Swank factor is found in the following formula:

$$
Q=\frac{1}{S}-\frac{1}{\bar{m}}
$$

The Swank factor of different scintillation materials can be found in the literature [66].

If we denote the $\mathrm{x}$-ray fluence after normal breast tissue as the background $b(\mathbf{r})$, a tumor as an additive x-ray fluence with the profile $s(\mathbf{r})$, the x-ray fluence after tumor-present tissue is $b+s$, the x-ray fluence after tumor-absent tissue $b$. In the statistical decision theory, the tumor is the signal to be detected. The signal profile $s$ is non-random in the Signal-Known-Exactly (SKE) case, while the background $b$ can be random. The ensemble-average object function and the covariance function are $\bar{b}$ and $K_{b}$ under signal-absent hypothesis $H_{0}, \bar{b}+s$ and $K_{b}$ under signal-present hypothesis $H_{1}$. The SNR of the channelized Hotelling observer is readily derived from the formulas above. with all the operators involved being linear, additions can be interchanged with the operators and applied directly to the fluences. Specifically

$$
\begin{aligned}
\mathrm{SNR}_{c h}^{2} & =\Delta \overline{\mathbf{g}}^{t} \mathbf{T}\left(\mathbf{T}^{t} \mathbf{K} \mathbf{T}\right)^{-1} \mathbf{T} \Delta \overline{\mathbf{g}} \\
\Delta \overline{\mathbf{g}} & =\mathcal{D} \Delta \mathbf{y}_{g}=\mathcal{D} \mathcal{H}_{1} \Delta f
\end{aligned}
$$




$$
\begin{aligned}
\Delta f & =s \\
\mathbf{K} & =\mathcal{D}\left[\frac{1}{2}\left(K_{1}+K_{0}\right)\right] \mathcal{D}^{\dagger} \\
\frac{1}{2}\left(K_{1}+K_{0}\right) & =\left[\mathcal{H}_{1}\left(\bar{b}+\frac{1}{2} s\right)\right]\left(\mathrm{r}_{1}^{\prime}\right) \delta\left(\mathbf{r}_{1}^{\prime}-\mathbf{r}_{2}^{\prime}\right)+\left[\mathcal{H}_{2}\left(\bar{b}+\frac{1}{2} s\right)\right]+\mathcal{H}_{1} K_{b} \mathcal{H}_{1}^{\dagger}
\end{aligned}
$$

Expanding the compact expressions, we are able to see the details underneath. The $i^{\text {th }}$ element of the difference of the mean image vector $\Delta \overline{\mathrm{g}}$ is

$$
\begin{aligned}
{[\Delta \overline{\mathrm{g}}]_{i} } & =\int \mathrm{d} \mathbf{r}^{\prime}\left[\mathcal{H}_{1} s\right]\left(\mathbf{r}^{\prime}\right) h_{i}\left(\mathbf{r}^{\prime}\right) \\
& =\int \mathrm{d} \mathbf{R} s(\mathbf{R}) \int \mathrm{d} \mathbf{r}^{\prime} \mathrm{p}_{t o t}\left(\mathbf{r}^{\prime}, \mathbf{R}\right) h_{i}\left(\mathbf{r}^{\prime}\right) .
\end{aligned}
$$

We write the $i j^{\text {th }}$ element of the matrix $\mathbf{K}$ in three terms,

$$
\begin{aligned}
\mathbf{K}_{i j}= & \iint \mathrm{d}^{2} r^{\prime}\left[\mathcal{H}_{1}\left(\bar{b}+\frac{1}{2} s\right)\right]\left(\mathbf{r}^{\prime}\right) h_{i}\left(\mathbf{r}^{\prime}\right) h_{j}\left(\mathbf{r}^{\prime}\right) \\
& +\iiint \int \mathrm{d}^{2} r_{1}^{\prime} \mathrm{d}^{2} r_{2}^{\prime}\left[\mathcal{H}_{2}\left(\bar{b}+\frac{1}{2} s\right)\right]\left(\mathbf{r}_{1}^{\prime}, \mathbf{r}_{2}^{\prime}\right) h_{i}\left(\mathbf{r}_{1}^{\prime}\right) h_{j}\left(\mathbf{r}_{2}^{\prime}\right) \\
& +\iiint \int \mathrm{d}^{2} r_{1}^{\prime} \mathrm{d}^{2} r_{2}^{\prime}\left[\mathcal{H}_{1} K_{b} \mathcal{H}_{1}^{\dagger}\right]\left(\mathbf{r}_{1}^{\prime}, \mathbf{r}_{2}^{\prime}\right) h_{i}\left(\mathbf{r}_{1}^{\prime}\right) h_{j}\left(\mathbf{r}_{2}^{\prime}\right) \\
= & \iint \mathrm{d}^{2} R\left(\bar{b}(\mathbf{R})+\frac{1}{2} s(\mathbf{R})\right) \iint \mathrm{d}^{2} r^{\prime} \mathrm{p}_{t o t}\left(\mathbf{r}^{\prime}, \mathbf{R}\right) h_{i}\left(\mathbf{r}^{\prime}\right) h_{j}\left(\mathbf{r}^{\prime}\right) \\
& +\iint \mathrm{d}^{2} R\left(\bar{b}(\mathbf{R})+\frac{1}{2} s(\mathbf{R})\right) \frac{1}{d_{x}} \int_{0}^{d} \mathrm{~d} z e^{-z / d_{x}} \mathrm{Q}(\mathbf{R}, z) \\
& \cdot \iint \mathrm{d}^{2} r_{1}^{\prime} \mathrm{p}_{t o t}\left(\mathbf{r}_{1}^{\prime}, \mathbf{R} \mid z\right) h_{i}\left(\mathbf{r}_{1}^{\prime}\right) \iint \mathrm{d}^{2} r_{2}^{\prime} \mathrm{p}_{t o t}\left(\mathbf{r}_{2}^{\prime}, \mathbf{R} \mid z\right) h_{j}\left(\mathbf{r}_{2}^{\prime}\right) \\
& +\iiint \int \mathrm{d}^{2} R_{1} \mathrm{~d}^{2} R_{2} K_{b}\left(\mathbf{R}_{1}, \mathbf{R}_{2}\right) \iint \mathrm{d}^{2} r_{1}^{\prime} \mathrm{p}_{t o t}\left(\mathbf{r}_{1}^{\prime}, \mathbf{R}_{1}\right) h_{i}\left(\mathbf{r}_{1}^{\prime}\right) \\
& +\iint \mathrm{d}^{2} r_{2}^{\prime} \mathrm{p}_{t o t}\left(\mathbf{r}_{2}^{\prime}, \mathbf{R}_{2}\right) h_{j}\left(\mathbf{r}_{2}^{\prime}\right) .
\end{aligned}
$$

These two formulas are the bases of the numerical computation developed later. 


\section{Chapter 5}

\section{Characteristic Functional Analysis}

The image quality analysis developed earlier is directly from the probability densities suggested by the underlying medical imaging physics. After some good approximations and mathematical manipulation, we work out the first two moments of images and get the functional form of the SNR. The procedure is routine but not easily applicable to other modalities because almost all the analytic forms of various probability density functions are in need for the average calculation.

Characteristic functions are another road to calculating moments of random variables. It is generally a systematic method but not an easy one. We will generalize the concept of characteristic functions of random vectors to a concept of characteristic functionals of stochastic processes in the following sections.

The use of characteristic functionals is more than another way of calculating moments. Modern imaging systems rely on complicated hardware and sophisticated software. Object statistics are of more interest recently. There are many situations where the image statistics are too difficult to be described analytically while the characteristic functions or the characteristic functionals can be derived mathematically. One example is in Kupinski's work [44], where it is easy to estimate the characteristic functionals and study the object statistics from the estimates. We can investigate various aspects of imaging chains by means of characteristic functions or characteristic functionals. It is viable to optimize design parameters of hardware and softwares by means of characteristic functions or characteristic functionals. 


\subsection{Characteristic Functionals}

Task-based measurement of image quality requires the statistical characteristics of the image vectors. The random image vector $\mathbf{g}$ is fully governed by the probability density function (PDF), denoted pr(g). Equivalently, we could characterize the randomness in image vectors by using the Fourier transform of the PDF or the characteristic function $(\mathrm{CF})$. It is defined as,

$$
\Psi_{\mathbf{g}}(\rho)=\left\langle\exp \left(-2 \pi i \mathbf{g}^{\dagger} \rho\right)\right\rangle_{\mathbf{g}}
$$

where $\rho$ is the Fourier conjugate of $\mathbf{g}, \mathbf{g}^{\dagger} \boldsymbol{\rho}$ is the inner product of $\mathbf{g}$ and $\rho$, and $\langle\cdot\rangle_{\mathbf{g}}$ represents an expectation over $\operatorname{pr}(\mathbf{g})$. Usually objects are continuous in nature, and can be considered as infinite-dimensional vectors in a Hilbert space, or functions of continuous variables. When taking into account the object variability, an object ensemble can be described by a random process $\mathbf{f}$. The characteristic function of the random process $\mathbf{f}$ has a similar definition,

$$
\Psi_{\mathrm{f}}(\boldsymbol{\xi})=\left\langle\exp \left(-2 \pi i \mathbf{f}^{\dagger} \boldsymbol{\xi}\right)\right\rangle_{\mathrm{f}}
$$

where $\boldsymbol{\xi}$ is the infinite-dimensional Fourier conjugate of $\mathbf{f}$. Because $\boldsymbol{\xi}$ is a function, we will call $\Psi_{\mathrm{f}}(\boldsymbol{\xi})$ its characteristic functional (CFl).

\subsection{Transformation Through Imaging Systems}

Knowing the PDF of images is the same as knowing the characteristic function of images. In the situation where the object is random and the imaging system is well defined, the direct derivation of the PDF of images may be difficult because the PDF of objects is hard to get. When objects can be modeled by functions with random parameters, the characteristic functional of objects can be derived from the prior PDF of the parameters. One can start from the characteristic functional of objects and propagate it through the imaging system. The characteristic function of images 
can thus be obtained [17]. Either the moments of images or the full PDF of images is easily calculated from the characteristic function.

A linear, digital imaging system can be mathematically represented by

$$
\mathbf{g}=\mathcal{H} \mathbf{f}+\mathbf{n}
$$

where $\mathcal{H}$ is the system operator which maps a continuous function to a discrete image. The $i^{\text {th }}$ element of the output vector from the system operator is defined as

$$
[\mathcal{H} \mathbf{f}]_{i}=\int \mathrm{d} \mathbf{r} h_{i}(\mathbf{r}) f(\mathbf{r})
$$

where $h_{i}(\mathbf{r})$ is the $i^{\text {th }}$ system response function.

We assume that the mean image $\overline{\mathbf{g}}$ for a fixed object $\mathbf{f}$ is given by

$$
\overline{\mathrm{g}}=\mathcal{H} \mathbf{f}
$$

For this reason, we call $\mathbf{g}$ the noiseless image. Let us assume that we know the CFl of the object distribution $\Psi_{\mathbf{f}}(\xi)$, where the function $\xi$ represents the Fourier conjugate of the function $\mathbf{f}$. The characteristic function of $\bar{g}$ is

$$
\begin{aligned}
\Psi_{\overline{\mathbf{g}}}(\boldsymbol{\xi}) & =\left\langle\exp \left(-2 \pi i \boldsymbol{\rho}^{\dagger} \overline{\mathbf{g}}\right)\right\rangle_{\overline{\mathbf{g}}} \\
& =\left\langle\exp \left(-2 \pi i \boldsymbol{\rho}^{\dagger} \mathcal{H} \mathbf{f}\right)\right\rangle_{\mathbf{f}} \\
& =\left\langle\exp \left(-2 \pi i\left(\mathcal{H}^{\dagger} \boldsymbol{\rho}\right) \mathbf{f}\right)\right\rangle_{\mathbf{f}}
\end{aligned}
$$

The characteristic function of images is equivalent to

$$
\Psi_{\overline{\mathbf{g}}}(\rho)=\Psi_{\mathbf{f}}\left(\mathcal{H}^{\dagger} \rho\right)
$$

Therefore, if the CFl of $\mathbf{f}$ is known, we also know the CF of any linear mapping of $\mathbf{f}$ by simply using the adjoint of the linear operator.

The characteristic function of the image data $\mathbf{g}=\overline{\mathbf{g}}+\mathbf{n}$ is of practical interest, since it accounts for both object variability and noise. Two common noise models are Gaussian noise and Poisson noise. 
The characteristic function for Gaussian noise with zero-mean and covariance matrix $\mathbf{K}$ is known to be Gaussian shaped as well, with the form

$$
\Psi_{\mathbf{n}}(\rho)=\exp \left(-2 \pi^{2} \rho^{\dagger} \mathbf{K} \rho\right)
$$

Because the Gaussian noise is independent of the non-random object being imaged, we know that the PDF of $\mathbf{g}$ is a convolution of the PDF of $\mathbf{n}$, which yields

$$
\Psi_{\mathbf{g}}(\rho)=\Psi_{\mathbf{f}}\left(\mathcal{H}^{\dagger} \rho\right) \Psi_{\mathbf{n}}(\rho)
$$

Poisson noise is conditioned on the mean image as

$$
\operatorname{pr}(\mathbf{g} \mid \overline{\mathbf{g}})=\prod_{m=1}^{M} \exp \left(\bar{g}_{m}\right) \frac{\bar{g}_{m}^{g_{m}}}{g_{m} !}
$$

where $\bar{g}_{m}$ denotes the $m^{\text {th }}$ component of the $M$ vector $\overline{\mathbf{g}}$. The PDF of $\mathbf{g}$ can then be obtained by marginalizing over the object $\overline{\mathbf{g}}$,

$$
\begin{aligned}
\operatorname{pr}(\mathbf{g}) & =\int \mathrm{d} \overline{\mathbf{g}} \operatorname{pr}(\mathbf{g} \mid \overline{\mathbf{g}}) \operatorname{pr}(\overline{\mathbf{g}}) \\
& =\int \mathrm{d} \overline{\mathbf{g}} \operatorname{pr}(\overline{\mathbf{g}}) \prod_{m=1}^{M} \exp \left(-\bar{g}_{m}\right) \frac{\bar{g}_{m}^{g_{m}}}{g_{m} !} .
\end{aligned}
$$

By taking the expectation of the complex exponentials over the probability density function above, we arrive at [17]

$$
\Psi_{\mathbf{g}}(\boldsymbol{\rho})=\int \mathrm{d} \overline{\mathbf{g}} \operatorname{pr}(\overline{\mathbf{g}}) \prod_{m=1}^{M} \exp \left(-\bar{g}_{m}+\bar{g}_{m} e^{-2 \pi i \rho_{m}}\right),
$$

which is close to the CF of $\mathbf{g}$ except that the term in the exponential is not the same. We can relate the above expression to the $\mathrm{CF}$ of $\bar{g}$ by defining a nonlinear operator $\Gamma$ that maps an $M$ vector to another $M$ vector using the following equation for each component $m$,

$$
[\Gamma(\rho)]_{m}=\frac{-1+\exp \left(-2 \pi i \rho_{m}\right)}{-2 \pi i}
$$


Thus we can relate the CF of $\mathbf{g}$ to that of the noiseless CF of $\overline{\mathbf{g}}$ which we previously related to the $\mathrm{CF}$ of $\mathbf{f}$, i.e.,

$$
\Psi_{\mathrm{g}}(\rho)=\Psi_{\overline{\mathrm{g}}}[\Gamma(\rho)]=\Psi_{\mathrm{f}}\left[\mathcal{H}^{\dagger} \Gamma(\rho)\right]
$$

In other words, once we know the CFl for our object models, we are able to use $\mathcal{H}^{\dagger}$ and a known nonlinear operator to determine the CF for our noisy image data.

The channel operator is sometimes used as the last component in an imaging chain to study the human observers' performance $[51,2]$ or to ease the calculation of ideal observers [7]. It is linear, and thus if we want to know the CF of the channel outputs $\mathbf{v}=\mathbf{T g}=\mathbf{T}(\mathcal{H} \mathbf{f}+\mathbf{n})$, we only need the adjoint $\mathbf{T}^{\dagger}$. That is,

$$
\Psi_{\mathbf{v}}(\boldsymbol{\omega})=\Psi_{\mathrm{f}}\left[\mathcal{H}^{\dagger} \Gamma\left(\mathbf{T}^{\dagger} \boldsymbol{\omega}\right)\right]
$$

where $\boldsymbol{\omega}$ is the Fourier conjugate of the filter outputs $\mathbf{v}$.

\subsection{Object Models}

Object randomness is known to have a substantial effect on observer performance and thus should also be included in the system quality evaluation. The randomness of continuous object functions is difficult to quantify. The background model used in our simulated imaging system in chapter 6, clustered lumpy background, is a type of object model. The clustered lumpy background is a generalization of the lumpy background. Lumpy-background models were first developed by Rolland and Barrett [57]. The lumpy-object model is a continuous mathematical phantom designed to synthesize realistic objects such as biological tissue. In 1999, Bochud et al. [14] extended the lumpy object model to synthesize more complicated objects which visually appear like the real objects obtained in mammographic imaging.

Lumpy objects are generated by summing a random number of lump functions placed randomly in the field of view. Typically, Gaussians are used as the lump 
functions, but other lump profiles could be applicable. Mathematically, a lumpy object is represented as

$$
\mathbf{f}=f(\mathbf{r})=\sum_{n=1}^{N} \Lambda\left(\mathbf{r}-\mathbf{c}_{n} \mid a_{n}, s_{n}\right)
$$

where $\mathbf{r}$ is a spatial variable in the two or three-dimensional object space, $\Lambda(\cdot)$ is the lump profile function, $N$ is the number of lumps. For the $n^{\text {th }}$ lump, $\mathbf{c}_{n}$ is the center of the lump function, $a_{n}$ is the magnitude of the lump function, and $s_{n}$ is the parameter controlling the lump width. The number of lumps $N$ is a Poisson-distributed random number with the mean number of $\bar{N}$. Each center $\mathbf{c}_{n}$ is uniformly distributed within the field of view. Both $a_{n}$ and $s_{n}$ are held constant among all the lumps. The random variables $N$ and $\left\{\mathbf{c}_{n}\right\}$ fully determine the randomness of lumpy objects. We can calculate the $\mathrm{CFl}$ for lumpy objects by substituting the definition of a lumpy object into the expression for the CFl:

$$
\begin{aligned}
\Psi_{\mathbf{f}}(\boldsymbol{\xi}) & =\left\langle\exp \left(-2 \pi i \boldsymbol{\xi}^{\dagger} \mathbf{f}\right)\right\rangle_{\mathbf{f}} \\
& =\left\langle\exp \left[-2 \pi i \int \mathrm{d} \mathbf{r} \xi(\mathbf{r}) \sum_{n=1}^{N} \Lambda\left(\mathbf{r}-\mathbf{c}_{n}\right)\right]\right\rangle_{\left\{\mathbf{c}_{n}, N\right\}} \\
& =\left\langle\prod_{n=1}^{N}\left\langle\exp \left[-2 \pi i \int \mathrm{d} \mathbf{r} \xi(\mathbf{r}) \Lambda\left(\mathbf{r}-\mathbf{c}_{n}\right)\right]\right\rangle_{\left\{\mathbf{c}_{n}\right\}}\right\rangle_{N} \\
& =\left\langle\Psi_{\boldsymbol{\Lambda}}(\boldsymbol{\xi})^{N}\right\rangle_{N} \\
& =\exp (-\bar{N}) \sum_{N=0}^{\infty} \frac{\bar{N}^{N}}{N !} \Psi_{\boldsymbol{\Lambda}}(\boldsymbol{\xi})^{N} \\
& =\exp \left\{-\bar{N}\left[1-\Psi_{\boldsymbol{\Lambda}}(\boldsymbol{\xi})\right]\right\},
\end{aligned}
$$

where $\Psi_{\Lambda}(\xi)$ is the characteristic functional of one randomly located lump given by

$$
\Psi_{\Lambda}(\boldsymbol{\xi})=\left\langle\exp \left[-2 \pi i \int \mathrm{d} \mathbf{r} \xi(\mathbf{r}) \Lambda(\mathbf{r}-\mathbf{c})\right]\right\rangle_{\mathbf{c}} .
$$

The $\mathrm{CFl}$ can be used as the starting point of the imaging chain.

Clustered lumpy objects group the lumps around certain cluster centers that are uniformly distributed within the field of view. This is mathematically represented as 


$$
\mathbf{f}=f(\mathbf{r})=\sum_{n=1}^{N} \sum_{k=1}^{K_{n}} \Lambda\left[\left[R_{\phi_{n}}\right]\left(\mathbf{r}-\mathbf{c}_{n}-\Delta_{n k}\right) \mid \alpha\right],
$$

where $N$ is the number of clusters, $K_{n}$ is the number of lumps in the $n^{\text {th }}$ cluster, $\Delta_{n k}$ is the center of the $k^{t h}$ lump function in the $n^{\text {th }}$ cluster, $\phi_{n}$ is the orientation of the lumps in the $n^{\text {th }}$ cluster, and $\alpha$ contains the parameters characterizing the shape of the lumps. The rotation of the lumps is represented by the rotation matrix $R_{\phi_{n}}$. The angle $\phi_{n}$ is uniformly distributed between 0 and $2 \pi . N$ and $\left\{K_{n}\right\}$ are all Poisson distributed with the mean numbers $\bar{N}$ and $\bar{K}$, respectively. The cluster center $\mathbf{c}_{n}$ is uniformly distributed inside the field of view, while the lump centers within this cluster are Gaussian distributed around $\mathbf{c}_{n}$. That is, $\Delta_{n k}$ is Gaussian distributed around $\mathbf{c}_{n}$ with the width $\sigma_{\Delta}$. Bochud uses a two-dimensional asymmetric lump profile defined in polar coordinate as

$$
\Lambda\left(r, \phi \mid \gamma, \beta, l_{x}, l_{y}\right)=\exp \left\{-\gamma r^{\beta} \sqrt{\frac{l_{y}^{2} \cos ^{2}(\phi)+l_{x}^{2} \sin ^{2}(\phi)}{l_{x}^{2} l_{y}^{2}}}\right\} .
$$

This function is chosen to match the power spectrum of a set of normal mammograms. To calculate the CFl for the clustered lumpy object, we rewrite the definition of a clustered lumpy object as

$$
\mathbf{f}=f(\mathbf{r})=\sum_{n=1}^{N} \Omega\left(\mathbf{r}-\mathbf{c}_{n},\left\{\Delta_{n k}\right\}, \phi_{n}, K_{n} \mid \alpha\right)
$$

where

$$
\Omega\left(\mathbf{r}-\mathbf{c}_{n},\left\{\boldsymbol{\Delta}_{n k}\right\}, \phi_{n}, K_{n} \mid \alpha\right)=\sum_{k=1}^{K_{n}} \Lambda\left[\left[R_{\phi_{n}}\right]\left(\mathbf{r}-\mathbf{c}_{n}-\Delta_{n k}\right) \mid \alpha\right]
$$

and $\left\{\Delta_{n k}\right\}$ is a list of $K_{n}$ lump centers relative to the cluster center $\mathbf{c}_{n}$. Equation (5.3.6) is similar to a lumpy object model with a more complicated lump function. Because $\left\{K_{n}\right\}$ are identically independently distributed among all $n$, so are $\left\{\phi_{n}\right\}$, the characteristic functional of $\Omega\left(\mathbf{r}-\mathbf{c}_{n},\left\{\boldsymbol{\Delta}_{n k}\right\}, \phi_{n}\right)$ is independent of the index $n$ 
as in the lumpy object case. We will start the derivative of the $\mathrm{CFl}$ of the clustered lumpy-object model with

$$
\begin{aligned}
\Psi_{\mathbf{f}}(\boldsymbol{\xi}) & =\left\langle\exp \left(-2 \pi i \boldsymbol{\xi}^{\dagger} \mathbf{f}\right)\right\rangle_{\left\{\boldsymbol{\Delta}_{n k}\right\}, K_{n}, \phi_{n}, \mathbf{c}_{n}, N} \\
& =\left\langle\prod_{n=1}^{N} \Psi_{\Omega}(\boldsymbol{\xi})\right\rangle_{N} \\
& =\left\langle\Psi_{\Omega}(\boldsymbol{\xi})^{N}\right\rangle_{N} \\
& =\exp \left\{-\bar{N}\left[1-\Psi_{\boldsymbol{\Omega}}(\boldsymbol{\xi})\right]\right\} .
\end{aligned}
$$

What is left is the characteristic functional $\Psi_{\Omega}(\boldsymbol{\xi})$. It can be calculated as

$$
\begin{aligned}
& \Psi_{\Omega}(\boldsymbol{\xi}) \\
= & \left\langle\exp \left[-2 \pi i \int \mathrm{d} \mathbf{r} \xi(\mathbf{r}) \Omega\left(\mathbf{r}-\mathbf{c}_{n}\right)\right]\right\rangle_{\left\{\boldsymbol{\Delta}_{n k}\right\}, K_{n}, \mathbf{c}_{n}, \phi_{n}} \\
= & \left\langle\prod_{k_{n}=1}^{K_{n}} \exp \left\{-2 \pi i \int \mathrm{d} \mathbf{r} \xi(\mathbf{r}) \Lambda\left[\left[R_{\phi_{n}}\right]\left(\mathbf{r}-\mathbf{c}_{n}-\boldsymbol{\Delta}_{n k}\right)\right]\right\}\right\rangle_{\left\{\boldsymbol{\Delta}_{n k}\right\}, K_{n}, \mathbf{c}_{n}, \phi_{n}} \\
= & \left\langle\left\langle\Psi_{\boldsymbol{\Lambda}}\left(\boldsymbol{\xi} \mid \phi_{n}, \mathbf{c}_{n}\right)^{K_{n}}\right\rangle_{K_{n}}\right\rangle_{\phi_{n}, \mathbf{c}_{n}} \\
= & \left\langle\exp \left\{-\bar{K}\left[1-\Psi_{\Lambda}(\boldsymbol{\xi} \mid \phi, \mathbf{c})\right]\right\}\right\rangle_{\phi, \mathbf{c}},
\end{aligned}
$$

where $\Psi_{\Lambda}(\xi \mid \phi, \mathbf{c})$ is the characteristic functional of a single randomly located lump with cluster center $\mathbf{c}$ and orientation angle $\phi$. The form of $\Psi_{\Lambda}(\cdot)$ is

$$
\Psi_{\Lambda}(\boldsymbol{\xi} \mid \phi, \mathbf{c})=\left\langle\exp \left\{-2 \pi i \int \mathrm{d} \mathbf{r} \xi(\mathbf{r}) \Lambda\left[\left[R_{\phi}\right](\mathbf{r}-\mathbf{c}-\Delta)\right]\right\}\right\rangle_{\Delta}
$$

The characteristic functional of a single lump is similar to the equation (5.3.3) except that we now take the expectation over $\Delta$ instead of $c$. We are now able to write the final expression for the $\mathrm{CFl}$ of a clustered lumpy object model as

$$
\Psi_{\mathbf{f}}(\xi)=\exp \left[-\bar{N}\left(1-\langle\exp \{-\bar{K}[1-\Psi(\boldsymbol{\xi} \mid \mathbf{c}, \phi)]\}\rangle_{\mathbf{c}, \phi}\right)\right]
$$

The interesting regular expressions of both types of objects demonstrate a rule to do the $\mathrm{CFl}$ calculation for objects with multiple layers of sums: each of which contains i.i.d. random variables. 
For a non-negative integer-valued random variable $n$, its probability-generating function is defined as

$$
\mathrm{M}_{n}(\rho)=\left\langle\rho^{n}\right\rangle_{n}=\sum_{n=0}^{\infty} \rho^{n} \operatorname{Pr}(n) .
$$

A discussion about the probability-generating function can be found in Appendix D. In particular, the probability-generating function of a Poisson random variable $N$ is

$$
\mathrm{M}_{\text {Poisson }}(\rho)=\exp [\bar{N}(1-\rho)]
$$

where $\bar{N}$ is the variable mean. We can write the CFl for lumpy objects as

$$
\Psi_{\text {lumpy }}(\boldsymbol{\xi})=\mathrm{M}_{N}\left[\Psi_{\Lambda}(\boldsymbol{\xi})\right]
$$

where $\Psi_{\Lambda}(\xi)$ is the CFl for a randomly located lump given in the equation (5.3.3). After changing variables in the $\mathrm{CFl}$ for a randomly located lump in the clustered lumpy object case, the equation (5.3.10) can be calculated as

$$
\begin{aligned}
\Psi_{\boldsymbol{\Lambda}, \text { clumpy }}(\boldsymbol{\xi}) & =\left\langle\exp \left\{-2 \pi i \int \mathrm{d} \mathbf{r} \xi(\mathbf{r}) \Lambda\left[\left[R_{\phi}\right](\mathbf{r}-\mathbf{c}-\Delta)\right]\right\}\right\rangle_{\Delta} \\
& =\left\langle\exp \left\{-2 \pi i \int \mathrm{d} \mathbf{r} \xi\left[\left[R_{-\phi}\right](\mathbf{r})\right] \Lambda(\mathbf{r}-\mathbf{c}-\Delta)\right\}\right\rangle_{\Delta} \\
& =\left\langle\exp \left\{-2 \pi i \int \mathrm{d} \mathbf{r} \xi\left[\left[R_{-\phi}\right](\mathbf{r})+\mathbf{c}\right] \Lambda(\mathbf{r}-\Delta)\right\}\right\rangle_{\Delta} \\
& =\Psi_{\boldsymbol{\Lambda},-\phi}\left(\boldsymbol{\xi} \circ \mathcal{S}_{\mathbf{c}} \circ \mathcal{R}_{-\phi}\right),
\end{aligned}
$$

where $\mathcal{R}_{\phi}$ is the rotation mapping that rotates a $2 D$ vector counter-clockwise by an angle $\phi, \mathcal{S}_{\mathbf{c}}$ is the shift mapping that moves a $2 D$ vector along $\mathbf{c}, \boldsymbol{\xi}$ denotes the mapping from a $2 D$ vector to a scalar defined by the function $\xi(\mathbf{r})$. The notion $\circ$ represents the cascading of the two associated mappings, the output from the right mapping serving as the input to the left mapping. Because the argument of the innermost mapping is always $r$ in this work, we omit the argument from now on. For instance, an expression of $\mathbf{h} \circ \mathbf{f}$ means $h[f(\mathbf{r})]$ in this work. The special notation adopted here will facilitate the later derivations. similarly, the argument function in 
the above lump $\mathrm{CFl}$ can be expanded out as

$$
\xi \circ \mathcal{S}_{\mathbf{c}} \circ \mathcal{R}_{-\phi}=\xi\left\{S_{\mathbf{c}}\left[R_{-\phi}(\mathbf{r})\right]\right\}=\xi\left[\left[R_{-\phi}\right](\mathbf{r})+\mathbf{c}\right]
$$

The CFl of clustered lumpy objects is thus expressed as

$$
\Psi_{\text {clumpy }}(\boldsymbol{\xi})=\mathrm{M}_{N}\left\{\left\langle\mathrm{M}_{K}\left[\Psi_{\Lambda,-\phi}\left(\boldsymbol{\xi} \circ \mathcal{S}_{\mathrm{c}} \circ \mathcal{R}_{-\phi}\right)\right]\right\rangle_{\mathrm{c}, \phi}\right\}
$$

where $\mathrm{M}_{N}$ and $\mathrm{M}_{K}$ are the probability-generating functions of the number of cluster centers $N$ and the number of lumps $K$ in a cluster respectively. Both of them are Poisson random variables. The result is true for any number distributions of $N$ and $K$ as long as the lumps and the clusters are independent of each other.

We will use these results to derive the CFls at the different stages in our model system.

\subsection{Characteristic Functionals Through the Model Imaging System}

We can calculate the CFls at each stage in our model imaging system because we have all the analytic forms of intermediate stochastic processes together with their statistical descriptions. Instead of using the transformation method discussed earlier, we will directly work out these CFls. A similar analysis of cascaded imaging chain involving probability-generating functions can be found in refs. [53,54].

\subsubsection{CFl of absorbed x-ray photon processes}

The process considered here is not the x-ray number fluence, or the mean process, but the point process of the absorbed $\mathrm{x}$-ray photons discussed in chapter 3 , section 3.2.1. This $2 \mathrm{D}$ stochastic point process is in the form of

$$
y_{\mathbf{x}}(\mathbf{r})=\sum_{n=1}^{N} \delta\left(\mathbf{r}-\mathbf{R}_{n}\right),
$$


where for any given background $\mathbf{b},\left\{\mathbf{r}_{n}\right\}$ are independently identically distributed (i.i.d.) inside the field of view, and $N$ is a Poisson random variable. The complete $\mathrm{CFl}$ of this process is

$$
\begin{aligned}
\Psi_{\mathbf{y}_{\mathbf{x}}}(\boldsymbol{\xi}) & =\left\langle\left\langle\exp \left(-2 \pi i \boldsymbol{\xi}^{\dagger} \mathbf{y}_{\mathbf{x}}\right)\right\rangle_{\mathbf{y}_{\mathbf{x}} \mid \mathbf{b}}\right\rangle_{\mathbf{b}} \\
& =\left\langle\left\langle\exp \left(-2 \pi i \boldsymbol{\xi}^{\dagger} \mathbf{y}_{\mathbf{x}}\right)\right\rangle_{\left\{\mathbf{R}_{n}\right\}, N \mid \mathbf{b}}\right\rangle_{\mathbf{b}}
\end{aligned}
$$

The conditional $\mathrm{CFl}$ is similar to the $\mathrm{CFl}$ of the lumpy objects with a delta function as the lump profile. We can write it as

$$
\begin{aligned}
\Psi_{\mathbf{y}_{\mathbf{x}} \mid \mathbf{b}}(\boldsymbol{\xi}) & =\left\langle\exp \left(-2 \pi i \boldsymbol{\xi}^{\dagger} \mathbf{y}_{\mathbf{x}}\right)\right\rangle_{\mathbf{y}_{\mathbf{x}} \mid \mathbf{b}} \\
& =\left\langle\exp \left[-2 \pi i \int \mathrm{d} \mathbf{r} \xi(\mathbf{r}) \sum_{n=1}^{N} \delta\left(\mathbf{r}-\mathbf{R}_{n}\right)\right]\right\rangle_{\left\{\mathbf{R}_{n}\right\}, N \mid \mathbf{b}} \\
& =\left\langle\prod_{n=1}^{N} \exp \left[-2 \pi i \xi\left(\mathbf{R}_{n}\right)\right]\right\rangle_{\left\{\mathbf{R}_{n}\right\}, N \mid \mathbf{b}} \\
& =\left\langle\langle\exp [-2 \pi i \xi(\mathbf{R})]\rangle_{\mathbf{R} \mid \mathbf{b}}^{N}\right\rangle_{N \mid \mathbf{b}} \\
& =\exp \left\{-\bar{N}\left[1-\Psi_{\delta \mid \mathbf{b}}(\boldsymbol{\xi})\right]\right\}
\end{aligned}
$$

where $\Psi_{\delta \mid \mathbf{b}}(\boldsymbol{\xi})$ is the conditional CFl of randomly located delta lump functions, conditioned on a given object $\mathbf{b}$,

$$
\begin{aligned}
\Psi_{\boldsymbol{\delta} \mid \mathbf{b}}(\boldsymbol{\xi}) & =\left\langle\exp \left(-2 \pi i \boldsymbol{\xi}^{\dagger} \boldsymbol{\delta}\right)\right\rangle_{\boldsymbol{\delta} \mid \mathbf{b}} \\
& =\left\langle\exp \left[-2 \pi i \int \mathrm{d} \mathbf{r}^{\prime} \xi\left(\mathbf{r}^{\prime}\right) \delta\left(\mathbf{r}^{\prime}-\mathbf{r}\right)\right]\right\rangle_{\mathbf{r} \mid \mathbf{b}} \\
& =\langle\exp [-2 \pi i \xi(\mathbf{r})]\rangle_{\mathbf{r} \mid \mathbf{b}} \\
& =\frac{\int \mathrm{d} \mathbf{r} b(\mathbf{r}) \exp [-2 \pi i \xi(\mathbf{r})]}{\int \mathrm{d} \mathbf{r} b(\mathbf{r})}
\end{aligned}
$$

where $b(\mathbf{r})$ is in the number density of $\mathrm{x}$-ray photons. The Poisson postulates imply that the PDF of the position $r$ of each delta function is the normalized mean process $[9]$

$$
\operatorname{pr}_{\mathbf{r}}(\mathbf{r} \mid \mathbf{b})=\frac{b(\mathbf{r})}{\int \mathrm{d} \mathbf{r} b(\mathbf{r})}
$$


and the mean number of delta lumps in a process is the integral of the mean process over the entire support,

$$
\langle N \mid \mathbf{b}\rangle=\int \mathrm{d} \mathbf{r} b(\mathbf{r})
$$

In our system, the signal is deterministic once presented, while the background is random regardless of the existence of the signal. Therefore, the CFl needs only averaging over the background. The clustered lumpy background is used in our simulation, so the complete $\mathrm{CFl}$ in the signal-absent case is

$$
\begin{aligned}
\Psi_{\mathbf{y}_{\mathbf{x}}}(\boldsymbol{\xi}) & =\left\langle\Psi_{\mathbf{y}_{\mathbf{x}} \mid \mathbf{b}}(\boldsymbol{\xi})\right\rangle_{\mathbf{b}} \\
& =\left\langle\exp \left\{-\bar{N}\left[1-\Psi_{\boldsymbol{\delta} \mid \mathbf{b}}(\boldsymbol{\xi})\right]\right\}\right\rangle_{\mathbf{b}} .
\end{aligned}
$$

Remember the mean number of photons is the integral of the number density inside the field of view $\bar{N}=\int \mathrm{d} \mathbf{r} b(\mathbf{r})$, so

$$
\Psi_{\mathbf{y}_{\mathbf{x}}}(\boldsymbol{\xi})=\left\langle\exp \left\{\int \mathrm{d} \mathbf{r} b(\mathbf{r})\left[e^{-2 \pi i \xi(\mathbf{r})}-1\right]\right\}\right\rangle_{\mathbf{b}} .
$$

Following the similar procedure in section 5.3 and being aware of those i.i.d. random variables, we find that the final expression of the CFl of absorbed x-ray photon processes is

$$
\begin{aligned}
\Psi_{\mathbf{y}_{\mathbf{x}}}(\boldsymbol{\xi})= & \left\langle\operatorname { e x p } \left\{\int \mathrm{d} \mathbf{r} \sum_{n=1}^{N} \sum_{k=1}^{K_{n}} \Lambda\left[\left[R_{\phi_{n}}\right]\left(\mathbf{r}-\mathbf{c}_{n}-\boldsymbol{\Delta}_{k n}\right)\right]\right.\right. \\
& \left.\left.\cdot\left[e^{-2 \pi i \xi(\mathbf{r})}-1\right]\right\}\right\rangle_{\left\{\boldsymbol{\Delta}_{n k}\right\},\left\{\mathbf{c}_{n}\right\},\left\{K_{n}\right\},\left\{\phi_{n}\right\}, N} \\
= & \left\langle\left\langle\left\langle\left\langle\prod _ { n = 1 } ^ { N } \prod _ { k = 1 } ^ { K _ { n } } \operatorname { e x p } \left\{\int \mathrm{d} \mathbf{r} \Lambda\left[\left[R_{\phi_{n}}\right]\left(\mathbf{r}-\mathbf{c}_{n}-\boldsymbol{\Delta}_{n k}\right)\right]\right.\right.\right.\right.\right. \\
& \left.\left.\left.\left.\left.\cdot\left[e^{-2 \pi i \xi(\mathbf{r})}-1\right]\right\}\right\rangle_{\left\{\boldsymbol{\Delta}_{n k}\right\}}\right\rangle_{\left\{K_{n}\right\}}\right\rangle_{\left\{\mathbf{c}_{n}, \phi_{n}\right\}}\right\rangle_{N} \\
= & \exp \left[-\bar{N}\left(1-\left\langle\exp \left\{-\bar{K}\left[1-\Psi_{\mathbf{y}_{\mathbf{x}} \mid \mathbf{\Lambda}}(\boldsymbol{\xi})\right]\right\}\right\rangle_{\mathbf{c}, \phi}\right)\right]
\end{aligned}
$$


where $\Psi_{\mathbf{y}_{\mathbf{x}} \mid \mathbf{\Lambda}}(\boldsymbol{\xi})$ is the conditional CFl of the x-ray photon processes on a single lump randomly displaced around the cluster center $\mathbf{c}$.

$$
\Psi_{\mathbf{y}_{\mathbf{x}} \mid \Lambda}(\boldsymbol{\xi})=\left\langle\exp \left\{\int \mathrm{d} \mathbf{r} \Lambda\left[\left[R_{\phi}\right](\mathbf{r}-\mathbf{c}-\Delta)\right]\left[e^{-2 \pi i \xi(\mathbf{r})}-1\right]\right\}\right\rangle_{\Delta} .
$$

The form of $\Psi_{y_{\mathbf{x}} \mid \boldsymbol{\Lambda}}(\xi)$ is similar to (5.4.8) where $\mathrm{b}$ is now only the lump function $\boldsymbol{\Lambda}$, and it is only averaged over the random displacement $\Delta$.

Now we rewrite the CFl for $\mathrm{x}$-ray photons processes with the help of probabilitygenerating functions and the $\mathrm{CFl}$ of a single delta function. The result is another cascaded form and gives insight on the multiple sum layers.

When the $\mathrm{x}$-ray photon processed $\mathrm{y}_{\mathrm{x}}$ is modeled by a clustered lumpy object with only one lump $\Lambda$ randomly located in the field of view,

$$
f_{\mathbf{x}}(\mathbf{r})=\Lambda\left(\left[R_{\phi}\right](\mathbf{r}-\mathbf{c}-\Delta) \mid \alpha\right)
$$

the conditional mean number of $x$-ray photons on the single-lump clustered lumpy object is $\overline{N_{\mathbf{x}}}=\int \mathrm{d} \mathbf{r} \Lambda(\mathbf{r})$. The conditional $\mathrm{CFl} \Psi_{\mathbf{y} \mathbf{x}} \Lambda(\boldsymbol{\xi})$ is

$$
\Psi_{\mathbf{y}_{\mathbf{x}} \mid \mathbf{\Lambda}}(\boldsymbol{\xi})=\left\langle\exp \left(-\overline{N_{\mathrm{x}}}\left\{1-\int \mathrm{d} \mathbf{r} e^{-2 \pi i \xi(\mathbf{r})} \Lambda\left[\left[R_{\phi}\right](\mathbf{r}-\mathbf{c}-\Delta)\right] / \overline{N_{\mathbf{x}}}\right\}\right)\right\rangle_{\Delta}
$$

After rewriting the integral part as

$$
\begin{aligned}
& \int \mathrm{d} \mathbf{r} e^{-2 \pi i \xi(\mathbf{r})} \Lambda\left[\left[R_{\phi}\right](\mathbf{r}-\mathbf{c}-\Delta)\right] / \overline{N_{\mathbf{x}}} \\
= & \frac{\int \mathrm{d} \mathbf{r} e^{-2 \pi i \xi\left(\left[R_{\phi}\right](\mathbf{r})+\mathbf{c}+\Delta\right)} \Lambda(\mathbf{r})}{\int \mathrm{d} \mathbf{r} \Lambda(\mathbf{r})} \\
= & \Psi_{\delta \mid \Lambda}\left(\xi \circ \mathcal{S}_{\Delta+\mathbf{c}} \circ \mathcal{R}_{-\phi}\right),
\end{aligned}
$$

we can write the conditional $\mathrm{CFI}$ as

$$
\Psi_{\mathbf{y}_{\mathbf{x}} \mid \boldsymbol{\Lambda}}(\boldsymbol{\xi})=\left\langle M_{\text {primary } \mid \Lambda}\left[\Psi_{\delta \mid \Lambda}\left(\boldsymbol{\xi} \circ \mathcal{S}_{\Delta+\mathbf{c}} \circ \mathcal{R}_{-\phi}\right)\right]\right\rangle_{\Delta}
$$

where the subscript primary denotes the probability-generating function of the number of primaries which are the absorbed x-ray photons here. Finally, the new form of 
the $\mathrm{CFl}$ for $\mathrm{x}$-ray photon processes is

$$
\Psi_{\mathbf{y}_{\mathbf{x}}}(\boldsymbol{\xi})=\mathrm{M}_{N}\left(\left\langle\mathrm{M}_{K}\left\{\left\langle\mathrm{M}_{\text {primary } \mid \boldsymbol{\Lambda}}\left[\Psi_{\boldsymbol{\delta} \mid \boldsymbol{\Lambda}}\left(\boldsymbol{\xi} \circ \mathcal{S}_{\mathbf{c}+\boldsymbol{\Delta}} \circ \mathcal{R}_{-\phi}\right)\right]\right\rangle_{\Delta}\right\}\right\rangle_{\mathbf{c}, \phi}\right)
$$

where $N$ is the number of cluster centers, $K$ is the number of $\Lambda$ lumps in a cluster, and primary $\mid \Lambda$ is the number of $\mathrm{x}$-ray photons conditioned on a single lump.

The form of $\mathrm{CFl}$ of the $\mathrm{x}$-ray photon processes is very similar to that for the clustered lumpy object itself. The only difference is the innermost characteristic functional being $\Psi_{\mathbf{\Lambda}}$ in the clustered lumpy objects but $\Psi_{\mathbf{y}_{\mathbf{x}} \mid \mathbf{\Lambda}}$ in X-ray photon processes. The cause of the difference is the different forms of objects. The absorbed $\mathrm{x}$-ray photon process is a sum of the displaced delta functions, the mean function of which is the continuous clustered lumpy object. One more sum layer in the absorbed $\mathrm{x}$-ray photon processes produces one more layer in its $\mathrm{CFl}$, which manifests in the expression of $\Psi_{\mathbf{y}_{\mathbf{x}} \mid \boldsymbol{\Lambda}}$ in the equation (5.4.10).

\subsubsection{CFl of incident visible photon processes on the lens}

Some of the x-ray photons through the fluorescent screen are absorbed and converted to visible photons. These visible photons are scattered by the crystallites inside the screen. When coming out of the screen, the visible photons are displaced from the $\mathrm{x}$-ray photon absorption sites. They serve as the incident light on the lens after the screen. The incident visible photon process is denoted as $\mathrm{y}_{\mathrm{v}, \text { in }}$ and written in the form of

$$
y_{\mathrm{v}, i n}(\mathbf{r})=\sum_{n=1}^{N} \sum_{k_{n}=1}^{K_{n}} \delta\left(\mathbf{r}-\mathbf{R}_{n}-\Delta \mathbf{r}_{n k}\right),
$$

where $N$ is the number of absorbed x-ray photons, $K_{n}$ is the number of visible photons generated by the $n^{\text {th }}$ absorbed x-ray photon, $\mathbf{R}_{n}$ is the $2 D$ absorption position of the $n^{\text {th }}$ absorbed x-ray photon, and $\Delta \mathbf{r}_{n k}$ is the $2 D$ displacement of the $k^{\text {th }}$ visible photon 
produced by the $n^{\text {th }}$ absorbed x-ray photon. Its CFl is

$$
\begin{aligned}
\Psi_{\mathbf{y}_{\mathbf{v}, i n}}(\boldsymbol{\xi}) & =\left\langle\left\langle\left\langle\exp \left(-2 \pi i \boldsymbol{\xi}^{\dagger} \mathbf{y}_{\mathbf{v}, i n}\right)\right\rangle_{\mathbf{y}_{\mathbf{v}, i n} \mid \mathbf{y}_{\mathbf{x}}}\right\rangle_{\mathbf{y}_{\mathbf{x}} \mid \mathbf{b}}\right\rangle_{\mathbf{b}} \\
& =\left\langle\left\langle\left\langle\exp \left(-2 \pi i \boldsymbol{\xi}^{\dagger} \mathbf{y}_{\mathbf{v}, i n}\right)\right\rangle_{\left\{\Delta \mathbf{r}_{n k}\right\}, K_{n} \mid \mathbf{R}_{n}}\right\rangle_{\left\{\mathbf{R}_{n}\right\}, N \mid \mathbf{b}}\right\rangle_{\mathbf{b}} .
\end{aligned}
$$

The number of absorbed x-ray photons $N$ is Poisson distributed. The positions $\left\{\mathbf{R}_{n}\right\}$ are i.i.d. uniformly inside the field of view. The displacements $\left\{\Delta \mathrm{r}_{n k}\right\}$ are also i.i.d., and their probability density function is proportional to the point spread function (PSF) of the screen [9].

For a given background $\mathbf{b}$, the conditional CFl is calculated as

$$
\begin{aligned}
\Psi_{\mathbf{y}_{\mathbf{v}, i n} \mid \mathbf{b}}(\boldsymbol{\xi}) & =\left\langle\exp \left(-2 \pi i \boldsymbol{\xi}^{\dagger} \mathbf{y}_{\mathbf{v}, i n}\right)\right\rangle_{\left\{\Delta \mathbf{r}_{n k}\right\},\left\{\mathbf{R}_{n}\right\},\left\{K_{n}\right\}, N} \\
& =\left\langle\exp \left[-2 \pi i \sum_{n=1}^{N} \sum_{k=1}^{K_{n}} \xi\left(\mathbf{R}_{n}+\Delta \mathbf{r}_{n k}\right)\right]\right\rangle_{\left\{\Delta \mathbf{r}_{n k}\right\},\left\{\mathbf{R}_{n}\right\},\left\{K_{n}\right\}, N} \\
& =\left\langle\prod_{n=1}^{N} \prod_{k=1}^{K_{n}} \exp \left[-2 \pi i \xi\left(\mathbf{R}_{n}+\Delta \mathbf{r}_{n k}\right)\right]\right\rangle_{\left\{\Delta \mathbf{r}_{n k}\right\},\left\{\mathbf{R}_{n}\right\},\left\{K_{n}\right\}, N} \\
& =\left\langle\prod_{n=1}^{N}\left\langle\prod_{k=1}^{K_{n}}\left\langle\exp \left[-2 \pi i \xi\left(\mathbf{R}_{n}+\Delta \mathbf{r}_{n k}\right)\right]\right\rangle_{\left\{\Delta \mathbf{r}_{n k}\right\}}\right\rangle_{\left\{K_{n}\right\}}\right\rangle_{\left\{\mathbf{R}_{n}\right\}, N} \\
& =\left\langle\prod_{n=1}^{N}\left\langle\Psi_{\delta \mid \mathrm{P}_{\mathbf{d}}}^{K_{n}}\left(\boldsymbol{\xi} \circ \mathcal{S}_{\left.\mathbf{R}_{n}\right)}\right\rangle_{\left\{K_{n}\right\}}\right\rangle_{\left\{\mathbf{R}_{n}\right\}, N}\right.
\end{aligned}
$$

where $\mathcal{S}$ is the shifting operator, and $\Psi_{\boldsymbol{\delta} \mid \mathrm{p}_{\mathbf{d}}}(\boldsymbol{\xi})$ is the conditional CFl of a randomly located delta function on the PSF of the screen. The form of the conditional CFl of delta function is as follows

$$
\begin{aligned}
\Psi_{\delta \mid \mathbf{p}_{\mathrm{d}}}(\boldsymbol{\xi}) & =\left\langle\exp (-2 \pi i \xi(\Delta \mathbf{r})\rangle_{\{\Delta \mathbf{r}\}}\right. \\
& =\frac{\int \mathrm{d} \Delta \mathbf{r} \exp (-2 \pi i \Delta \mathbf{r}) p_{\mathrm{d}}(\Delta \mathbf{r})}{\int \mathrm{d} \Delta \mathbf{r} \mathrm{p}_{\mathrm{d}}(\Delta \mathbf{r})} .
\end{aligned}
$$

Because the CFl $\Psi_{\delta \mid \mathfrak{p}_{\mathrm{d}}}\left(\boldsymbol{\xi} \circ \mathcal{S}_{\mathbf{R}_{n}}\right)$ is independent of the number of secondaries $K_{n}$, the conditional $\mathrm{CFl}$ for the visible object on a given background is

$$
\begin{aligned}
\Psi_{\mathbf{y}_{\mathbf{v}, i n} \mid \mathbf{b}}(\boldsymbol{\xi}) & =\left\langle\left\langle\mathrm{M}_{\text {secondary }}\left[\Psi_{\boldsymbol{\delta} \mid \mathbf{p}_{\mathbf{d}}}\left(\boldsymbol{\xi} \circ \mathcal{S}_{\mathbf{R}_{n}}\right)\right]\right\rangle_{\left\{\mathbf{R}_{n}\right\}}\right\rangle_{N} \\
& =\left\langle\left\langle\mathrm{M}_{\text {secondary }}\left[\Psi_{\boldsymbol{\delta} \mid \mathbf{p}_{\mathbf{d}}}\left(\boldsymbol{\xi} \circ \mathcal{S}_{\mathbf{R}}\right)\right]\right\rangle_{\mathbf{R}}^{N}\right\rangle_{N} \\
& =\mathrm{M}_{\text {primary }}\left\{\left\langle\mathrm{M}_{\text {secondary }}\left[\Psi_{\delta \mid \mathbf{p}_{\mathbf{d}}}\left(\boldsymbol{\xi} \circ \mathcal{S}_{\mathbf{R}}\right)\right]\right\rangle_{\mathbf{R}}\right\} .
\end{aligned}
$$


The number of primaries in the random amplification process, i.e. the absorbed x-ray photons, follows Poisson distribution. The mean number is given as $\bar{N}=\int \mathrm{d} \mathbf{R} b(\mathbf{R})$. With the probability-generating function of a Poisson random variable known, the above $\mathrm{CFl}$ can be rewritten as

$$
\Psi_{\mathbf{y}_{\mathbf{v}, \text { in }} \mid \mathbf{b}}(\boldsymbol{\xi})=\exp \left[\int \mathrm{d} \mathbf{R}\left\{\mathrm{M}_{\text {secondary }}\left[\Psi_{\delta \mid \mathbf{p}_{\mathrm{d}}}\left(\boldsymbol{\xi} \circ \mathcal{S}_{\mathbf{R}}\right)\right]-1\right\} b(\mathbf{R})\right]
$$

By averaging $\Psi_{\mathbf{y}_{\mathrm{v}, \text { in }} \mid \mathbf{b}}(\boldsymbol{\xi})$ over the random background $\mathrm{b}$, which is a clustered lumpy background here, the final $\mathrm{CFl}$ of the incident visible photon processes is

$$
\begin{aligned}
& \Psi_{\mathbf{y}_{\mathrm{v}, i n}}(\boldsymbol{\xi}) \\
& =\left\langle\Psi_{\mathrm{y}_{\mathrm{v}, \text { in }} \mid \mathrm{b}}(\boldsymbol{\xi})\right\rangle_{\mathrm{b}} \\
& =\left\langle\operatorname { e x p } \left(\int \mathrm{d} \mathbf{R} \sum_{n=1}^{N} \sum_{k=1}^{K_{n}} \Lambda\left[\left[R_{\phi_{n}}\right]\left(\mathbf{R}-\mathbf{c}_{n}-\Delta_{n k}\right)\right]\right.\right. \\
& \left.\left.\cdot\left\{\mathrm{M}_{\text {secondary }}\left[\Psi_{\delta \mid \mathrm{p}_{\mathrm{d}}}\left(\xi \circ \mathcal{S}_{\mathrm{R}}\right)\right]-1\right\}\right)\right\rangle_{\left\{\Delta_{n k}\right\},\left\{K_{n}\right\},\left\{\mathbf{c}_{n}\right\},\left\{\phi_{n}\right\}, N} \\
& =\left\langle\left\langle\left\langle\left\langle\prod _ { n = 1 } ^ { N } \prod _ { k = 1 } ^ { K _ { n } } \operatorname { e x p } \left(\int \mathrm{d} \mathbf{R} \Lambda\left[\left[R_{\phi_{n}}\right]\left(\mathbf{R}-\mathbf{c}_{n}-\Delta_{n k}\right)\right]\right.\right.\right.\right.\right. \\
& \left.\left.\left.\left.\left.\cdot\left\{\mathrm{M}_{\text {secondary }}\left[\Psi_{\delta \mid \mathrm{p}_{\mathrm{d}}}\left(\xi \circ \mathcal{S}_{\mathrm{R}}\right)\right]-1\right\}\right)\right\rangle_{\left\{\Delta_{n k}\right\}}\right\rangle_{\left\{K_{n}\right\}}\right\rangle_{\left\{\mathrm{c}_{n}, \phi_{n}\right\}}\right\rangle_{N} \\
& =\left\langle\left\langle\prod _ { n = 1 } ^ { N } \left\langle\left\langle\operatorname { e x p } \left(\int \mathrm{d} \mathbf{R} \Lambda\left[\left[R_{\phi_{n}}\right]\left(\mathbf{R}-\mathbf{c}_{n}-\Delta\right)\right]\right.\right.\right.\right.\right. \\
& \left.\left.\left.\left.\left.\cdot\left\{\mathrm{M}_{\text {secondary }}\left[\Psi_{\delta \mid \mathrm{p}_{\mathrm{d}}}\left(\xi \circ \mathcal{S}_{\mathrm{R}}\right)\right]-1\right\}\right)\right\rangle_{\Delta}^{K_{n}}\right\rangle_{K_{n}}\right\rangle_{\left\{\mathbf{c}_{n}, \phi_{n}\right\}}\right\rangle_{N} \\
& =\left\langle\left\langle\prod _ { n = 1 } ^ { N } \mathrm { M } _ { K } \left[\left\langle\operatorname { e x p } \left(\int \mathrm{d} \mathbf{R} \Lambda\left[\left[R_{\phi_{n}}\right]\left(\mathbf{R}-\mathbf{c}_{n}-\Delta\right)\right]\right.\right.\right.\right.\right. \\
& \left.\left.\left.\left.\left.\cdot\left\{\mathrm{M}_{\text {secondary }}\left[\Psi_{\delta \mid \mathbf{p}_{\mathbf{d}}}\left(\boldsymbol{\xi} \circ \mathcal{S}_{\mathbf{R}}\right)\right]-1\right\}\right)\right\rangle_{\Delta}\right]\right\rangle_{\left\{\mathbf{c}_{n}, \phi_{n}\right\}}\right\rangle_{N}
\end{aligned}
$$




$$
\begin{aligned}
= & \left\langle\left\langle\mathrm { M } _ { K } \left[\left\langle\operatorname { e x p } \left(\int \mathrm{d} \mathbf{R} \Lambda\left[\left[R_{\phi}\right](\mathbf{R}-\mathbf{c}-\Delta)\right]\right.\right.\right.\right.\right. \\
& \left.\left.\left.\left.\left.\cdot\left\{\mathrm{M}_{\text {secondary }}\left[\Psi_{\delta \mid \mathrm{p}_{\mathrm{d}}}\left(\boldsymbol{\xi} \circ \mathcal{S}_{\mathbf{R}}\right)\right]-1\right\}\right)\right\rangle_{\Delta}\right]\right\rangle_{\mathbf{c}, \phi}^{N}\right\rangle_{N} \\
= & \mathrm{M}_{N}\left\{\left\langle\mathrm { M } _ { K } \left[\left\langle\operatorname { e x p } \left(\int \mathrm{d} \mathbf{R} \Lambda\left[\left[R_{\phi}\right](\mathbf{R}-\mathbf{c}-\Delta)\right]\right.\right.\right.\right.\right. \\
& \left.\left.\left.\left.\left.\cdot\left\{\mathrm{M}_{\text {secondary }}\left[\Psi_{\delta \mid \mathrm{p}_{\mathrm{d}}}\left(\boldsymbol{\xi} \circ \mathcal{S}_{\mathbf{R}}\right)\right]-1\right\}\right)\right\rangle_{\Delta}\right]\right\rangle_{\mathbf{c}, \phi}\right\} \\
= & \mathrm{M}_{N}\left\{\left\langle\mathrm { M } _ { K } \left[\left\langle\operatorname { e x p } \left(\int \mathrm{d} \mathbf{R} \Lambda(\mathbf{R})\right.\right.\right.\right.\right. \\
& \left.\left.\left.\left.\left.\cdot\left\{\mathrm{M}_{\text {secondary }}\left[\Psi_{\delta \mid \mathrm{p}_{\mathrm{d}}}\left(\boldsymbol{\xi} \circ \mathcal{S}_{\left[R_{-\phi}\right](\mathbf{R})+\mathbf{c}+\Delta}\right)\right]-1\right\}\right)\right\rangle_{\Delta}\right]\right\rangle_{\mathbf{c}, \phi}\right\} \\
= & \mathrm{M}_{N}\left[\left\langle\mathrm{M}_{K}\left(\left\langle\mathrm{M}_{\text {primary } \mid \Lambda}\left\{\left\langle\mathrm{M}_{\text {secondary }}\left[\Psi_{\delta \mid \mathbf{p}_{\mathbf{d}}}\left(\boldsymbol{\xi} \circ \mathcal{S}_{\left[R_{-\phi}\right](\mathbf{R})+\mathbf{c}+\Delta}\right)\right]\right\rangle_{\mathbf{R} \mid \mathbf{\Lambda}}\right\}\right\rangle_{\Delta}\right)\right\rangle_{\mathbf{c}, \phi}\right]
\end{aligned}
$$

where the number of primaries is Poisson-distributed, and the cascaded model is described in the previous chapters $[9,18,53]$. Both the number of clusters $N$ and the number of lumps in a cluster $K$ are Poisson-distributed, and the forms of the probability-generating functions $\mathrm{M}_{N}$ and $\mathrm{M}_{K}$ are in (5.3.13). The probability-generating function of the number of primaries also has the same form. We can therefore find the function in the argument of $\mathrm{M}_{K}$ above as

$$
\mathrm{M}_{\text {primary } \mid \mathbf{\Lambda}}\left(\langle\mathbf{t}\rangle_{\mathbf{R} \mid \mathbf{\Lambda}}\right)=\exp \left[\int \mathrm{d} \mathbf{R} \Lambda(\mathbf{R})(t(\mathbf{R})-1)\right] .
$$

where the function $\mathbf{t}$ is expressed as

$$
\mathbf{t}=\mathrm{M}_{\text {secondary }}\left[\Psi_{\delta \mid \mathrm{p}_{\mathbf{d}}}\left(\boldsymbol{\xi} \circ \mathcal{S}_{\left[R_{-\phi}\right](\mathrm{R})+\mathrm{c}+\Delta}\right)\right]
$$

The conditional average over $\mathrm{t}$ and the probability-generating function of the number of primaries are both on an object containing only one lump at the origin.

The only thing unknown is the probability-generating function of the number of secondaries $\mathrm{M}_{\text {secondary. }}$. The number distribution of the secondaries is hard to measure or model. A discussion can be found in refs. $[59,65]$. Because the total energy in the 
secondaries from a single absorbed $\mathrm{x}$-ray photon is much less than the energy of the $\mathrm{x}$-ray photon itself, the first approximation to the number distribution of secondaries is Poisson law.

\subsubsection{CFl of visible photon processes in images}

The point process of the visible photons out of the lens is very similar to the previous one. We skip the output visible photon processes and proceed on to the next process discussed in section 3.2.4. The exit surface of the screen is then imaged onto the CCD detector surface by the imaging lens between the screen and the CCD. The image on the CCD before digitization is another $2 D$ spatial stochastic point process made of visible photons passing through the lens. The process can be expressed as

$$
y_{g}(\mathbf{r})=\sum_{n=1}^{N} \sum_{k_{n}=1}^{K_{n}} \beta_{n k} \delta\left(\mathbf{r}-\mathbf{R}_{n}-\Delta \mathbf{r}_{n k}-\Delta \mathbf{r}_{n k}^{\prime}\right)
$$

where $\Delta \mathbf{r}_{n k}^{\prime}$ is the displacement of the $k^{\text {th }}$ visible photon produced by the $n^{\text {th }}$ absorbed $\mathrm{x}$-ray photon from the original position of this visible photon on the screen, and $\beta_{n k}$ is the indicator of the presence of this visible photon on the CCD. The indicator is 1 when the visible photon passes through the lens and reaches the CCD detector, and it equals 0 otherwise. The $\mathrm{CFl}$ is

$$
\begin{aligned}
\Psi_{\mathbf{y}_{g}}(\boldsymbol{\xi}) & =\left\langle\left\langle\left\langle\left\langle\exp \left(-2 \pi i \boldsymbol{\xi}^{\dagger} \mathbf{y}_{g}\right)\right\rangle_{\mathbf{y}_{g} \mid \mathbf{y}_{\mathbf{v}, i n}}\right\rangle_{\mathbf{y}_{\mathbf{v}, i n} \mid \mathbf{y}_{\mathbf{x}}}\right\rangle_{\mathbf{y}_{\mathbf{x}} \mid \mathbf{b}}\right\rangle_{\mathrm{b}} \\
& =\left\langle\left\langle\left\langle\left\langle\exp \left(-2 \pi i \boldsymbol{\xi}^{\dagger} \mathbf{y}_{g}\right)\right\rangle_{\beta_{n k}, \Delta \mathbf{r}_{n k}^{\prime} \mid \Delta \mathbf{r}_{n k}, \mathbf{R}_{n}}\right\rangle_{\left\{\Delta \mathbf{r}_{n k}\right\}, K_{n} \mid \mathbf{R}_{n}}\right\rangle_{\left\{\mathbf{R}_{n}\right\}, N \mid \mathbf{b}}\right\rangle_{\mathbf{b}} .
\end{aligned}
$$

The indicator $\beta_{n k}$ is a discrete random variable taking only the value of 0 or 1 . Its probability function depends on the input position of the visible photon on the screen $\mathbf{R}_{n}+\Delta \mathbf{r}_{n k}$. The random displacement $\Delta \mathbf{r}_{n k}^{\prime}$ is because of the random propagation direction of visible photon out of the screen. Its probability density function can be obtained by the transformation of the PDF of the propagation direction $\hat{\mathrm{s}}_{n k}$ incident on the lens. The PDF of the unit vector $\hat{\mathbf{s}}$ is given by the generalized BTDF function 
described in the previous chapters, and we denote it as $\operatorname{pr}(\hat{\mathbf{s}} \mid \mathbf{R}+\Delta \mathbf{r})$ for any visible photon produced by an absorbed $x$-ray photon at $\mathbf{R}$ and going out from the position $\mathbf{R}+\Delta \mathbf{r}$ on the screen. As before, the point response function of the lens for the photon at $\mathbf{R}+\Delta \mathbf{r}$ is called $\mathrm{p}_{\mathrm{g}}\left(\Delta \mathbf{r}^{\prime} \mid \mathbf{R}+\Delta \mathbf{r}\right)$. It equals the conditional PDF of $\Delta \mathbf{r}^{\prime}$ on $\mathrm{R}+\Delta \mathrm{r}$. Inherent aberrations of lenses refract rays taking various directions from a single point on an object plane to a number of points on the corresponding image plane. Rays propagating in different directions may hit the same point on the image plane [40]. Based on this transformation from the incident direction $\hat{\mathbf{s}}$ at the entrance pupil of the lens to the displacement $\Delta \mathbf{r}^{\prime}$ on the CCD, we have

$$
\mathrm{p}_{\mathrm{g}}\left(\Delta \mathbf{r}^{\prime} \mid \mathbf{R}+\Delta \mathbf{r}\right)=\sum_{i} \operatorname{pr}\left(\hat{\mathbf{s}}^{(i)}\left(\Delta \mathbf{r}^{\prime}\right) \mid \mathbf{R}+\Delta \mathbf{r}\right)\left|\frac{\partial \Delta \mathbf{r}^{\prime}}{\partial \hat{\mathbf{s}}^{(i)}}\right|^{-1},
$$

where $\hat{\mathbf{s}}^{(i)}\left(\Delta \mathbf{r}^{\prime}\right)$ is the $i^{\text {th }}$ root of the transformation at the given displacement $\Delta \mathbf{r}^{\prime}$, and $\left|\frac{\partial \Delta \mathbf{r}^{\prime}}{\partial \hat{\mathbf{s}}^{(i)}}\right|$ is the absolute value of the determinant of the Jacobian matrix evaluated at $i^{\text {th }}$ root of the transformation. Because the optical transformation from a incident direction to a outgoing image position is deterministic, one direction is mapped unambiguously to only one position. Different roots $\hat{\mathbf{s}}^{(i)}$ occupy distinct subsets of the incident directions without overlapping. Once the angular distribution among those subsets is given, the displacement position distribution is determined. The union of those subsets is the passing solid angle of the lens. The mean of any function $t$ of $\Delta \mathbf{r}^{\prime}$ can thus be done by taking the average over $\hat{\mathrm{s}}$ instead.

$$
\begin{aligned}
\left\langle t\left(\Delta \mathbf{r}^{\prime}\right)\right\rangle_{\Delta \mathbf{r}^{\prime} \mid \mathbf{R}+\Delta \mathbf{r}} & =\int \mathrm{d} \Delta \mathbf{r}^{\prime} t\left(\Delta \mathbf{r}^{\prime}\right) \mathrm{p}_{\mathrm{g}}\left(\Delta \mathbf{r}^{\prime} \mid \mathbf{R}+\Delta \mathbf{r}\right) \\
& =\sum_{i} \int_{\Omega^{(i)}} \mathrm{d} \Omega(\mathbf{R}+\Delta \mathbf{r})^{(i)} t\left[\Delta \mathbf{r}^{\prime}\left(\hat{\mathbf{s}}^{(i)}\right)\right] \operatorname{pr}\left(\hat{\mathbf{s}}^{(i)} \mid \mathbf{R}+\Delta \mathbf{r}\right)\left|\frac{\partial \Delta \mathbf{r}^{\prime}}{\partial \hat{\mathbf{s}}^{(i)}}\right|^{-1} \\
& =\int_{\Omega(\mathbf{R}+\Delta \mathbf{r})} \mathrm{d} \Omega t\left(\Delta \mathbf{r}^{\prime}(\hat{\mathbf{s}})\right) \operatorname{pr}(\hat{\mathbf{s}} \mid \mathbf{R}+\Delta \mathbf{r})
\end{aligned}
$$

where $\Omega(\mathbf{R}+\Delta \mathbf{r})$ is the passing solid angle of the lens at the starting position $\mathbf{R}+\Delta \mathbf{r}$. In our later calculations, the solid angle contains all the incident directions starting 
from $R+\Delta r$ on the screen in which visible photons can pass through the lens and reach the CCD.

For a given background $\mathrm{b}$, the conditional $\mathrm{CFl}$ of the continuous visible image before the CCD camera is

$$
\begin{aligned}
& \Psi_{\mathbf{y}_{\boldsymbol{g}} \mid \mathbf{b}}(\boldsymbol{\xi}) \\
= & \left\langle\exp \left(-2 \pi i \boldsymbol{\xi}^{\dagger} \mathbf{y}_{g}\right)\right\rangle_{\mathbf{y}_{g} \mid \mathbf{b}} \\
= & \left\langle\exp \left[-2 \pi i \sum_{n=1}^{N} \sum_{k=1}^{K_{n}} \beta_{n k} \xi\left(\mathbf{R}_{n}+\Delta \mathbf{r}_{n k}+\Delta \mathbf{r}_{n k}^{\prime}\right)\right]\right\rangle_{\left\{\beta_{n k}\right\},\left\{\Delta \mathbf{r}_{n k}^{\prime}\right\},\left\{\Delta \mathbf{r}_{n k}\right\},\left\{K_{n}\right\},\left\{\mathbf{R}_{n}\right\}, N} \\
= & \left\langle\left\langle\left\langle\left\langle\left\langle\left\langle\prod_{n=1}^{N} \prod_{k=1}^{K_{n}} \exp \left[-2 \pi i \beta_{n k} \xi\left(\mathbf{R}_{n}+\Delta \mathbf{r}_{n k}+\Delta \mathbf{r}_{n k}^{\prime}\right)\right]\right.\right.\right.\right.\right.\right. \\
& \rangle_{\left\{\beta_{n k}\right\}}\right\rangle_{\left\{\Delta \mathbf{r}_{n k}^{\prime}\right\}}\right\rangle_{\left\{\Delta \mathbf{r}_{n k}\right\}}\right\rangle_{\left\{K_{n}\right\}}\right\rangle_{\left\{\mathbf{R}_{n}\right\}}\right\rangle_{N}
\end{aligned}
$$

The probability of $\beta_{n k}$ taking the value 1 can be denoted as $\operatorname{pass}\left(\mathbf{R}_{n}+\Delta \mathbf{r}_{n k}\right)$. Its probability-generating function is

$$
\mathrm{M}_{\beta_{n k}}(\rho)=(\rho-1) \operatorname{pass}\left(\mathbf{R}_{n}+\Delta \mathbf{r}_{n k}\right)+1
$$

Because all $\beta_{n k}$ are i.i.d., we have

$$
\begin{aligned}
& \left\langle\exp \left(-2 \pi i \boldsymbol{\xi}^{\dagger} \mathbf{y}_{g}\right)\right\rangle_{\left\{\beta_{n k}\right\}} \\
= & \prod_{n=1}^{N} \prod_{k=1}^{K_{n}}\left\langle\exp \left[-2 \pi i \beta_{n k} \xi\left(\mathbf{R}_{n}+\Delta \mathbf{r}_{n k}+\Delta \mathbf{r}_{n k}^{\prime}\right)\right]\right\rangle_{\left\{\beta_{n k}\right\}} \\
= & \prod_{n=1}^{N} \prod_{k=1}^{K_{n}}\left\{\exp \left[-2 \pi i \xi\left(\mathbf{R}_{n}+\Delta \mathbf{r}_{n k}+\Delta \mathbf{r}_{n k}^{\prime}\right)\right] \operatorname{pass}\left(\mathbf{R}_{n}+\Delta \mathbf{r}_{n k}\right)+1\right\}
\end{aligned}
$$

When taking the average over $\Delta \mathbf{r}_{n k}^{\prime}$, only the exponential part contains $\Delta \mathbf{r}_{n k}^{\prime}$,

$$
\begin{aligned}
& \left\langle\exp \left[-2 \pi i \xi\left(\mathbf{R}_{n}+\Delta \mathbf{r}_{n k}+\Delta \mathbf{r}_{n k}^{\prime}\right)\right]\right\rangle_{\Delta \mathbf{r}_{n k}^{\prime}} \\
= & \frac{\int_{2 \pi} \mathrm{d} \Omega_{n k} e^{-2 \pi i \xi\left(\mathbf{R}_{n}+\Delta \mathbf{r}_{n k}+\Delta \mathbf{r}_{n k}^{\prime}\left(\hat{\mathbf{s}}_{n k}\right)\right)} \operatorname{pr}\left(\hat{\mathbf{s}}_{n k} \mid \mathbf{R}_{n}+\Delta \mathbf{r}_{n k}\right) A_{\Omega}\left(\hat{\mathbf{s}}_{n k} \mid \mathbf{R}_{n}+\Delta \mathbf{r}_{n k}\right)}{\int_{2 \pi} \mathrm{d} \Omega_{n k} \operatorname{pr}\left(\hat{\mathbf{s}}_{n k} \mid \mathbf{R}_{n}+\Delta \mathbf{r}_{n k}\right) A_{\Omega}\left(\hat{\mathbf{s}}_{n k} \mid \mathbf{R}_{n}+\Delta \mathbf{r}_{n k}\right)} .
\end{aligned}
$$


With the help of the passing solid angle $\Omega(\mathbf{R}+\Delta \mathbf{r})$, the probability is

$$
\operatorname{pass}\left(\mathbf{R}_{n}+\Delta \mathbf{r}_{n k}\right)=\int_{\Omega\left(\mathbf{R}_{n}+\Delta \mathbf{r}_{n k}\right)} \mathrm{d} \Omega_{n k} \operatorname{pr}\left(\hat{\mathbf{s}}_{n k} \mid \mathbf{R}_{n}+\Delta \mathbf{r}_{n k}\right) .
$$

We can thus get the following expression:

$$
\begin{aligned}
& \left\langle\left\langle\exp \left[-2 \pi i \boldsymbol{\xi}^{\dagger} \mathbf{y}_{g}\right]\right\rangle_{\left\{\beta_{n k}\right\}}\right\rangle_{\left\{\Delta \mathbf{r}_{n k}^{\prime}\right\}} \\
= & \prod_{n=1}^{N} \prod_{k=1}^{K_{n}}\left(\left\{\left\langle\exp \left[-2 \pi i \xi\left(\mathbf{R}_{n}+\Delta \mathbf{r}_{n k}+\Delta \mathbf{r}_{n k}^{\prime}\right)\right]\right\rangle_{\Delta \mathbf{r}_{n k}^{\prime}}\right\} \operatorname{pass}\left(\mathbf{R}_{n}+\Delta \mathbf{r}_{n k}\right)+1\right) \\
= & \prod_{n=1}^{N} \prod_{k=1}^{K_{n}}\left[1+\int_{\Omega\left(\mathbf{R}_{n}+\Delta \mathbf{r}_{n k}\right)} \mathrm{d} \Omega\left(\exp \left\{-2 \pi i \xi\left[\mathbf{R}_{n}+\Delta \mathbf{r}_{n k}+\Delta \mathbf{r}^{\prime}(\hat{\mathbf{s}})\right]\right\}-1\right)\right. \\
& \left.\cdot \operatorname{pr}\left(\hat{\mathbf{s}} \mid \mathbf{R}_{n}+\Delta \mathbf{r}_{n k}\right)\right] \\
= & \prod_{n=1}^{N} \prod_{k=1}^{K_{n}} W\left(\mathbf{R}_{n}+\Delta \mathbf{r}_{n k}\right),
\end{aligned}
$$

where we define a new function $W(\mathbf{r})$ as

$$
W(\mathbf{r})=1+\int_{\Omega(\mathbf{r})} \mathrm{d} \Omega\left\{\exp \left[-2 \pi i \xi\left(\mathbf{r}+\Delta \mathbf{r}^{\prime}(\hat{\mathbf{s}})\right)\right]-1\right\} \operatorname{pr}(\hat{\mathbf{s}} \mid \mathbf{r})
$$

Moving one level up in the nested average, we have

$$
\begin{aligned}
\left\langle\left\langle\left\langle\exp \left[-2 \pi i \boldsymbol{\xi}^{\dagger} \mathbf{y}_{g}\right]\right\rangle_{\left\{\beta_{n k}\right\}}\right\rangle_{\left\{\Delta \mathbf{r}_{n k}^{\prime}\right\}}\right\rangle_{\left\{\Delta \mathbf{r}_{n k}\right\}} & =\prod_{n=1}^{N} \prod_{k=1}^{K_{n}}\left[\frac{\int \mathrm{d} \Delta \mathbf{r} W\left(\mathbf{R}_{n}+\Delta \mathbf{r}\right) \mathrm{p}_{\mathrm{d}}(\Delta \mathbf{r})}{\int \mathrm{d} \Delta \mathbf{r} \mathrm{p}_{\mathrm{d}}(\Delta \mathbf{r})}\right] \\
& =\prod_{n=1}^{N} \widetilde{W}\left(\mathbf{R}_{n}\right)^{K_{n}}
\end{aligned}
$$

where the function $\widetilde{W}(\mathbf{R})$ is defined as

$$
\widetilde{W}(\mathbf{R})=\langle W(\mathbf{R}+\Delta \mathbf{r})\rangle_{\Delta \mathbf{r}}=\frac{\int \mathrm{d} \Delta \mathbf{r} W(\mathbf{R}+\Delta \mathbf{r}) \mathrm{p}_{\mathrm{d}}(\Delta \mathbf{r})}{\int \mathrm{d} \Delta \mathbf{r} \mathrm{p}_{\mathrm{d}}(\Delta \mathbf{r})}
$$

Now we are ready to write out the conditional CFl of the visible photon processes in 
images,

$$
\begin{aligned}
\Psi_{\mathbf{y}_{g} \mid \mathbf{b}}(\boldsymbol{\xi}) & =\left\langle\left\langle\left\langle\prod_{n=1}^{N} \widetilde{W}\left(\mathbf{R}_{n}\right)^{K_{n}}\right\rangle_{\left\{K_{n}\right\}}\right\rangle_{\left\{\mathbf{R}_{n}\right\}}\right\rangle_{N} \\
& =\left\langle\prod_{n=1}^{N}\left\langle\mathrm{M}_{\text {secondary }}[\widetilde{W}(\mathbf{R})]\right\rangle_{\mathbf{R}}\right\rangle_{N} \\
& =\left\langle\left\langle\mathrm{M}_{\text {secondary }}(\widetilde{W}(\mathbf{R}))\right\rangle_{\mathbf{R}}^{N}\right\rangle_{N} \\
& =\mathrm{M}_{\text {primary }}\left[\left\langle\mathrm{M}_{\text {secondary }}(\widetilde{W}(\mathbf{R}))\right\rangle_{\mathbf{R}}\right] \\
& =\exp \left(\int \mathrm{d} \mathbf{R}\left\{\mathrm{M}_{\text {secondary }}[\widetilde{W}(\mathbf{R})]-1\right\} b(\mathbf{R})\right)
\end{aligned}
$$

where the number of primaries is Poisson-distributed.

With the clustered lumpy background and a similar derivation as in the previous section, we can find the final $\mathrm{CFl}$ of the visible photon processes in images as

$$
\begin{aligned}
& \Psi_{\mathbf{y}_{g}}(\boldsymbol{\xi}) \\
= & \left\langle\Psi_{\mathbf{y}_{g} \mid \mathbf{b}}(\boldsymbol{\xi})\right\rangle_{\mathbf{b}} \\
= & \left\langle\exp \left(\int \mathrm{d} \mathbf{R}\left\{\mathrm{M}_{\text {secondary }}[\widetilde{W}(\mathbf{R})]-1\right\} b(\mathbf{R})\right)\right\rangle_{\mathbf{b}} \\
= & \left\langle\operatorname { e x p } \left\{\int \mathrm{d} \mathbf{R} \sum_{n=1}^{N} \sum_{k=1}^{K_{n}} \Lambda\left[\left[R_{\phi_{n}}\right]\left(\mathbf{R}-\mathbf{c}_{n}-\Delta_{n k}\right)\right]\right.\right. \\
& \left.\left.\cdot\left[\mathrm{M}_{\text {secondary }}(\widetilde{W}(\mathbf{R}))-1\right]\right\}\right\rangle_{\left\{\Delta_{n k}\right\},\left\{K_{n}, \mathbf{c}_{n}, \phi_{n}\right\}, N} \\
= & \mathrm{M}_{N}\left[\left\langle\mathrm { M } _ { K } \left(\left\langle\operatorname { e x p } \left\{\int \mathrm{d} \mathbf{R} \Lambda\left[\left[R_{\phi}\right](\mathbf{R}-\mathbf{c}-\Delta)\right]\right.\right.\right.\right.\right. \\
& \left.\left.\left.\left.\left.\cdot\left[\mathrm{M}_{\text {secondary }}(\widetilde{W}(\mathbf{R}))-1\right]\right\}\right\rangle_{\Delta}\right)\right\rangle_{\mathbf{c}, \phi}\right] \\
= & \mathrm{M}_{N}\left(\left\langle\mathrm { M } _ { K } \left\{\left\langle\operatorname { e x p } \left[\int \mathrm{d} \mathbf{R} \Lambda(\mathbf{R})\right.\right.\right.\right.\right. \\
& \left.\left.\left.\left.\left.\cdot\left(\mathrm{M}_{\text {secondary }}\left\{\widetilde{W}\left[\left[R_{-\phi}\right](\mathbf{R})+\mathbf{c}+\Delta\right]\right\}-1\right)\right]\right\rangle_{\Delta}\right\}\right\rangle_{\mathbf{c}, \phi}\right) \\
= & \mathrm{M}_{N}\left\{\left\langle\mathrm{M}_{K}\left[\left\langle\mathrm{M}_{\text {primary } \mid \mathbf{\Lambda}}\left(\left\langle\mathrm{M}_{\text {secondary }}\left\{\widetilde{W}\left[\left[R_{-\phi}\right](\mathbf{R})+\mathbf{c}+\Delta\right]\right\}\right\rangle_{\mathbf{R} \mid \mathbf{\Lambda}}\right)\right\rangle_{\Delta}\right]\right\rangle_{\mathbf{c}, \phi}\right\} .
\end{aligned}
$$


When looking into the expression of $\widetilde{W}(\mathbf{R})$, we can rewrite it in terms of the conditional $\mathrm{CFl}$ of a randomly located delta function on the point response function $\mathrm{p}_{\mathrm{g}}$ of the lens:

$$
\begin{aligned}
& \widetilde{W}(\mathbf{R}) \\
= & \langle W(\mathbf{R}+\Delta \mathbf{r})\rangle_{\Delta \mathbf{r}} \\
= & \left\langle\int_{\Omega(\mathbf{R}+\Delta \mathbf{r})} \mathrm{d} \Omega\left(\exp \left\{-2 \pi i \xi\left[\mathbf{R}+\Delta \mathbf{r}+\Delta \mathbf{r}^{\prime}(\hat{\mathbf{s}})\right]\right\}-1\right) \operatorname{pr}(\hat{\mathbf{s}} \mid \mathbf{R}+\Delta \mathbf{r})\right\rangle_{\Delta \mathbf{r}} \\
= & \left\langle\mathrm{M}_{\beta}\left[\Psi_{\boldsymbol{\delta} \mid \mathbf{p}_{\mathbf{g}}}\left(\boldsymbol{\xi} \circ \mathcal{S}_{\mathbf{R}+\Delta \mathbf{r}}\right)\right]\right\rangle_{\Delta \mathbf{r}} .
\end{aligned}
$$

The CFI of the visible image can be rewritten as

$$
\begin{aligned}
& \Psi_{\mathbf{y}_{g}}(\boldsymbol{\xi}) \\
= & \mathrm{M}\left\{\left\langle\mathrm { M } _ { K } \left[\left\langle\mathrm { M } _ { \text { primary } | \boldsymbol { \Lambda } } \left(\left\langle\mathrm { M } _ { \text { secondary } } \left\{\left\langle\mathrm{M}_{\beta}\left[\Psi_{\boldsymbol{\delta} \mid \mathrm{p}_{\mathbf{g}}}\left(\boldsymbol{\xi} \circ \mathcal{S}_{\left[R_{-\phi}\right](\mathbf{R})+\Delta \mathbf{r}+\mathbf{c}+\Delta}\right)\right]\right.\right.\right.\right.\right.\right.\right.\right. \\
& \rangle_{\Delta \mathbf{r}}\right\}\right\rangle_{\mathbf{R} \mid \boldsymbol{\Lambda}}\right)\right\rangle_{\Delta}\right]\right\rangle_{\mathbf{c}, \phi}\right\},
\end{aligned}
$$

where the probability-generating function of $\beta$ is found in (5.4.30).

\subsubsection{CF of image vectors}

The discrete image vector $\mathbf{g}$ can be given by a linear mapping of the visible photon processes images. The mapping is represented as

$$
\begin{aligned}
\mathbf{g} & =\mathcal{D}_{\mathbf{y}_{g}}, \\
g_{\mathbf{m}} & =\int \mathrm{d} \mathbf{r} y_{g}(\mathbf{r}) h_{\mathbf{m}}(\mathbf{r}), \quad \mathbf{m}=\{1,1\},\{1,2\}, \ldots,\{M, M\} .
\end{aligned}
$$

where $\mathcal{D}$ is the linear discretization operator mapping a function into an $M D$ vector, and $h_{\mathrm{m}}(\mathbf{r})$ is the response function of the $\mathbf{m}^{\text {th }}$ pixel used in the definition of this discretization operator. The characteristic function of the digital image vector can then be directly computed from the characteristic functional of the visible photon processes in images.

$$
\Psi_{\mathbf{g}}(\rho)=\Psi_{\mathbf{y}_{g}}\left(\mathcal{D}^{\dagger} \rho\right) .
$$

The CF of the digital image is calculated with the adjoint of the discretization operator from the $\mathrm{CFl}$ of the visible photon processes in images. 


\subsubsection{Results for lumpy backgrounds}

The CFls for lumpy backgrounds at each imaging stage are given here. The notations are the same as in the clustered-lumpy-background case.

$$
\begin{aligned}
\Psi_{\mathbf{y}_{\mathbf{x}}}(\boldsymbol{\xi}) & =\mathrm{M}_{N}\left\{\left\langle\mathrm{M}_{\text {primary }}\left[\Psi_{\boldsymbol{\delta} \mid \boldsymbol{\Lambda}}\left(\boldsymbol{\xi} \circ \mathcal{S}_{\mathbf{c}}\right)\right]\right\rangle_{\mathbf{c}}\right\} \\
\Psi_{\mathbf{y}_{\mathbf{v}, i n}}(\boldsymbol{\xi}) & =\mathrm{M}_{N}\left(\left\langle\mathrm{M}_{\text {primary } \mid \boldsymbol{\Lambda}}\left\{\left\langle\mathrm{M}_{\text {secondary }}\left[\Psi_{\delta \mid \mathbf{p}_{\mathbf{d}}}\left(\boldsymbol{\xi} \circ \mathcal{S}_{\mathbf{R}+\mathbf{c}}\right)\right]\right\rangle_{\mathbf{R} \mid \mathbf{\Lambda}}\right\}\right\rangle_{\mathbf{c}}\right) \\
\Psi_{\mathbf{y}_{g}}(\boldsymbol{\xi}) & =\mathrm{M}_{N}\left[\left\langle\mathrm{M}_{\text {primary } \mid \boldsymbol{\Lambda}}\left(\left\langle\mathrm{M}_{\text {secondary }}\left\{\left\langle\mathrm{M}_{\beta}\left[\Psi_{\delta \mid \mathbf{p}_{\mathbf{g}}}\left(\boldsymbol{\xi} \circ \mathcal{S}_{\mathbf{R}+\Delta \mathbf{r}+\mathbf{c}}\right)\right]\right\rangle_{\Delta \mathbf{r}}\right\}\right\rangle_{\mathbf{R} \mid \boldsymbol{\Lambda}}\right)\right\rangle_{\mathbf{c}}\right]
\end{aligned}
$$

The derivations are similar to those in the clustered lumpy background case.

\subsection{FOM Derivation}

The characteristic function for a random variable suffices to describe the random variable completely. Its behavior at the origin defines all the moments of the random variable. Similarly, the characteristic functional for a stochastic process offers an alternative to the probability density functional for describing the stochastic process. The mean process function and the covariance function of the process are obtained by taking first- and second-order derivatives of the characteristic functional at the origin as well. These two functions are in the figure of merit, or the SNR in particular, for evaluating the Hotelling observers performance on detection tasks.

\subsubsection{Fréchet derivative}

The concept of the derivative of a functional is called Fréchet derivative [9,60]. For a scalar-valued functional, the Fréchet derivative is a vector of the same dimensionality as the functional argument, which is infinite-dimensional. The definition is similar to the ordinary gradient of a scalar-valued function; if the function $\mathbf{f}$ is perturbed to $\mathbf{f}+\epsilon \mathbf{u}$, where $\mathbf{u}$ is some function in the Hilbert space, then we can define the Fréchet 
derivative $\delta \Psi(\mathbf{f})$ of the scalar functional $\Psi(\mathbf{f})$ via

$$
\lim _{\epsilon \rightarrow 0} \frac{\Psi(\mathbf{f}+\epsilon \mathbf{u})-\Psi(\mathbf{f})}{\epsilon}=(\mathbf{u}, \delta \Psi(\mathbf{f})) .
$$

The Fréchet derivative of a scalar functional is a function. For an infinite-dimensionalvalued functional $\Psi(\mathbf{f})$, i.e., an operator maps a function $\mathbf{f}$ to another function, the Fréchet derivative can be similarly defined as

$$
\lim _{\epsilon \rightarrow 0} \frac{\Psi(\mathbf{f}+\epsilon \mathbf{u})-\Psi(\mathbf{f})}{\epsilon}=(\mathbf{u}, \delta \Psi(\mathbf{f}))=[\delta \Psi(\mathbf{f})]\left(\mathbf{u}^{*}\right) .
$$

Because the result of the limit is infinite dimensional, the inner product should have the same dimensionality, which indicates the Fréchet derivative of an infinitedimensional-valued functional is an operator. The scalar-valued functional maps a function to a scalar, and its first-order Fréchet derivative maps a function to another function. The second-order Fréchet derivative therefore maps a function to an operator since the first-order Fréchet derivative is in fact an operator.

Directly applying the definitions, we can calculate the Fréchet derivative of the characteristic functional of a stochastic process. If $\Psi_{\mathbf{f}}(\boldsymbol{\xi})$ is the CFl for a stochastic process $\mathbf{f}$, the first-order Fréchet derivative of the CFl is $\delta \Psi_{\mathbf{f}}(\boldsymbol{\xi})$. From the definition, we have

$$
\begin{aligned}
\left(\mathbf{u}, \delta \Psi_{\mathbf{f}}(\boldsymbol{\xi})\right) & =\left.\frac{\mathrm{d}}{\mathrm{d} \epsilon} \Psi_{\mathbf{f}}(\boldsymbol{\xi}+\epsilon \mathbf{u})\right|_{\epsilon=0} \\
& =\left.\frac{\mathrm{d}}{\mathrm{d} \epsilon}\left\langle\exp \left[-2 \pi i(\boldsymbol{\xi}+\epsilon \mathbf{u})^{\dagger} \mathbf{f}\right]\right\rangle_{\mathbf{f}}\right|_{\epsilon=0} \\
& =\left\langle\left.\exp \left(-2 \pi i \boldsymbol{\xi}^{\dagger} \mathbf{f}\right) \frac{\mathrm{d}}{\mathrm{d} \epsilon} \exp \left(-2 \pi i \epsilon \mathbf{u}^{\dagger} \mathbf{f}\right)\right|_{\epsilon=0}\right\rangle_{\mathbf{f}} \\
& =\left\langle\left.\exp \left(-2 \pi i \boldsymbol{\xi}^{\dagger} \mathbf{f}\right)\left(-2 \pi i \mathbf{u}^{\dagger} \mathbf{f}\right) \exp \left(-2 \pi i \epsilon \mathbf{u}^{\dagger} \mathbf{f}\right)\right|_{\epsilon=0}\right\rangle_{\mathbf{f}} \\
& =-\left.2 \pi i \mathbf{u}^{\dagger}\left\langle\mathbf{f} \exp \left[-2 \pi i(\boldsymbol{\xi}+\epsilon \mathbf{u})^{\dagger} \mathbf{f}\right]\right\rangle_{\mathbf{f}}\right|_{\epsilon=0} \\
& =-2 \pi i \mathbf{u}^{\dagger}\left\langle\mathbf{f} \exp \left(-2 \pi i \boldsymbol{\xi}^{\dagger} \mathbf{f}\right)\right\rangle_{\mathbf{f}} \\
& =\left(\mathbf{u},-2 \pi i\left\langle\mathbf{f} \exp \left(-2 \pi i \boldsymbol{\xi}^{\dagger} \mathbf{f}\right)\right\rangle_{\mathbf{f}}\right)
\end{aligned}
$$

Because the test function $\mathbf{u}$ is arbitrary, the first-order Fréchet derivative of the CFl for $f$ is

$$
\delta \Psi_{\mathbf{f}}(\boldsymbol{\xi})=-2 \pi i\left\langle\mathbf{f} \exp \left(-2 \pi i \boldsymbol{\xi}^{\dagger} \mathbf{f}\right)\right\rangle_{\mathbf{f}}
$$


The mean process is derived from the first-order Fréchet derivative at the origin,

$$
\langle\mathbf{f}\rangle=(-2 \pi i)^{-1} \boldsymbol{\delta} \Psi_{\mathbf{f}}(0) .
$$

Taking the Fréchet derivative a second time, we can have the second-order Fréchet derivative of the CFl. Noting that both the process and the argument function are real, we have

$$
\begin{aligned}
{\left[\delta^{2} \Psi_{\mathbf{f}}(\boldsymbol{\xi})\right](\mathbf{u}) } & =\left.\frac{\mathrm{d}}{\mathrm{d} \epsilon} \delta \Psi_{\mathbf{f}}(\boldsymbol{\xi}+\epsilon \mathbf{u})\right|_{\epsilon=0} \\
& =\left.\frac{\mathrm{d}}{\mathrm{d} \epsilon}\left\{-2 \pi i\left\langle\mathbf{f} \exp \left[-2 \pi i(\boldsymbol{\xi}+\epsilon \mathbf{u})^{\dagger} \mathbf{f}\right]\right\rangle_{\mathbf{f}}\right\}\right|_{\epsilon=0} \\
& =-2 \pi i\left\langle\mathbf{f} \frac{\mathrm{d}}{\mathrm{d} \epsilon} \exp \left[-\left.2 \pi i\left(\boldsymbol{\xi}^{\dagger} \mathbf{f}+\epsilon \mathbf{u}^{\dagger} \mathbf{f}\right)\right|_{\epsilon=0}\right]\right\rangle_{\mathbf{f}} \\
& =(-2 \pi i)^{2}\left\langle\left.\mathbf{f}\left(\mathbf{u}^{\dagger} \mathbf{f}\right) \exp \left[-2 \pi i(\boldsymbol{\xi}+\epsilon \mathbf{u})^{\dagger} \mathbf{f}\right]\right|_{\epsilon=0}\right\rangle_{\mathbf{f}} \\
& =(-2 \pi i)^{2}\left\langle\mathbf{f}\left(\mathbf{u}^{\dagger} \mathbf{f}\right) \exp \left[-2 \pi i \boldsymbol{\xi}^{\dagger} \mathbf{f}\right]\right\rangle_{\mathbf{f}} \\
& =(-2 \pi i)^{2}\left\langle\mathbf{f}\left(\mathbf{f}^{\dagger} \mathbf{u}\right) \exp \left[-2 \pi i \boldsymbol{\xi}^{\dagger} \mathbf{f}\right]\right\rangle_{\mathbf{f}} \\
& =\left[(-2 \pi i)^{2}\left\langle\exp \left(-2 \pi i \boldsymbol{\xi}^{\dagger} \mathbf{f}\right)\left(\mathbf{f} \mathbf{f}^{\dagger}\right)\right\rangle_{\mathbf{f}}\right] \mathbf{u} .
\end{aligned}
$$

The second order Fréchet derivative of the $\mathrm{CFl}$ is

$$
\delta^{2} \Psi_{\mathbf{f}}(\boldsymbol{\xi})=(-2 \pi i)^{2}\left\langle\mathbf{f} \mathbf{f}^{\dagger} \exp \left[-2 \pi i \boldsymbol{\xi}^{\dagger} \mathbf{f}\right]\right\rangle_{\mathbf{f}}
$$

From its value at the origin, we get the autocorrelation operator of the process,

$$
\left\langle\mathbf{f} \mathbf{f}^{\dagger}\right\rangle=(-2 \pi i)^{-2} \boldsymbol{\delta}^{2} \Psi_{\mathbf{f}}(0) .
$$

The kernel of the autocorrelation operator is commonly called the autocorrelation function.

In fact, all the moments can be derived from successive Fréchet derivatives of the CFl. We demonstrate only the first two orders since only they appear in the SNR of the Hotelling observers on detection tasks. 


\subsubsection{Calculations of clustered lumpy backgrounds}

Given the analytic forms of the CFl for clustered lumpy backgrounds in section 5.3, we can calculate the mean background and the covariance function of the background using Fréchet derivatives.

The CFl for the clustered lumpy background is

$$
\begin{aligned}
& \Psi_{\text {clumpy }}(\boldsymbol{\xi}) \\
= & \mathrm{M}_{N}\left(\left\langle\mathrm{M}_{K}\left(\Psi_{\Lambda}\left(\boldsymbol{\xi} \circ \mathcal{S}_{\mathbf{c}} \circ \mathcal{R}_{-\phi}\right)\right)\right\rangle_{\mathbf{c}, \phi}\right) \\
= & \exp \left(-\bar{N}\left\{1-\left\langle\operatorname { e x p } \left[-\bar{K}\left(1-\left\langle\exp \left\{-2 \pi i \int \mathrm{d} \mathbf{r} \xi(\mathbf{r}) \Lambda\left[\left[R_{\phi}\right](\mathbf{r}-\mathbf{c}-\Delta)\right]\right\}\right.\right.\right.\right.\right.\right. \\
& \rangle_{\Delta}\right)\right]\right\rangle_{\mathbf{c}, \phi}\right\}\right) .
\end{aligned}
$$

Taking the derivative of the functional $\Psi_{\text {clumpy }}(\boldsymbol{\xi}+\epsilon \mathbf{u})$ with respect to $\epsilon$ and calculating it at the origin, we can derive the expression according to the chain rule of the derivative as

$$
\begin{aligned}
\left.\frac{\mathrm{d}}{\mathrm{d} \epsilon} \Psi_{c l u m p y}(\boldsymbol{\xi}+\epsilon \mathbf{u})\right|_{\epsilon=0}= & \mathrm{M}_{N}^{\prime}\left\{\left\langle\mathrm{M}_{K}\left[\Psi_{\boldsymbol{\Lambda}}\left(\boldsymbol{\xi} \circ \mathcal{S}_{\mathbf{c}} \circ \mathcal{R}_{-\phi}\right)\right]\right\rangle_{\mathbf{c}, \phi}\right\} \\
& \cdot\left\langle\left.\mathrm{M}_{K}^{\prime}\left[\Psi_{\Lambda}\left(\boldsymbol{\xi} \circ \mathcal{S}_{\mathbf{c}} \circ \mathcal{R}_{-\phi}\right)\right] \frac{\mathrm{d}}{\mathrm{d} \epsilon} \Psi_{\boldsymbol{\Lambda}}\left[(\boldsymbol{\xi}+\epsilon \mathbf{u}) \circ \mathcal{S}_{\mathbf{c}} \circ \mathcal{R}_{-\phi}\right]\right|_{\epsilon=0}\right\rangle_{\mathbf{c}, \phi}
\end{aligned}
$$

The first-order Fréchet derivative of the CFl for clustered lumpy backgrounds is

$$
\begin{aligned}
\delta \Psi_{\text {clumpy }}(\xi)= & \mathrm{M}_{N}^{\prime}\left\{\left\langle\mathrm{M}_{K}\left[\Psi_{\Lambda}\left(\xi \circ \mathcal{S}_{\mathbf{c}} \circ \mathcal{R}_{-\phi}\right)\right]\right\rangle_{\mathbf{c}, \phi}\right\} \\
& \cdot\left\langle\mathrm{M}_{K}^{\prime}\left[\Psi_{\Lambda}\left(\xi \circ \mathcal{S}_{\mathbf{c}} \circ \mathcal{R}_{-\phi}\right)\right] \delta \Psi_{\Lambda}\left(\boldsymbol{\xi} \circ \mathcal{S}_{\mathbf{c}} \circ \mathcal{R}_{-\phi}\right)\right\rangle_{\mathbf{c}, \phi}
\end{aligned}
$$

The calculation reduces to the Fréchet derivative of the CFl for a single lump $\Lambda$ centered at the position $c$ and oriented at the angle $\phi$. We see

$$
\begin{aligned}
\left.\frac{\mathrm{d}}{\mathrm{d} \epsilon} \Psi_{\Lambda}(\boldsymbol{\xi}+\epsilon \mathbf{u})\right|_{\epsilon=0} & =\left\langle\left.\frac{\mathrm{d}}{\mathrm{d} \epsilon} \exp \left\{-2 \pi i \int \mathrm{d} \mathbf{r}[\xi(\mathbf{r})+\epsilon u(\mathbf{r})] \Lambda\left[\left[R_{\phi}\right](\mathbf{r}-\mathbf{c}-\Delta)\right]\right\}\right|_{\boldsymbol{\epsilon}=0}\right\rangle_{\Delta} \\
& =-2 \pi i\left\langle\left[\mathbf{u}^{\dagger}\left(\boldsymbol{\Lambda} \circ \mathcal{R}_{\phi} \circ \mathcal{S}_{-\mathbf{c}-\Delta}\right)\right] \exp \left[-2 \pi i \boldsymbol{\xi}^{\dagger}\left(\boldsymbol{\Lambda} \circ \mathcal{R}_{\phi} \circ \mathcal{S}_{-\mathbf{c}-\Delta}\right)\right]\right\rangle_{\Delta}
\end{aligned}
$$


The first-order Fréchet derivative of the CFl for a single lump is

$$
\begin{aligned}
& \delta \Psi_{\Lambda}\left(\boldsymbol{\xi} \circ \mathcal{S}_{\mathbf{c}} \circ \mathcal{R}_{-\phi}\right) \\
= & -2 \pi i\left\langle\Lambda\left[\left[R_{\phi}\right](\mathbf{r}-\mathbf{c}-\Delta)\right] \exp \left\{-2 \pi i \int \mathrm{d} \mathbf{r} \xi(\mathbf{r}) \Lambda\left[\left[R_{\phi}\right](\mathbf{r}-\mathbf{c}-\Delta)\right]\right\}\right\rangle_{\Delta} \\
= & -2 \pi i\left\langle\left(\Lambda \circ \mathcal{R}_{\phi} \circ \mathcal{S}_{-\mathbf{c}-\Delta}\right) \exp \left[-2 \pi i \boldsymbol{\xi}^{\dagger}\left(\Lambda \circ \mathcal{R}_{\phi} \circ \mathcal{S}_{-\mathbf{c}-\Delta}\right)\right]\right\rangle_{\Delta} .
\end{aligned}
$$

When substituting this expression into the equation (5.5.11), we can get the final form of the first-order Fréchet derivative of the CFl for the background.

$\mathrm{A} \mathrm{CFl} \mathrm{equals} \mathrm{unity} \mathrm{at} \mathrm{the} \mathrm{origin} \mathrm{while} \mathrm{the} \mathrm{probability-generating} \mathrm{function} \mathrm{of} \mathrm{any}$ non-negative discrete random variable is unity at 1 . The first-order derivative of a probability-generating function evaluated at 1 is the mean of the random variable. Based on these results, the mean background is

$$
\begin{aligned}
\langle\mathrm{b}\rangle_{\text {clumpy }} & =\bar{N}\left\langle\bar{K}\left\langle\Lambda \circ \mathcal{R}_{\phi} \circ \mathcal{S}_{-\mathbf{c}-\Delta}\right\rangle_{\Delta}\right\rangle_{\mathbf{c}, \phi} \\
& =\bar{N} \bar{K}\left\langle\Lambda\left[\left[R_{\phi}\right](\mathbf{r}-\mathbf{c}-\Delta)\right]\right\rangle_{\Delta, \mathbf{c}, \phi} .
\end{aligned}
$$

Here we employ the result about the first-order Fréchet derivative of the CFl for a single lump.

To calculate the second-order Fréchet derivative of the CFl for the whole background, we need to evaluate the derivative of $\delta \Psi_{\text {clumpy }}(\boldsymbol{\xi}+\epsilon \mathbf{u})$ with respect to $\epsilon$ at the origin. From the equation (5.5.11), we know

$$
\begin{aligned}
& \left.\frac{\mathrm{d}}{\mathrm{d} \epsilon} \delta \Psi_{\text {clumpy }}(\xi+\epsilon \mathrm{u})\right|_{\epsilon=0} \\
= & \left.\frac{\mathrm{d}}{\mathrm{d} \epsilon} \mathrm{M}_{N}^{\prime}\left(\left\langle\mathrm{M}_{K}\left\{\Psi_{\Lambda}\left[(\xi+\epsilon \mathbf{u}) \circ \mathcal{S}_{\mathbf{c}} \circ \mathcal{R}_{-\phi}\right]\right\}\right\rangle_{\mathbf{c}, \phi}\right)\right|_{\epsilon=0} \\
& \cdot\left\langle\mathrm{M}_{K}^{\prime}\left(\Psi_{\Lambda}\left(\xi \circ \mathcal{S}_{\mathbf{c}} \circ \mathcal{R}_{-\phi}\right)\right) \delta \Psi_{\Lambda}\left(\xi \circ \mathcal{S}_{\mathbf{c}} \circ \mathcal{R}_{-\phi}\right)\right\rangle_{\mathbf{c}, \phi} \\
+ & \mathrm{M}_{N}^{\prime}\left\{\left\langle\mathrm{M}_{K}\left[\Psi_{\Lambda}\left(\xi \circ \mathcal{S}_{\mathbf{c}} \circ \mathcal{R}_{-\phi}\right)\right]\right\rangle_{\mathbf{c}, \phi}\right\} \\
& \cdot\left\langle\left.\frac{\mathrm{d}}{\mathrm{d} \epsilon} \mathrm{M}_{K}^{\prime}\left\{\Psi_{\Lambda}\left[(\xi+\epsilon \mathbf{u}) \circ \mathcal{S}_{\mathbf{c}} \circ \mathcal{R}_{-\phi}\right]\right\}\right|_{\epsilon=0} \delta \Psi_{\Lambda}\left(\xi \circ \mathcal{S}_{\mathbf{c}} \circ \mathcal{R}_{-\phi}\right)\right\rangle_{\mathbf{c}, \phi} \\
+ & \mathrm{M}_{N}^{\prime}\left\{\left\langle\mathrm{M}_{K}\left[\Psi_{\Lambda}\left(\xi \circ \mathcal{S}_{\mathbf{c}} \circ \mathcal{R}_{-\phi}\right)\right]\right\rangle_{\mathbf{c}, \phi}\right\} \\
& \cdot\left\langle\left.\mathrm{M}_{K}^{\prime}\left[\Psi_{\Lambda}\left(\xi \circ \mathcal{S}_{\mathbf{c}} \circ \mathcal{R}_{-\phi}\right)\right] \frac{\mathrm{d}}{\mathrm{d} \epsilon} \delta \Psi_{\Lambda}\left[(\boldsymbol{\xi}+\epsilon \mathbf{u}) \circ \mathcal{S}_{\mathbf{c}} \circ \mathcal{R}_{-\phi}\right]\right|_{\epsilon=0}\right\rangle_{\mathbf{c}, \phi} .
\end{aligned}
$$

The first term in the right-hand side of the equation, denoted with the superscript 1 , 
results in

$$
\begin{aligned}
\left.\frac{\mathrm{d}}{\mathrm{d} \epsilon} \delta \Psi_{\text {clumpy }}^{(1)}(\boldsymbol{\xi}+\epsilon \mathbf{u})\right|_{\epsilon=0}= & \mathrm{M}_{N}^{\prime \prime}\left\{\left\langle\mathrm{M}_{K}\left[\Psi_{\boldsymbol{\Lambda}}\left(\boldsymbol{\xi} \circ \mathcal{S}_{\mathbf{c}} \circ \mathcal{R}_{-\phi}\right)\right]\right\rangle_{\mathbf{c}, \phi}\right\} \\
& \cdot\left\langle\mathrm{M}_{K}^{\prime}\left[\Psi_{\boldsymbol{\Lambda}}\left(\boldsymbol{\xi} \circ \mathcal{S}_{\mathbf{c}} \circ \mathcal{R}_{-\phi}\right)\right] \delta \Psi_{\Lambda}^{\dagger}\left(\boldsymbol{\xi} \circ \mathcal{S}_{\mathbf{c}} \circ \mathcal{R}_{-\phi}\right) \mathbf{u}\right\rangle_{\mathbf{c}, \phi} \\
& \cdot\left\langle\mathrm{M}_{K}^{\prime}\left[\Psi_{\Lambda}\left(\xi \circ \mathcal{S}_{\mathbf{c}} \circ \mathcal{R}_{-\phi}\right)\right] \delta \Psi_{\Lambda}\left(\xi \circ \mathcal{S}_{\mathbf{c}} \circ \mathcal{R}_{-\phi}\right)\right\rangle_{\mathbf{c}, \phi},
\end{aligned}
$$

where the operator $\delta \Psi_{\Lambda}^{\dagger}$ is defined as

$$
\delta \Psi_{\Lambda}^{\dagger}\left(\boldsymbol{\xi} \circ \mathcal{S}_{\mathbf{c}} \circ \mathcal{R}_{-\phi}\right)=-2 \pi i\left\langle\left(\Lambda \circ \mathcal{R}_{\phi} \circ \mathcal{S}_{-\mathbf{c}-\Delta}\right)^{\dagger} \exp \left[-2 \pi i \boldsymbol{\xi}^{\dagger}\left(\boldsymbol{\Lambda} \circ \mathcal{R}_{\phi} \circ \mathcal{S}_{-\mathbf{c}-\Delta}\right)\right]\right\rangle_{\Delta} .
$$

The first term of the second-order Fréchet derivative of the CFl for the background is thus

$$
\begin{aligned}
\delta^{2} \Psi_{\text {clumpy }}^{(1)}(\boldsymbol{\xi})= & \mathrm{M}_{N}^{\prime}\left\{\left\langle\mathrm{M}_{K}\left[\Psi_{\Lambda}\left(\boldsymbol{\xi} \circ \mathcal{S}_{\mathbf{c}} \circ \mathcal{R}_{-\phi}\right)\right]\right\rangle_{\mathbf{c}, \phi}\right\} \\
& \cdot\left\langle\mathrm{M}_{K}^{\prime}\left[\Psi_{\Lambda}\left(\xi \circ \mathcal{S}_{\mathbf{c}} \circ \mathcal{R}_{-\phi}\right)\right] \delta \Psi_{\Lambda}\left(\boldsymbol{\xi} \circ \mathcal{S}_{\mathbf{c}} \circ \mathcal{R}_{-\phi}\right)\right\rangle_{\mathbf{c}, \phi} \\
& \cdot\left\langle\mathrm{M}_{K}^{\prime}\left[\Psi_{\Lambda}\left(\xi \circ \mathcal{S}_{\mathbf{c}} \circ \mathcal{R}_{-\phi}\right)\right] \delta \Psi_{\Lambda}^{\dagger}\left(\boldsymbol{\xi} \circ \mathcal{S}_{\mathbf{c}} \circ \mathcal{R}_{-\phi}\right)\right\rangle_{\mathbf{c}, \phi} .
\end{aligned}
$$

The second-order derivative of the probability-generating function evaluated at 1 is the difference of the second-order moment and the first-order moment,

$$
\mathrm{M}_{N}^{\prime \prime}(1)=\left\langle N^{2}\right\rangle-\langle N\rangle
$$

For a Poisson random variable, the variance is the same as the mean. The above quantity is thus the square of the mean, i.e. $\langle N\rangle^{2}$. From this, the first term of the correlation operator is

$$
\left\langle\mathbf{b b}^{\dagger}\right\rangle_{\text {clumpy }}^{(1)}=\bar{N}^{2} \bar{K}^{2}\left\langle\Lambda \circ \mathcal{R}_{\phi} \circ \mathcal{S}_{-\mathbf{c}-\Delta}\right\rangle_{\Delta, \mathbf{c}, \phi}\left\langle\left(\Lambda \circ \mathcal{R}_{\phi} \circ \mathcal{S}_{-\mathbf{c}-\Delta}\right)^{\dagger}\right\rangle_{\Delta, \mathbf{c}, \phi}
$$

This term is equivalent to the operator $\langle\mathbf{b}\rangle\left\langle\mathbf{b}^{\dagger}\right\rangle$.

The treatment of the first term also applies to the other two terms. The second term in the right-hand side of the equation (5.5.15) is

$$
\begin{aligned}
& \left.\frac{\mathrm{d}}{\mathrm{d} \epsilon} \boldsymbol{\delta} \Psi_{\text {clumpy }}^{(2)}(\boldsymbol{\xi}+\epsilon \mathbf{u})\right|_{\epsilon=0} \\
= & \mathrm{M}_{N}^{\prime}\left\{\left\langle\mathrm{M}_{K}\left[\Psi_{\Lambda}\left(\boldsymbol{\xi} \circ \mathcal{S}_{\mathbf{c}} \circ \mathcal{R}_{-\phi}\right)\right]\right\rangle_{\mathbf{c}, \phi}\right\} \\
& \cdot\left\langle\mathrm{M}_{K}^{\prime \prime}\left[\Psi_{\boldsymbol{\Lambda}}\left(\boldsymbol{\xi} \circ \mathcal{S}_{\mathbf{c}} \circ \mathcal{R}_{-\phi}\right)\right] \delta \Psi_{\Lambda}^{\dagger}\left(\boldsymbol{\xi} \circ \mathcal{S}_{\mathbf{c}} \circ \mathcal{R}_{-\phi}\right) \mathbf{u} \delta \Psi_{\Lambda}\left(\boldsymbol{\xi} \circ \mathcal{S}_{\mathbf{c}} \circ \mathcal{R}_{-\phi}\right)\right\rangle_{\mathbf{c}, \phi}
\end{aligned}
$$


We can find the second term of the second-order Fréchet derivative of the CFl for the background as

$$
\begin{aligned}
\delta^{2} \Psi_{\text {clumpy }}^{(2)}(\boldsymbol{\xi})= & \mathrm{M}_{N}^{\prime}\left\{\left\langle\mathrm{M}_{K}\left[\Psi_{\Lambda}\left(\xi \circ \mathcal{S}_{\mathrm{c}} \circ \mathcal{R}_{-\phi}\right)\right]\right\rangle_{\mathbf{c}, \phi}\right\}\left\langle\mathrm{M}_{K}^{\prime \prime}\left[\Psi_{\Lambda}\left(\boldsymbol{\xi} \circ \mathcal{S}_{\mathbf{c}} \circ \mathcal{R}_{-\phi}\right)\right]\right. \\
& \left.\cdot \delta \Psi_{\Lambda}\left(\xi \circ \mathcal{S}_{\mathbf{c}} \circ \mathcal{R}_{-\phi}\right) \delta \Psi_{\Lambda}^{\dagger}\left(\xi \circ \mathcal{S}_{\mathbf{c}} \circ \mathcal{R}_{-\phi}\right)\right\rangle_{\mathrm{c}, \phi} .
\end{aligned}
$$

After evaluating the second term of the Fréchet derivative at the origin, we can derive the second term of the correlation operator of the background. It is

$$
\left\langle\mathrm{bb}^{\dagger}\right\rangle_{\text {clumpy }}^{(2)}=\overline{N K}^{2}\left\langle\left\langle\Lambda \circ \mathcal{R}_{\phi} \circ \mathcal{S}_{-\mathrm{c}-\Delta}\right\rangle_{\Delta}\left\langle\left(\Lambda \circ \mathcal{R}_{\phi} \circ \mathcal{S}_{-\mathrm{c}-\Delta}\right)^{\dagger}\right\rangle_{\Delta}\right\rangle_{\mathrm{c}, \phi}
$$

In this term, the product of the conditional mean lump function and its adjoint is averaged over the cluster center $\mathbf{c}$ and the orientation angle $\phi$ simultaneously. This is intuitively right since $\mathrm{c}$ and $\phi$ are the same for all the lumps inside a single cluster.

The last term in the equation (5.5.15) is calculated as

$$
\begin{aligned}
\left.\frac{\mathrm{d}}{\mathrm{d} \epsilon} \boldsymbol{\delta} \Psi_{\text {clumpy }}^{(3)}(\boldsymbol{\xi}+\epsilon \mathbf{u})\right|_{\epsilon=0}= & \mathrm{M}_{N}^{\prime}\left\{\left\langle\mathrm{M}_{K}\left[\Psi_{\Lambda}\left(\boldsymbol{\xi} \circ \mathcal{S}_{\mathbf{c}} \circ \mathcal{R}_{-\phi}\right)\right]\right\rangle_{\mathbf{c}, \phi}\right\} \\
& \cdot\left\langle\mathrm{M}_{K}^{\prime}\left[\Psi_{\boldsymbol{\Lambda}}\left(\boldsymbol{\xi} \circ \mathcal{S}_{\mathbf{c}} \circ \mathcal{R}_{-\phi}\right)\right]\left[\boldsymbol{\delta}^{2} \Psi_{\boldsymbol{\Lambda}}\left(\boldsymbol{\xi} \circ \mathcal{S}_{\mathbf{c}} \circ \mathcal{R}_{-\phi}\right)\right](\mathbf{u})\right\rangle_{\mathbf{c}, \phi}
\end{aligned}
$$

The last term of the Fréchet derivative of the CFl for the background is therefore

$$
\begin{aligned}
\delta^{2} \Psi_{\text {clumpy }}^{(3)}(\boldsymbol{\xi})= & \mathrm{M}_{N}^{\prime}\left\{\left\langle\mathrm{M}_{K}\left[\Psi_{\boldsymbol{\Lambda}}\left(\boldsymbol{\xi} \circ \mathcal{S}_{\mathbf{c}} \circ \mathcal{R}_{-\phi}\right)\right]\right\rangle_{\mathbf{c}, \phi}\right\} \\
& \cdot\left\langle\mathrm{M}_{K}^{\prime}\left[\Psi_{\boldsymbol{\Lambda}}\left(\boldsymbol{\xi} \circ \mathcal{S}_{\mathbf{c}} \circ \mathcal{R}_{-\phi}\right)\right] \delta^{2} \Psi_{\boldsymbol{\Lambda}}\left(\boldsymbol{\xi} \circ \mathcal{S}_{\mathbf{c}} \circ \mathcal{R}_{-\phi}\right)\right\rangle_{\mathbf{c}, \phi} .
\end{aligned}
$$

To find out the second-order Fréchet derivative of the CFl for a single lump $\delta^{2} \Psi_{\Lambda}\left(\xi \circ \mathcal{S}_{\mathbf{c}} \circ \mathcal{R}_{-\phi}\right)$, we employ the definition of the Fréchet derivative in equation (5.5.13),

$$
\begin{aligned}
& {\left[\boldsymbol{\delta}^{2} \Psi_{\boldsymbol{\Lambda}}\left(\boldsymbol{\xi} \circ \mathcal{S}_{\mathbf{c}} \circ \mathcal{R}_{-\phi}\right)\right](\mathbf{u}) } \\
= & \left.\frac{\mathrm{d}}{\mathrm{d} \epsilon}\left\{-2 \pi i\left\langle\left(\boldsymbol{\Lambda} \circ \mathcal{R}_{\phi} \circ \mathcal{S}_{-\mathbf{c}-\Delta}\right) \exp \left[-2 \pi i(\boldsymbol{\xi}+\epsilon \mathbf{u})^{\dagger}\left(\boldsymbol{\Lambda} \circ \mathcal{R}_{\phi} \circ \mathcal{S}_{-\mathbf{c}-\Delta}\right)\right]\right\rangle_{\Delta}\right\}\right|_{\epsilon=0} \\
= & (-2 \pi i)^{2}\left\langle\left(\boldsymbol{\Lambda} \circ \mathcal{R}_{\phi} \circ \mathcal{S}_{-\mathbf{c}-\Delta}\right)\left(\Lambda \circ \mathcal{R}_{\phi} \circ \mathcal{S}_{-\mathbf{c}-\Delta}\right)^{\dagger} \exp \left[-2 \pi i \boldsymbol{\xi}^{\dagger}\left(\Lambda \circ \mathcal{R}_{\phi} \circ \mathcal{S}_{-\mathbf{c}-\Delta}\right)\right]\right\rangle_{\Delta} \mathbf{u}
\end{aligned}
$$

By substituting this expression into the last term and taking $\boldsymbol{\xi}$ as zero, we can get the last term of the correlation operator of the background,

$$
\left\langle\mathbf{b b}^{\dagger}\right\rangle_{\text {clumpy }}^{(3)}=\overline{N K}\left\langle\left(\Lambda \circ \mathcal{R}_{\phi} \circ \mathcal{S}_{-\mathbf{c}-\Delta}\right)\left(\Lambda \circ \mathcal{R}_{\phi} \circ \mathcal{S}_{-\mathbf{c}-\Delta}\right)^{\dagger}\right\rangle_{\Delta, \mathbf{c}, \phi}
$$


In this term, the product of the lump function and its adjoint is averaged over all the random variables simultaneously.

Now we are ready to write out the complete correlation operator of clustered lumpy backgrounds,

$$
\begin{aligned}
\left\langle\mathbf{b b}^{\dagger}\right\rangle_{\text {clumpy }} & =\bar{N}^{2} \bar{K}^{2}\left\langle\Lambda \circ \mathcal{R}_{\phi} \circ \mathcal{S}_{-\mathbf{c}-\Delta}\right\rangle_{\Delta, \mathbf{c}, \phi}\left\langle\left(\Lambda \circ \mathcal{R}_{\phi} \circ \mathcal{S}_{-\mathbf{c}-\Delta}\right)^{\dagger}\right\rangle_{\Delta, \mathbf{c}, \phi} \\
& +\bar{N} \bar{K}^{2}\left\langle\left\langle\Lambda \circ \mathcal{R}_{\phi} \circ \mathcal{S}_{-\mathbf{c}-\Delta}\right\rangle_{\Delta}\left\langle\left(\Lambda \circ \mathcal{R}_{\phi} \circ \mathcal{S}_{-\mathbf{c}-\Delta}\right)^{\dagger}\right\rangle_{\Delta}\right\rangle_{\mathbf{c}, \phi} \\
& +\bar{N} \bar{K}\left\langle\left(\Lambda \circ \mathcal{R}_{\phi} \circ \mathcal{S}_{-\mathbf{c}-\Delta}\right)\left(\Lambda \circ \mathcal{R}_{\phi} \circ \mathcal{S}_{-\mathbf{c}-\Delta}\right)^{\dagger}\right\rangle_{\Delta, \mathbf{c}, \phi}
\end{aligned}
$$

Because the first term is the same as the operator $\langle b\rangle\left\langle\mathbf{b}^{\ddagger}\right\rangle$, the covariance operator is the sum of the remaining two terms,

$$
\begin{aligned}
\mathcal{K}_{\text {clumpy }} & =\left\langle\mathrm{bb}^{\dagger}\right\rangle_{\text {clumpy }}-\langle\mathrm{b}\rangle\left\langle\mathrm{b}^{\dagger}\right\rangle \\
& =\bar{N} \bar{K}^{2}\left\langle\left\langle\Lambda \circ \mathcal{R}_{\phi} \circ \mathcal{S}_{-\mathbf{c}-\Delta}\right\rangle_{\Delta}\left\langle\left(\Lambda \circ \mathcal{R}_{\phi} \circ \mathcal{S}_{-\mathbf{c}-\Delta}\right)^{\dagger}\right\rangle_{\Delta}\right\rangle_{\mathbf{c}, \phi} \\
& +\bar{N} \bar{K}\left\langle\left(\Lambda \circ \mathcal{R}_{\phi} \circ \mathcal{S}_{-\mathbf{c}-\Delta}\right)\left(\Lambda \circ \mathcal{R}_{\phi} \circ \mathcal{S}_{-\mathbf{c}-\Delta}\right)^{\dagger}\right\rangle_{\Delta, \mathbf{c}, \phi} \cdot
\end{aligned}
$$

From this operator, we can find the covariance function as

$$
\begin{aligned}
K\left(\mathbf{r}, \mathbf{r}^{\prime}\right) & =\bar{N} \bar{K}^{2}\left\langle\left\langle\Lambda\left[\left[R_{\phi}\right](\mathbf{r}-\mathbf{c}-\Delta)\right]\right\rangle_{\Delta}\left\langle\Lambda\left[\left[R_{\phi}\right]\left(\mathbf{r}^{\prime}-\mathbf{c}-\Delta^{\prime}\right)\right]\right\rangle_{\Delta}^{\prime}\right\rangle_{\mathbf{c}, \phi} \\
& +\bar{N} \bar{K}\left\langle\Lambda\left[\left[R_{\phi}\right](\mathbf{r}-\mathbf{c}-\Delta)\right] \Lambda\left[\left[R_{\phi}\right]\left(\mathbf{r}^{\prime}-\mathbf{c}-\Delta\right)\right]\right\rangle_{\Delta, \mathbf{c}, \phi} .
\end{aligned}
$$

This result is exactly the same as the one Bochud has got in his paper [14], although he derived it directly from the probability density functions. By means of the characteristic functionals, we can have all the moments in a systematic way.

With the CFls in the last section, we can surely derive the mean process and the covariance function for the visible image in our model imaging system and the SNR of Hotelling observers as well. The results should be the same. The procedure is demonstrated above, although the detailed derivations are omitted. 


\section{Chapter 6}

\section{Numerical Simulations}

To design lenses using objective image qualities as merit functions, we need to compute numerically the SNR developed in the equations (4.3.12), (4.3.17) and (4.3.18). ${ }^{1}$ We should not get lost in those messy expressions. It is interesting to see that all the information about the system is encapsulated in two functions, the total point response function $\mathrm{p}_{t o t}\left(\mathrm{r}^{\prime}, \mathbf{R} \mid z\right)$ and the factor $\mathrm{Q}(\mathbf{R}, z)$ describing the fluorescent screen. The object randomness is self-contained in its mean function $\bar{b}$ and covariance function $K_{b}$. We can therefore treat the object statistics separately from the system design.

The object here is composed of the signal to be detected superimposed on the additive background in signal-present situation, and only the background in signalabsent situation. In this work, we will investigate the task in the signal-known-exactly (SKE) and background-known-statistically (BKS) case. The signal profile and the background statistics are discussed in section 6.1.

When designing the system, we only need to consider the two functions $\mathrm{p}_{\text {tot }}$ and Q. The function $\mathrm{Q}(\mathbf{R}, z)$ is constant by definition when the the total number of visible light photons generated by an absorbed x-ray photon $m$ is independent of the absorption depth $z$ and the position $\mathbf{R}$. The independence is reasonable for well-manufactured mammographic screens. We therefore assume $\mathrm{Q}$ as constant in our simulation. Only the other function, the depth-dependent total point response function $\mathrm{p}_{t o t}\left(\mathbf{r}^{\prime}, \mathbf{R} \mid z\right)$, needs careful analysis.

The depth-dependent total point response function $\mathrm{p}_{t o t}\left(\mathbf{r}^{\prime}, \mathbf{R} \mid z\right)$ appears in both the mean image vector and the image covariance matrix. The $i^{\text {th }}$ element of the mean image vector is the integral $\iint \mathrm{d}^{2} r^{\prime} \mathrm{p}_{t o t}\left(\mathbf{r}^{\prime}, \mathbf{R} \mid z\right) h_{i}\left(\mathbf{r}^{\prime}\right)$. Since pixels in the CCD do not

\footnotetext{
${ }^{1}$ This chapter is extracted from a paper accepted by JOSA A on July 29, 2004. The chapter can be read more or less independently.
} 
overlap, the first term of the covariance matrix is a diagonal matrix with the elements $\mathcal{D} \mathcal{H}_{1} f$, the same as the mean image vector. After examining the other two terms of the covariance matrix, we find they both contain the integral $\iint \mathrm{d}^{2} r^{\prime} \mathrm{p}_{t o t}\left(\mathbf{r}^{\prime}, \mathbf{R} \mid z\right) h_{i}\left(\mathbf{r}^{\prime}\right)$. This integral is the basic building block of the numerical SNR computation.

The depth-dependent total point response function $\mathrm{p}_{t o t}\left(\mathbf{r}^{\prime}, \mathbf{R} \mid z\right)$ is defined by three functions: the spatial point spread function of the screen $p_{d}(r, R \mid z)$, the passing probability of a visible photon pass $(\mathbf{r}, \mathbf{R} \mid z)$ at $\mathbf{r}$ with its parent $\mathrm{x}$-ray photon at $\mathbf{R}$, and the point response function of the optical imaging system $\mathrm{p}_{\mathrm{g}}\left(\mathbf{r}^{\prime}, \mathbf{r} \mid \mathbf{R}, z\right)$. By the PDF of the displacement (3.2.27) on the CCD detector plane, the integral can be written as:

$$
\begin{aligned}
& \iint \mathrm{d}^{2} r^{\prime} \mathrm{p}_{t o t}\left(\mathbf{r}^{\prime}, \mathbf{R} \mid z\right) h_{i}\left(\mathbf{r}^{\prime}\right) \\
= & \iint \mathrm{d}^{2} r^{\prime} \iint \mathrm{d}^{2} r \operatorname{p}_{\mathrm{d}}(\mathbf{r}, \mathbf{R} \mid z) \operatorname{pass}(\mathbf{r}, \mathbf{R} \mid z) \mathrm{p}_{\mathrm{g}}\left(\mathbf{r}^{\prime}, \mathbf{r} \mid \mathbf{R}, z\right) \\
= & \iint \mathrm{d}^{2} r \mathrm{p}_{\mathrm{d}}(\mathbf{r}, \mathbf{R} \mid z) \operatorname{pass}(\mathbf{r}, \mathbf{R} \mid z) \iint \mathrm{d}^{2} \Delta r^{\prime} \operatorname{pr}_{\Delta \mathbf{r}}\left(\Delta \mathbf{r}^{\prime} \mid \mathbf{r}, \mathbf{R}, z\right) h_{i}\left(\mathbf{r}+\Delta \mathbf{r}^{\prime}\right) \\
= & \iint \mathrm{d}^{2} r \mathrm{p}_{\mathrm{d}}(\mathbf{r}, \mathbf{R} \mid z) \operatorname{pass}(\mathbf{r}, \mathbf{R} \mid z) \iint_{\Omega(\mathbf{r})} \mathrm{d} \Omega \operatorname{pr}_{\hat{\mathbf{s}}}(\hat{\mathbf{s}} \mid \mathbf{r}, \mathbf{R}, z) h_{i}\left(\mathbf{r}+\Delta \mathbf{r}^{\prime}(\mathbf{r}+\mathbf{R}, \hat{\mathbf{s}})\right) .
\end{aligned}
$$

When referred back to the expressions of $\mathrm{p}_{\mathrm{d}}$, pass and $\mathrm{pr}_{\hat{\mathbf{s}}}$ in (3.2.13), (3.2.19) and (3.2.18), the final integral form used in the Monte Carlo calculation is

$$
\begin{aligned}
\iint \mathrm{d}^{2} r^{\prime} \mathrm{p}_{t o t}\left(\mathbf{r}^{\prime}, \mathbf{R} \mid z\right) h_{i}\left(\mathbf{r}^{\prime}\right)= & \bar{K}(\mathbf{R}, z) \iint \mathrm{d}^{2} r \frac{\int_{2 \pi} \mathrm{d} \Omega^{\prime} \operatorname{BTDF}\left(\mathbf{r}, \hat{\mathbf{s}}^{\prime}, \mathbf{R}, \hat{z} \mid z\right)}{\iint_{\infty} \mathrm{d}^{2} r^{\prime} \int_{2 \pi} \mathrm{d} \Omega^{\prime} \operatorname{BTDF}\left(\mathbf{r}^{\prime}, \hat{\mathbf{s}}^{\prime}, \mathbf{R}, \hat{z} \mid z\right)} \\
& \cdot \int_{\Omega(\mathbf{r})} \mathrm{d} \Omega \frac{\operatorname{BTDF}(\mathbf{r}, \hat{\mathbf{s}}, \mathbf{R}, \hat{z} \mid z)}{\int_{2 \pi} \mathrm{d} \Omega^{\prime} \operatorname{BTDF}\left(\mathbf{r}, \hat{\mathbf{s}}^{\prime}, \mathbf{R}, \hat{z} \mid z\right)} h_{i}\left(\mathbf{r}+\Delta \mathbf{r}^{\prime}(\hat{\mathbf{s}}, \mathbf{r})\right) .
\end{aligned}
$$

The first fraction is the spatial marginal of the BTDF and the second one is the conditional angular part on the given spatial position. The optical system manifests itself in the integration solid angle $\Omega(\mathbf{r})$ and the transformation $\Delta \mathbf{r}^{\prime}(\hat{\mathbf{s}}, \mathbf{r})$. The mean gain of the screen $\bar{K}$ can be independent of the x-ray absorption position $\mathbf{R}$ and the depth $z$. The inner and outer integrals can be computed with Monte Carlo 
applications, where the position vectors $\mathbf{r}$ and the direction vector $\hat{\mathbf{s}}$ are of a visible photon, and can be sampled from the corresponding probability densities. To find the displacement $\Delta \mathbf{r}^{\prime}$ on the CCD, we can employ a geometrical optical ray-tracing routine to trace a ray from $\mathbf{r}$ on the screen propagating in the direction $\hat{\mathbf{s}}$ to CCD. Since we only need $\bar{K}$ and $Q$, the entire PDF of the gain is not necessary.

\subsection{Backgrounds and Signals}

In statistical description of objects, the complete probability density is again not necessary but only the first two moments for the Hotelling observer. In fact, only the mean and the covariance function of the background are needed since the signal is deterministic. There exist two types of backgrounds developed mainly for medical imaging, namely, lumpy backgrounds and clustered lumpy backgrounds. Both of them are stochastic processes characterized by employing random parameters in deterministic function forms, which makes them mathematically tractable.

The lumpy background is proposed to simulate the image variability in nuclear medicine [57]. A sample function of the lumpy background is

$$
f_{\text {lumpy }}(\mathbf{r})=\sum_{n=1}^{N} \Lambda\left(\mathbf{r}-\mathbf{c}_{n} \mid a_{n}, s_{n}\right),
$$

where $\mathbf{r}$ is a spatial variable in the two or three-dimensional object space, $\Lambda(\cdot)$ is the deterministic lump profile function, $N$ is the number of lumps. For the $n^{\text {th }}$ lump, $\mathbf{c}_{n}$ is the center of the lump function, $a_{n}$ is the magnitude of the lump function, and $s_{n}$ is the parameter controlling the lump width. The random parameters consist of $N$ and the set of $\mathbf{c}_{n}, a_{n}$ and $s_{n}$. The number of lumps $N$ is Poisson-distributed with the mean number of $\bar{N}$. Each center $\mathbf{c}_{n}$ is uniformly distributed inside the field of view. All of $a_{n}$ and $s_{n}$ can be deterministic sometimes. The lump profiles are Gaussian as first proposed, but can be other forms as well. With one realization of lumpy backgrounds as the mean process, the Poisson point process simulating noisy images 
is non-stationary, which is more practical than stationary flood images when used in medical imaging. At the same time, the lumpy background is itself stationary. When the lumpy backgrounds with added Poisson noise are used as the simulated images, the ensemble images are stationary as well. This background could be the background model in our study, however, its visual appearance is far from realistic mammographic images.

The clustered lumpy background is a way of generating textures that simulate real mammograms while still being analytically tractable [14]. It is generated by first selecting a random number of cluster centers in the object area. Then each cluster center is replaced by a random number of blob centers according to certain distribution density about the center. At last, there is a blob centered at each blob position. The blobs have similar shape functions different by the parameters. The shape is elliptical with random orientation. This random background is a wide-sensed stationary background with constant mean function and shift-invariant covariance function. A sample function is

$$
f_{\text {clumpy }}(\mathbf{r})=\sum_{n=1}^{N} \sum_{k=1}^{K_{n}} \Lambda\left(\left[R_{\phi_{n}}\right]\left(\mathbf{r}-\mathbf{c}_{n}-\Delta_{n k}\right) \mid \alpha\right),
$$

where $N$ is the number of clusters, $K_{n}$ is the number of lumps in the $n^{\text {th }}$ cluster, $\Delta_{n k}$ is the center of the $k^{\text {th }}$ lump function in the $n^{\text {th }}$ cluster, $\phi_{n}$ is the orientation of the lumps in the $n^{\text {th }}$ cluster, and $\alpha$ contains the parameters characterizing the shape of the lumps. The rotation of the lumps is represented by the rotation matrix $R_{\phi_{n}}$. The angle $\phi_{n}$ is uniformly distributed between 0 and $2 \pi . N$ and $\left\{K_{n}\right\}$ are all Poisson distributed with means $\bar{N}$ and $\bar{K}$, respectively. The center of a cluster $\mathbf{c}_{n}$ is uniformly distributed inside the field of view, and the centers of the lumps within this cluster are Gaussian distributed around $\mathbf{c}_{n}$. That is, $\boldsymbol{\Delta}_{n k}$ is Gaussian distributed around $\mathbf{c}_{n}$ with the width $\sigma_{\Delta}$. Bochud uses a two-dimensional asymmetric lump given in polar 
coordinates as

$$
\Lambda\left(r, \phi \mid \gamma, \beta, l_{x}, l_{y}\right)=\exp \left[-\gamma r^{\beta} \sqrt{\frac{l_{y}^{2} \cos ^{2}(\phi)+l_{x}^{2} \sin ^{2}(\phi)}{l_{x}^{2} l_{y}^{2}}}\right] .
$$

This function is chosen to match the power spectrum of a set of normal mammograms. Two images of clustered lumpy backgrounds are shown in Fig. 6.1.

We use clustered lumpy backgrounds as the background in our simulation because we study a model mammographic system in particular. We follow the distributions chosen in Bochud's work, with the unit amplitude of every lump. The mean process of the clustered lumpy background is a constant $\bar{b}$. The probability density function of the primary positions $\mathbf{R}$ in signal-absent case is therefore a uniform function inside the field of view, and the strength of each sample used in the later Monte Carlo simulation is $\bar{b}$. The covariance function of the background can be found in ref. [14].

The deterministic signal profile is Gaussian. It is additive to the background, making a signal-present object. The probability density function of the primary positions in the signal-present case is largely uniform with a small bump at the location of the signal. The covariance function is the same as that in signal-absent case. The peak amplitude of a additive signal is several times smaller than the mean amplitude of the background since significant signals are so easy to detect that there is little advantage to optimize the imaging system. The center of the signal can be anywhere inside the field of view but known to the observer, which is called signal known exactly, but variable, or $S K E V$ case. When studying the SKEV problem, we can generate a SNR map versus the variable signal location [19].

\subsection{Scattering Model of Screens}

Fluorescent screens absorb x-ray photons and scatter visible light. The generalized BTDFs describe the joint spatial and directional distributions of output visible light after screens. Although we are lack of the knowledge of the full function, parts of the function can be constructed based on the results in wave-scattering research. 
The light scattering through random media is an active and extensive field with abundant literature $[38,68]$. The random media of our interest are called random scatterers, a random distribution of many discrete scatterers. The cross section is a convenient term to describe the scattering characteristics of a discrete object, such as a scatterer $[9,38]$. The total scattering cross section is defined as

$$
\sigma_{s}=\frac{\text { total scattered flux }}{\text { normal irradiance }}
$$

where the term normal irradiance is the power per unit area normally incident on the scatterer interface. The differential scattering cross section is the ratio of the scattered intensity to the normal incident irradiance,

$$
\sigma_{d s}=\frac{\partial \sigma_{s}}{\partial \Omega}
$$

When considering the total power absorbed by a scatterer, the total absorption cross section can be similarly defined. The sum of the total scattering cross section and the total absorption cross section is called the total cross section, or extinction cross section,

$$
\sigma_{t}=\sigma_{s}+\sigma_{a}
$$

After normalized by the total cross section and the solid angle, the differential scattering cross section can be used to define a dimensionless quantity called the phase function,

$$
p\left(\hat{\mathbf{s}}_{i n c}, \hat{\mathbf{s}}_{t r}\right)=\frac{4 \pi}{\sigma_{t}} \frac{\partial \sigma_{s}}{\partial \Omega}
$$

where $\hat{\mathbf{s}}_{i n c}$ and $\hat{\mathbf{s}}_{t r}$ are the unit vectors parallel to the incident wave and transmitted wave propagation directions respectively. The phase function represents the amount of scattered power and is not related to the phase of the wave. The name is from astronomy, where it refers to lunar phase. When the absorption is negligible, the phase function integrates to unit over the whole sphere. In many cases, it is the function of just the azimuth angle $\theta$ between $\hat{\mathbf{s}}_{i n c}$ and $\hat{\mathbf{s}}_{t r}$. 
Considering a single scatterer inside the wave propagation, we can study the scattered wave with the Maxwell wave function [68]. When the scatterer size is much smaller than the wavelength, the theory of Rayleigh-Gans scattering is valid. The phase function of a single scatterer under Rayleigh-Gans assumption is proportional to $1+\cos ^{2} \theta$. When the scatterer size is much larger than the wavelength, the scattering is in fact either reflection and refraction or diffraction. The Mie theory describes the scattering by spheres of arbitrary sizes. The phase function of Mie theory is much more involved, and the size of the scatterer is required.

In dealing with media containing many scatterers, we need different theories for different scatterer densities. When the density is tenuous, the incident wave encounters very few scatterers before reaching a receiver. The scattered wave is assumed to be due to a single scattering by a scatterer, and all multiple scatterings are negligible. As the density increases, we need to take into account attenuation due to absorption and scattering along the path. The approximation in this case is that the wave is scattered once by a scatterer, but both the incident wave and the scattered wave are attenuated by absorption and scattering along the path. This includes some of the multiple scattering and is called the first order multiple scattering. Both the single scattering theory and the first order multiple scattering employ the results above.

Historically, there are two methods of investigating multiple scatterings. One is radiative transfer theory, or transport theory, and the other is multiple scattering theory. In analytical theory, or multiple scattering theory, we still start from the Maxwell wave equation including the scattering and absorption characteristics of scatterers. Although mathematically rigorous, it is impossible to solve for a general solution accounting for all the effects. Various theories dealing with different approximations are formed in their own specific ranges [38]. Transport theory, on the other hand, does not use wave equations. It is a phenomenological description of the energy transportation through media. The central equation is the radiative transport equation, or the Boltzmann equation. It is the equation of the radiance [38], or the distribution 
function defined as the radiance divided by the propagation speed [9]. It includes the contribution from the physical processes of absorption, emission, propagation and scattering of radiation. A detailed discussion can be found in refs. $[9,38]$.

When scatterers are densely distributed in media, the diffusion approximation is applicable, and the transport equation is simplified to the diffusion equation [38]. The diffusion approximation assumes the irradiance of scattered wave has the angular dependence of incident wave direction unit vector up to the first order. The phase function is a function of scattered azimuth angle $\theta$ only, and can be expanded in a series of Legendre function. The Heyney-Greenstein phase function is used to approximated the diffuse phase function in many cases. The Heyney-Greenstein phase function is first proposed in astronomy for studying the diffusive radiation in the Milky Way [35]. It has the form

$$
p_{H G}(\theta)=\frac{\gamma}{4 \pi} \frac{1-g^{2}}{\left(1+g^{2}-2 g \cos (\theta)\right)^{\frac{3}{2}}},
$$

where $\gamma$ is the spherical albedo, defined as the ratio of the total scattering cross section to the total absorption cross section, and the parameter $g$ is the asymmetry of the phase function, according to the expression

$$
\gamma g=\langle\cos (\theta)\rangle=\int \mathrm{d} \theta p_{H G}(\theta) \cos (\theta) .
$$

The Heyney-Greenstein phase function is useful in numerical computation for its simplicity.

Both numerical simulations and experimental studies of light scattering inside fluorescent screen are found in many places $[50,26,46,4]$. The result depends on the density and the shape of the scatterers in the screen, and the angular distributions of scattered light in literature are not very far from that of Lambertian objects. However, for the purpose of merit function demonstration in our work, the exact functional form is not critical. The scatterer density in mammography screens is too large to apply the single scattering theory. To the first-order approximation, we 
choose the Lambertian assumption for the angular distribution of scattered light after the screen, which is independent of the output position of the scattered light. The spatial distribution changes with the absorption depths of x-ray photons. We assume the spatial distribution of the scattered light in the form of Gaussian function with the width equal to the absorption depths from the exit surface of the screen. The complete generalized BTDF is therefore the product of the angular distribution and the conditional spatial distribution on the x-ray absorption depth.

\subsection{Optical Simulation}

To simulate the optical point response function $\mathrm{p}_{\mathrm{g}}\left(\mathrm{r}^{\prime}, \mathbf{r} \mid \mathbf{R}, z\right)$, we wrote a ray-tracing routine to facilitate the parallel computing of the SNR on the Linux Beowulf system built in our laboratory. With the samples of $\mathbf{R}, \Delta \mathbf{r}$ and $\hat{\mathbf{s}}$, we can get the displacement $\Delta \mathbf{r}^{\prime}$ by tracing a ray starting at the position $\mathbf{R}+\Delta \mathbf{r}$ and the direction $\hat{\mathbf{s}}$. The vector form of Snell's law in $3 D$ space is [9]

$$
\hat{\mathbf{s}}_{t r}=\frac{n}{n^{\prime}}\left(\hat{\mathbf{s}}_{i n c} \cdot \hat{\mathbf{n}}_{\perp}\right) \hat{\mathbf{n}}_{\perp}+\left[\sqrt{1-\left(\frac{n}{n^{\prime}}\right)^{2}\left(\hat{\mathbf{s}}_{i n c} \cdot \hat{\mathbf{n}}_{\perp}\right)^{2}}\right] \hat{\mathbf{n}},
$$

where $\hat{\mathbf{s}}_{i n c}$ and $\hat{\mathbf{s}}_{t r}$ are the propagation direction vectors of incident and transmitted rays respectively. $\hat{\mathbf{n}}$ is the unit vector normal to the interface, and $\hat{\mathbf{n}}_{\perp}$ is the unit vector normal to $\hat{\mathbf{n}}$ in the plane of incidence. $\hat{\mathbf{n}}_{\perp}$ can be obtained by applying Gram-Schmidt orthogonalization to $\hat{\mathbf{n}}$ and $\hat{\mathbf{s}}_{i n c}$,

$$
\hat{\mathbf{n}}_{\perp}=\frac{\hat{\mathbf{s}}_{i n c}-\left(\hat{\mathbf{s}}_{i n c} \cdot \hat{\mathbf{n}}\right) \hat{\mathbf{n}}}{\sqrt{1-\left(\hat{\mathbf{s}}_{i n c} \cdot \hat{\mathbf{n}}\right)^{2}}} .
$$

The ray hits on the image plane at $\mathbf{R}+\Delta \mathbf{r}+\Delta \mathbf{r}^{\prime}$.

If a ray intercepts the lens housing, the ray will be removed from the final result to simulate a completely absorptive housing. The ray-tracing scheme simulates exactly the complex vignetting of the lens, and realizes the exact function pass $(\mathbf{r}, \mathbf{R} \mid z)$ as well. In the ray-tracing routine, every interface between glass and air is completely 
transparent without reflection for simplicity. The finite reflection of lens elements can be readily added according to Fresnel reflection coefficients. Our simulation neglects this effect because our primary goal is to demonstrate the usage of the objective merit function in lens design.

\subsection{Positive Semi-definite Functions}

To evaluate the performance of the channelized Hotelling observer, we can compute the squared SNR directly. The positive semi-definiteness of the average covariance function ensures the non-negativity of the squared SNR. Taking a look at the covariance function expression again, we find it is important to ensure the positive semi-definiteness of the background covariance function in the numerical computation. Direct Monte Carlo method cannot guarantee the positive definiteness because small deviations in the elements of the matrix usually break it. The positive semidefinite matrix can be decomposed as a product of another matrix with its transpose, which is analogous to taking a square-root of a non-negative number. Similarly, the positive semi-definite function can be written as the autocorrelation of another function. The existence of such a function is explained in Appendix C. This "square-root" function of the covariance function is thus used in the Monte Carlo application to form the "square-root" of the covariance matrix.

The covariance function of the clustered lumpy background $K_{b}\left(\mathbf{R}_{1}, \mathbf{R}_{2}\right)$ is shiftinvariant since the background is stationary. In fact, we can find a real-valued symmetric function $\Phi(\mathbf{R})$ to construct the covariance function in the form

$$
K_{b}\left(\mathbf{R}_{1}-\mathbf{R}_{2}\right)=\iint \mathrm{d}^{2} R \Phi\left(\mathbf{R}_{1}-\mathbf{R}\right) \Phi\left(\mathbf{R}-\mathbf{R}_{2}\right) .
$$

The exact expression of the construction function $\Phi\left(\mathbf{r}^{\prime}\right)$ is derived in Appendix C. With this function, the third term of the covariance matrix of the final image is given 
as

$$
\begin{aligned}
\mathbf{K}_{i j}^{(3)}= & \iint \mathrm{d}^{2} R \iiint \int \mathrm{d}^{2} r_{1}^{\prime} \mathrm{d}^{2} R_{1} \Phi\left(\mathbf{R}_{1}-\mathbf{R}\right) \mathrm{p}_{t o t}\left(\mathbf{r}_{1}^{\prime}, \mathbf{R}_{1}\right) h_{i}\left(\mathbf{r}_{1}^{\prime}\right) \\
& \cdot \iiint \int \mathrm{d}^{2} r_{2}^{\prime} \mathrm{d}^{2} R_{2} \Phi\left(\mathbf{R}_{2}-\mathbf{R}\right) \mathrm{p}_{t o t}\left(\mathbf{r}_{2}^{\prime}, \mathbf{R}_{2}\right) h_{j}\left(\mathbf{r}_{2}^{\prime}\right) .
\end{aligned}
$$

where $\mathbf{K}_{i j}^{(3)}$ is the $i j^{\text {th }}$ element of the third term of the covariance matrix. The set $\left\{\iiint \int \mathrm{d}^{2} r_{1}^{\prime} \mathrm{d}^{2} R_{1} \Phi\left(\mathbf{R}_{1}-\mathbf{R}\right) \mathrm{p}_{t o t}\left(\mathbf{r}_{1}^{\prime}, \mathbf{R}_{1}\right) h_{i}\left(\mathbf{r}_{1}^{\prime}\right)\right\}$ composes a $n^{2} \times 1$ functions vector. The matrix function formed by the product of the vector function and its transpose is thus a positive-definite matrix. The integral of this positive-definite matrix over the field of view is the third term of the image covariance matrix, and therefore positive definite. We will then compute the Monte Carlo integration over the vector function directly. After the above procedure, the resultant estimate of the third term is always positive definite.

\subsection{Channel Profiles}

In chapter 2, statistical decision theory is introduced as the theoretical basis for task-based image quality evaluation. The Bayesian observer is the optimal observer performing classification tasks by this theory, and it is also called the ideal observer. To model human observer's performance in a task-based sense, we should also start from the ideal observer. When modifying the ideal observer to predict the human observer's performance, we should be based on the features of the human visual system.

Vision science is itself a large and active research domain; a review can be found in $[9,30]$. The human has a sophisticated visual perception system from eyes to visual cortex in brain. After an image is formed by the lenses in eyes on the retina, millions of rods and cones are stimulated. The stimuli are then sent to ganglion cells through bipolar cells. The axons of ganglion cells form the optical nerve stream. The stream ends up in the lateral geniculate nucleus of the thalamus. The cells in the lateral 
geniculate nucleus lead the signal into the part of cortex called visual cortex. Some signal processings and feature extractions are performed in the visual cortex layer, so we can perceive the object being viewed.

A model replicating the entire visual system in the human is not necessary to us; instead we need a model that simulates the human data in the kinds of tasks for image quality assessment. In psychophysics, the human's overall response to a pattern is measured to define the human's pattern detectability. In the studies of human's response to the multiple-frequency patterns, scientists come to the conclusion that different spatial-frequency components are detected by independent processors at different narrow ranges of spatial frequencies $[15,58,32]$. These processors are called channels. There is a long history of the channel study in the vision science research. Many cells in the lowest visual areas of cat and monkey and presumably human have separate excitatory and inhibitory regions in their receptive fields. Those cells are called simple cells in the ground-breaking work of Hubel and Wiesel [37]. Very soon after their original studies, the existence of orientation-selective cortex cells was suggested as the bases for existing psychophysical results and inspired new experiments using oriented stimuli. Later investigators have discovered that both simple and cortical cells have another important property. Not only are they selective for the orientation of a line or grating but they are also selective for the width of the line. Gabor functions, one type of windowed sinusoids, are used as stimuli or channels to decompose patterns. This is related to the fact that they are good descriptions of neurons' spatiotemporal weighting functions and that their mathematical properties might have implications for visual processing $[21,70,71]$ Several other kinds of functions have been used instead of Gabor functions. Difference-of-Gaussians (sometimes called DOGs) [24,34] Gaussian-derivative models [71], Laplacian operators [47] and Cauchy functions [42] have all been used in research.

The channelized Hotelling observer matches human observer performance in a variety of signal types and background types together with different noise mechanisms. 
It was first introduced by Myers and Barrett [51] to demonstrate its ability to predict human's performance in correlated noise. Then, the channelized Hotelling observer was found to correlate well with human performance in tomographic imaging system evaluation $[22,16]$, image-compression algorithm evaluation $[1,28,27]$ and the presence of anatomical backgrounds in diagnostic images [20]. The selection of channel sets highly depends on forms of the signals and backgrounds. We use the radially symmetric Difference-Of-Gaussian (DOG) channels because the signal profiles in the image are mostly close to radially symmetric. With the signal known in this task, the channel are centered at the signal location in images. The channel profiles are given in spatial-frequency space with the form

$$
C_{j}(\rho)=\exp \left[-\frac{1}{2}\left(\frac{\rho}{B \sigma_{j}}\right)^{2}\right]-\exp \left[-\frac{1}{2}\left(\frac{\rho}{\sigma_{j}}\right)^{2}\right],
$$

where $\rho$ is the spatial radial frequency variable. The standard deviation of each channel is defined by $\sigma_{j}=\sigma_{0} \alpha^{j}$ from an initial value $\sigma_{0}$. The multiplicative factor $B>1$ defines the bandwidth of the channel. When implementing the channels in the discrete image space, we use the inverse Fourier transform followed by a digitization operator $\mathcal{D}$ defined earlier. The spatial profile of the DOG channel $t_{j}\left(r^{\prime}\right)$ by the inverse Fourier transform of $C_{j}(\rho)$ can be written analytically as

$$
t_{j}(\mathbf{r})=2 \pi\left(B \sigma_{j}\right)^{2} \exp \left[-2 \pi^{2}\left(B \sigma_{j} r\right)^{2}\right]-2 \pi \sigma_{j}^{2} \exp \left[-2 \pi^{2}\left(\sigma_{j} r\right)^{2}\right]
$$

where $r$ is the length of the $2 D$ spatial vector $\mathbf{r}$. To compare the response of different channels, the channel profile is normalized so that $2 \pi \int \mathrm{d} r^{\prime} t_{j}\left(r^{\prime}\right)^{2}=1$,

$$
t_{j, \text { norm }}(\mathbf{r})=\sqrt{\frac{B^{2}+1}{\pi \sigma_{j}^{2}\left(B^{2}-1\right)^{2}}} t_{j}(\mathbf{r}) .
$$

After applying a digitization operator $\mathcal{D}$ on the normalized channel profiles, we can have the vector form of the spatial channel profiles. The channel response on an image is computed by directly applying the vector form of spatial channel profiles onto the image and calculating the inner product of the channel with the image. 


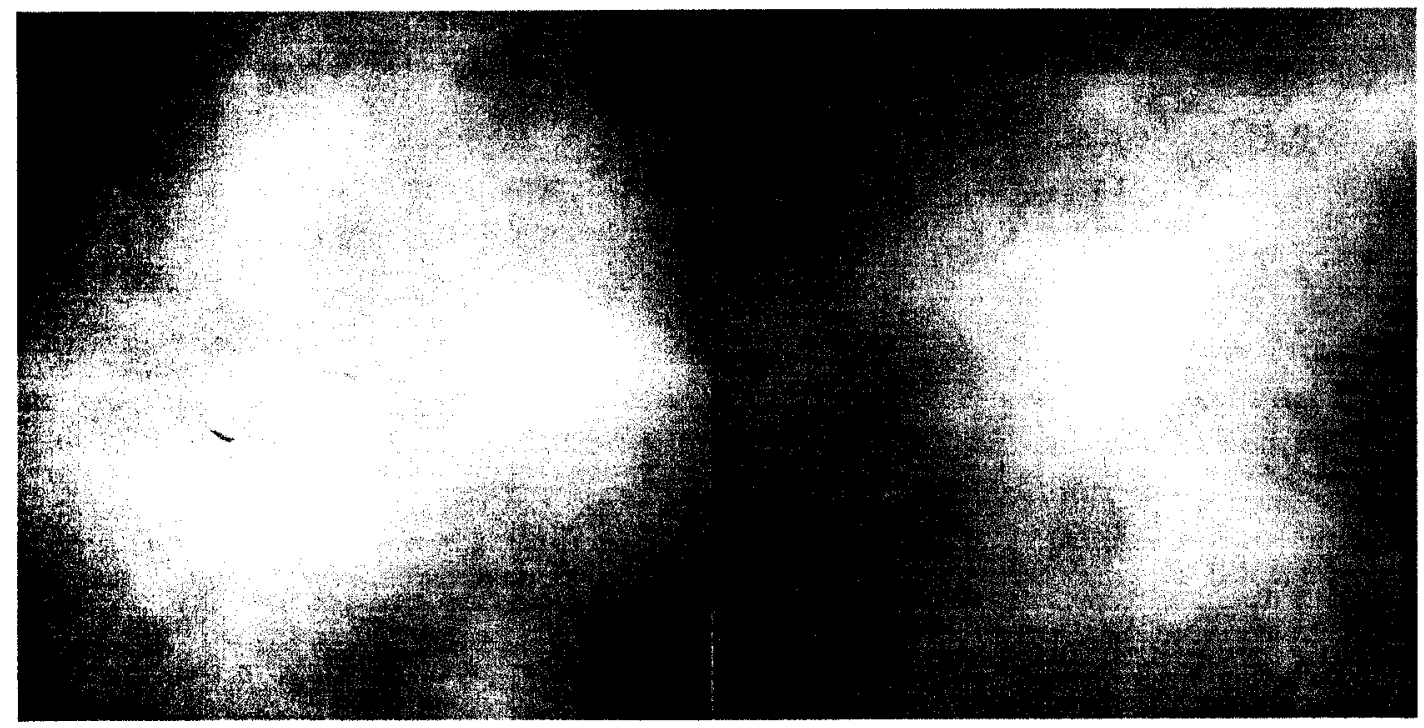

Figure 6.1. Two samples of the clustered lumpy background 


\section{Chapter 7}

\section{Results}

The clustered lumpy background has the same parameters throughout the simulation. The characteristic lengths of each blob before any random rotation are $L_{x}=1.5 \mathrm{~mm}, L_{y}=0.6 \mathrm{~mm}$. The mean number of blobs per cluster is $\bar{K}_{b l o b}=20$ and the mean number of the clusters per square centimeter is $\bar{N}_{\text {cluster }}=10.17 \mathrm{~cm}^{-2}$. The average number of blobs $\bar{K}_{\text {blob }}$ should not be confused with the mean number of secondaries per primary $\bar{K}$ in the previous discussion. The adjustable coefficients in the blob's exponent are $\alpha=3.6373 \mathrm{~mm}^{\frac{1}{2}}, \beta=0.5$, and the standard deviation of the distribution density of the blob centers inside a cluster is $\sigma_{\phi}=3.6 \mathrm{~mm}$. The signal we used is a Gaussian-profile blob with the standard deviation of $\sigma=1.0 \mathrm{~mm}$. The maximum signal level at its center is $10 \%$ of the mean background level. A unit level in the background and signal corresponds to $58.1 \mathrm{x}$-ray photons so that the exposure on the screen is comparable to the typical screen-film mammographic system with anti-scattering grid which is about $9.5 \mathrm{mR}$ [56].

The parameters of the fluorescent screen are largely from the Kodak company's mammographic screens, which use gadolinium oxysulfide activated with terbium as the phosphor. The size of the screen is $24 \times 30 \mathrm{~cm}^{2}$ and the thickness is $84 \mu \mathrm{m}$. The $\mathrm{x}-$ ray absorption coefficient is $\alpha_{x}=1.3 \mathrm{~cm}^{-1}$. On average, each absorbed x-ray photon produces 1000 visible photons which can come out of the exit surface of the screen regardless the absorption depth. It is the value of $\bar{m}$. To get the $Q$ factor in the screen model, the Swank factor $S$ is needed $[63,62,64,13]$. The value of $S$ for this screen is 0.8 . The $Q$ factor is independent of the absorption depth as well and can be found from the equation (4.3.11). To the first order approximation, the generalized BTDF can be written as the product of the separate spatial and angular parts. We 
approximate the spatial distribution density of the visible photons as Gaussian with the standard deviation equal to the absorption depth with respect to the exit surface of the fluorescent screen. The angular part is independent of the positions and depths. The angular distributions are assumed to follow the Lambertian approximation.

The imaging lens is the part containing parameters to be changed. We will study the effects of those parameters on the image quality defined by the SNR of the channelized Hotelling observers. There is a folding mirror between the screen and the lens to eliminate the x-ray photons from striking onto the CCD camera directly. In the simulation, the mirror is unfolded and not included in the ray-tracing routine. The blocked $x$-ray photons are taken away from the simulations.

The CCD camera has $128 \times 128$ pixels with the pixel size $50 \times 50 \mu \mathrm{m}^{2}$. The quantum efficiency is $100 \%$, and there are no gaps between adjacent pixels. This simple model of an ideal CCD camera is adequate for our simulation now.

The channels used in the observer model are the dense DOG channels mentioned in Craig Abbey's work [2]. This model uses ten channels. The initial standard deviation in frequency is $\sigma_{0}=0.005$ pixel $^{-1}$, and the ratio of the standard deviations between adjacent channels is $\alpha=1.4$. The multiplicative factor defining the bandwidth of the channel is $B=1.67$.

The signal position varies from an on-axis spot up to the edge of the screen. The signal position is known to the observer so that the center of the channel set is on the image of the signal center each time. When the magnification is not large enough to cover the full screen in CCD shot, we will move the CCD accordingly to image up to the edge. Each time the CCD camera is shifted, the signal to be imaged will be guaranteed within the CCD detector and not too close to the boundary. 


\subsection{Aperture Size}

The first experiment is to study the effect of the aperture size of the lens. The plots of SNR vs. signal position at different aperture sizes are in Fig. 7.1, and the corresponding spot diagrams at four different fields are in Fig. 7.2. The CCD camera is at the minimum RMS point size position, which is commonly called the best focus position. An iris is used at the stop to control the numerical aperture of the lens. With the parameters unchanged except the iris diameter, the aperture size of the imaging system can be controlled. The CCD camera remains at the minimum RMS spot size position as the aperture size is changed throughout this experiment. When the iris is opened up, the light throughput becomes larger so that the exposure during the same time interval increases. The relatively large demagnification of the lens cuts off a large portion of the light by the relatively small numerical aperture in object space. The visible light that finally gets onto the CCD detector is so dim that the lens usually becomes the quantum sink. Larger stop size generally improves the discrimination ability of observers by making the lens less of a quantum sink. Meanwhile, more aberrations are introduced into the image and make the images more blurred. The signal is gradually smeared into the background by more and more blurs. Blurring effects are usually detrimental to the observer's ability to pick a signal out of the background. The trade-off between the stop size and the blur, i.e. between the numerical aperture and the aberration, can be defined quantitatively by a certain aperture size which makes the observer have the highest SNR.

In this experiment, we vary the object-space numerical aperture from 0.005 up to 0.055 , which corresponds to f-numbers from 10 down to 0.91 . The SNR curves vs. the signal position are compared in all f-number cases. From the Fig. 7.1, the SNR increases with the decreasing f-number at all fields in the f-number range from 10 to 2 . The enhancement in the SNR demonstrates that the flux increase improves the observer performance at the relatively small apertures. From f-number 2 to 
under 1, the SNR has only limited increase at first and then is maximized at about f/1. Although we cannot open the aperture further, we can already observe the saturation of the SNR at around $\mathrm{f} / 1$. In this case, it will not help in detecting signals by decreasing the f-number further. The maximum SNR curve numerically defines the best f-number for this lens design. This f-number quantitatively indicates the balancing point between the flux and the aberrations in the task-based manner.

At the same f-number, the aberration changes with changing field although the exposure is almost the same within a $10^{\circ}$ field of view in this Lambertian condition. Therefore the aberration differences are the main reason for the SNR differences at the different signal positions given any single f-number. From the largest f-number at 10 down to 2, each SNR curve is almost flat though. From the spot diagram plots, the spot size is limited in about 2 by 2 pixels throughout the full range of the field of view. Therefore, the observer performance is insensitive to the aberration changes when the spot width is within about 2 pixels. From $\mathrm{f}$-number 2 to under 1 , the SNR is not flat but has a maximum at about 0.7 field. From the spot diagrams, the spot size generally increases with the decreasing f-number and has the minimum at about 0.7 field. Most of the spots are wider than 2 pixels. This means the observer performance is sensitive to the aberration when the spot size is beyond the 2-pixel limit. Looking at the 0.7 field in the spot diagrams, we find that the spot sizes are still around 2 pixels at different f-number. The SNR increase trend with decreasing f-numbers is again observable at that field. Away from the 0.7 field, both aberration and exposure have visible effects on the observer performance. Even though the spot size is less in the far off-axis field than in the near-axis field, the SNR is higher in the near-axis field than that far from axis. This is because vignetting begins to reduce the exposure in the off-axis field at low f-number, and the SNR enhancement from the smaller spot size is overcompensated by the SNR decrease from less exposure. 


\subsection{Working Distance}

The second experiment is to study the effect of the working distance change of the same lens. The SNR plots at different working distances are in Fig. 7.3, and the corresponding spot diagrams are in Fig. 7.4. We choose the f/0.91 lens design from the previous experiment since it yields best observer performance. Each time we change the working distance, the lens is adjusted to the best focus position for that working distance. The closer the object is to the lens, the farther the CCD camera is from the lens, and the higher the absolute magnification value. The magnification of the imaging system varies monotonically with the working distance, so we use the magnification to denote the working distance. At the designed working distance, the magnification is -0.10 . This experiment examined the SNR changes at the different magnifications from -0.07 to -0.13 . Because of the large demagnification used, the change in the working distance is much smaller than the distance between the screen and the lens. The observer's performance varies considerably in spite of the small changes in the working distance.

The flux becomes larger when increasing the absolute magnification because the solid angle viewed from the object plane becomes larger with the increased working distance. At the close-to-axis field range, the SNR curves decrease with the increasing absolute magnifications. In this field range, the spot size quickly increases when the absolute magnification gets larger. The SNR drop shows that the small improvement on the observer performance by the flux increase cannot overcome the negative effects of blurring. At lower absolute magnification portion, the curves are largely overlapped with an insignificant decreasing trend with increasing absolute magnifications. From the spot diagrams, the spot sizes are within the 2-pixel width limit in this portion. Therefore the observer performs similarly since the flux difference is small. When extending to the far off-axis field region, the changing trend of the SNR curves versus the magnifications reverts, SNR becoming higher at the higher absolute 
magnification. For magnifications lower than 0.1, SNR increases quickly when the absolute magnification gets larger. For the cases when the absolute magnifications are higher than 0.1 , the SNR curves are very close. The spot size now has a minimum at the magnification -0.10 and increases toward both ends. Therefore the rapid improvement in the observer performance below the magnification -0.1 indicates the help from both the decreasing spot size and the increasing flux. When the absolute magnification is larger than 0.1 , the detrimental effects from the increased spot size balance the positive effects from the flux, and the observer performance remains the same. At about 0.7 field, the spot sizes vary little among different working distances, which makes the SNR almost unchanged.

When the absolute magnification is small, the SNR curves are relatively flat at the close-to-axis field range, and generally go downward at the far off-axis field region. This is because the observer can tolerate the aberrations within 2-pixel wide spots, which is true in the near-axis field. At the higher absolute magnification area, the effects from the aberrations become visible, and the observer performs best at about 0.7 field since the spot sizes are minimum there. Although the spot sizes are obviously different between the full field and zero field, the observer performs similarly. This indicates the effects of aberration should be more than just the effects of the spot size.

In this experiment, both the flux and the aberration clearly affects the observer performance. The spot size is only a coarse summary on the effect of the aberrations. More detailed effects by the aberration should not be overlooked. We can select the working distance based on the region where the signal will show up. Above all, there is only small improvement in the near-axis fields and larger drop in the far off-axis fields at the small absolute magnifications, and nearly no improvement in all fields at large absolute magnifications. We should consider the original designed working distance as the best among all choices. 


\subsection{Defocus}

The last experiment is on the focus shift of the lens. The SNR plots at different image plane positions are in Fig. 7.5, and the corresponding spot diagrams are in Fig. 7.6. Based on the first two experiments, we would like to study the effect of the aberration alone. We designed the third experiment since the defocus is the most convenient way to control the blur size of the imaging lens. Additionally, we can check if the best focal plane defined by the smallest RMS spot size is the best in task-based sense. In this experiment, we use the $\mathrm{f} / 0.91$ lens from the first experiment again. The defocus is in terms of CCD detector plane shift from the best focal plane. The shift ranges from $-200 \mu \mathrm{m}$ to $400 \mu \mathrm{m}$, where the negative shift means moving the CCD toward the lens and the positive shift moving away. Now the fluxes in all the cases are the same. At the near-axis field region, The SNR becomes smaller when the CCD camera is moved closer to the lens. When the focus shift is between $100 \mu \mathrm{m}$ and $300 \mu \mathrm{m}$, the SNR stays close and almost always remains at the maximum. When going out to the far off-axis field region, the SNR increases when the CCD camera is moved closer to the lens. When the shift is positive, the SNR curves overlap and remain at the upper bound. The complicated changes in SNR are related to the complex blurs by the different amount of aberrations. From the spot diagrams, the SNR change can approximately explained by the RMS spot size, but we can also see the effect from the further details of aberrations. For the spots at the far off-axis field region and the negative focus shift, the RMS spot size increases when moving closer to the lens while the SNR is almost the same. This indicates that the tail in the spot does not affect the observer performance, even though it contributes to the RMS spot size. A similar situation happens in the near-axis field region and the moderate positive focus shift; the tail around a much "darker" center affects the SNR little. Based on these observations, the RMS spot size is not a very pertinent measure of the position of the best focus. In fact, we can see the best focal plane should be moved a little 
further away from the lens, within $100 \mu \mathrm{m}$ from the RMS best focal plane, as seen from the SNR curve of the $100 \mu \mathrm{m}$ focus shift. 


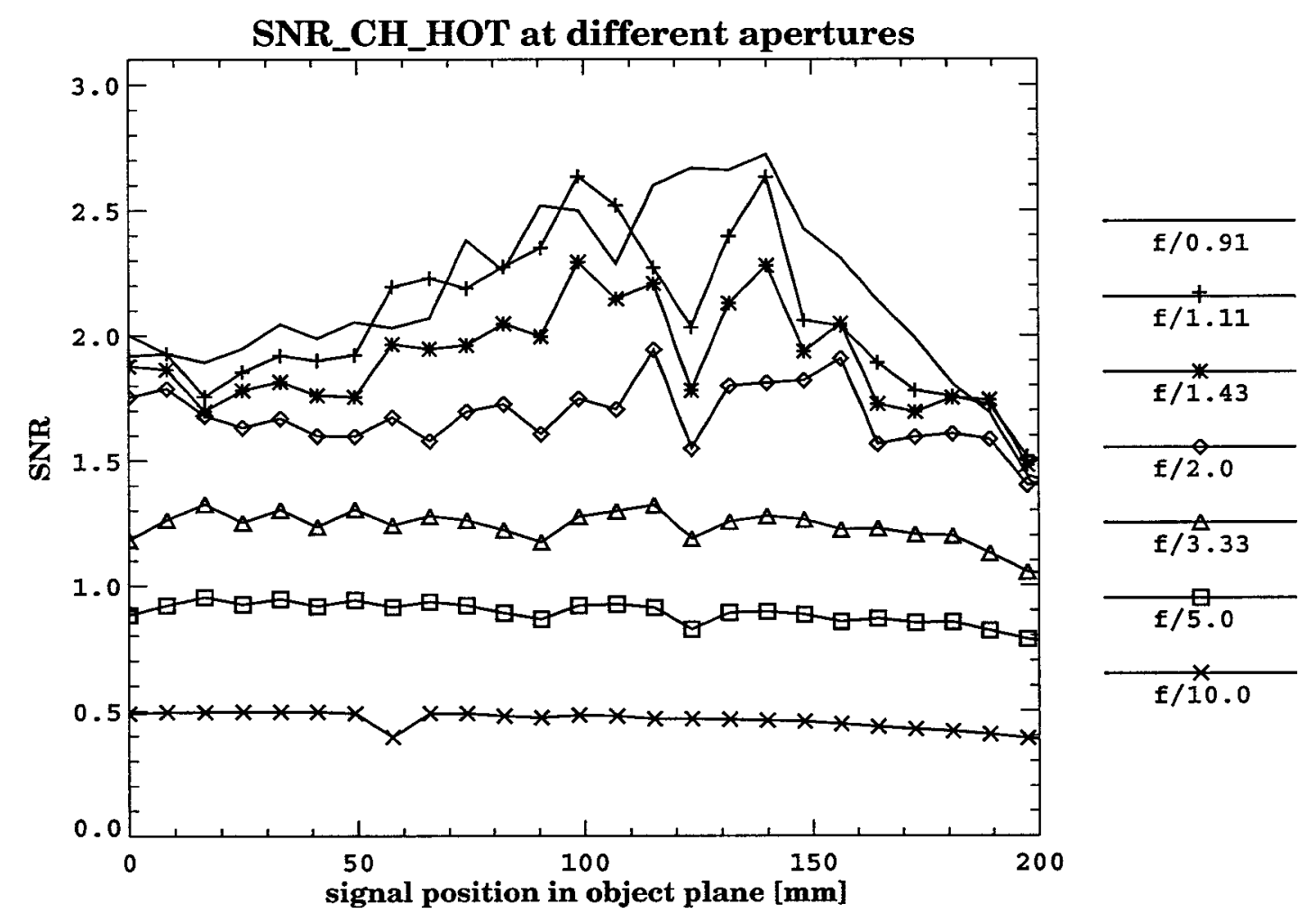

FiguRE 7.1. SNR plots at different aperture sizes. The signal position is measured from the optical axis on the entrance surface of the screen. The image plane position is unchange in all cases. The aperture size is in terms of the numerical aperture in object space. 


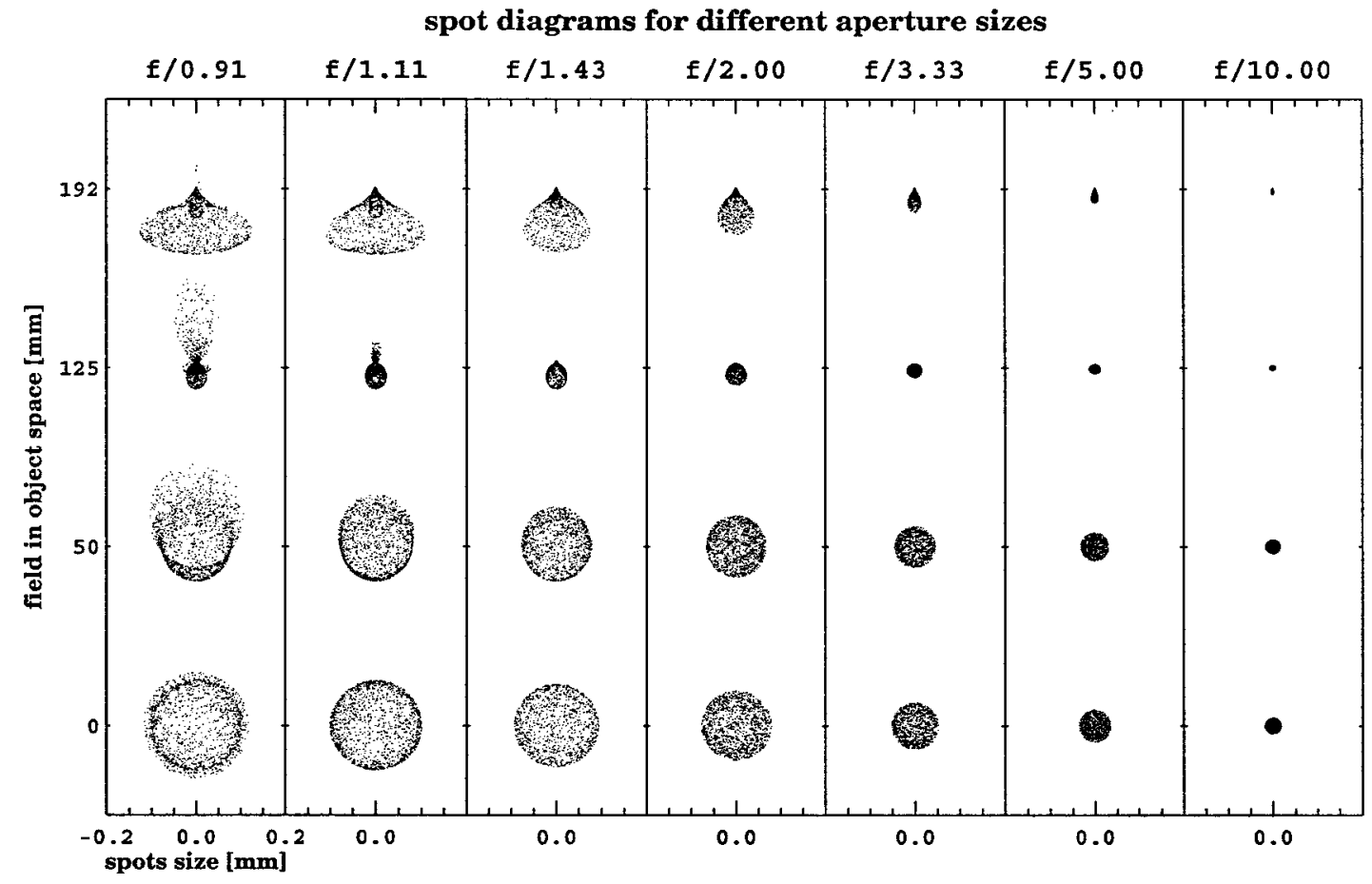

FIguRE 7.2. The spot diagrams at four fields of different aperture sizes. The field position is in terms of the radial distance from the optical axis on the image plane. 


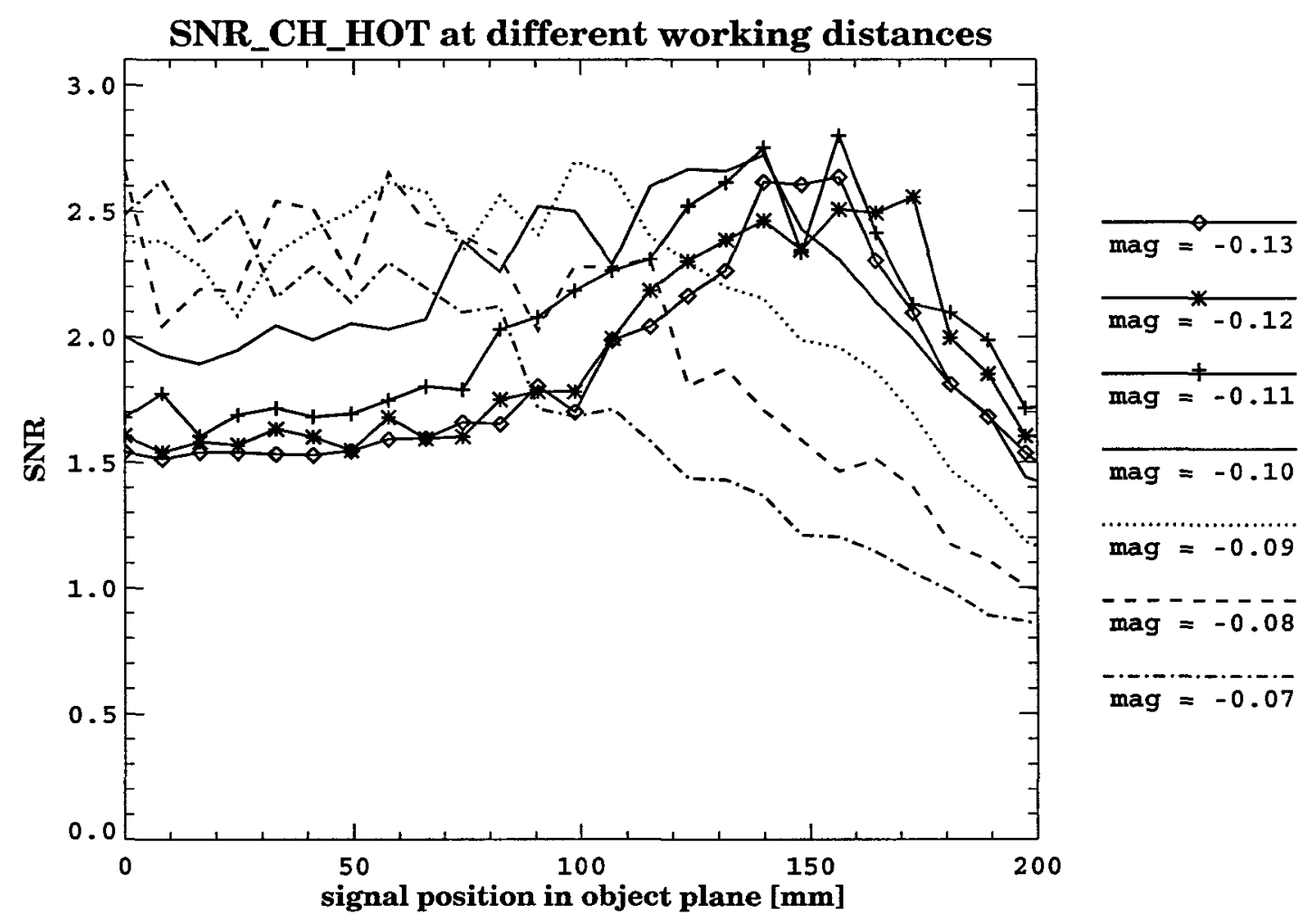

FIgURE 7.3. SNR plots at different working distances. The signal position is measured from the optical axis on the entrance surface of the screen. The aperture size is unchanged in all cases, with NA $=0.055$. The working distance is in terms of the magnification. 
spot diagrams for different magnifications

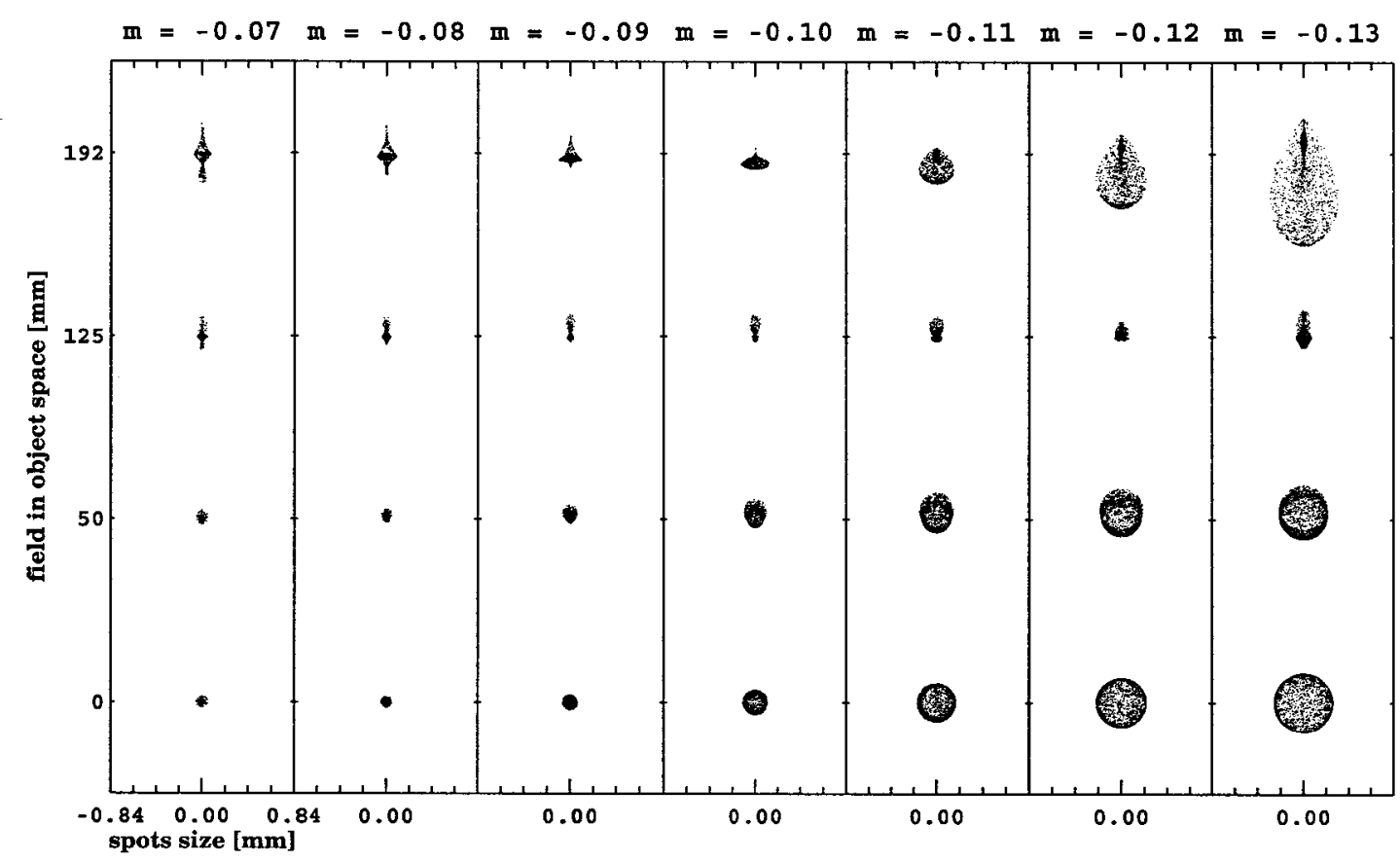

FIgURE 7.4. The spot diagrams at four fields of different working distances. The field position is in terms of the radial distance from the optical axis on the image plane. 


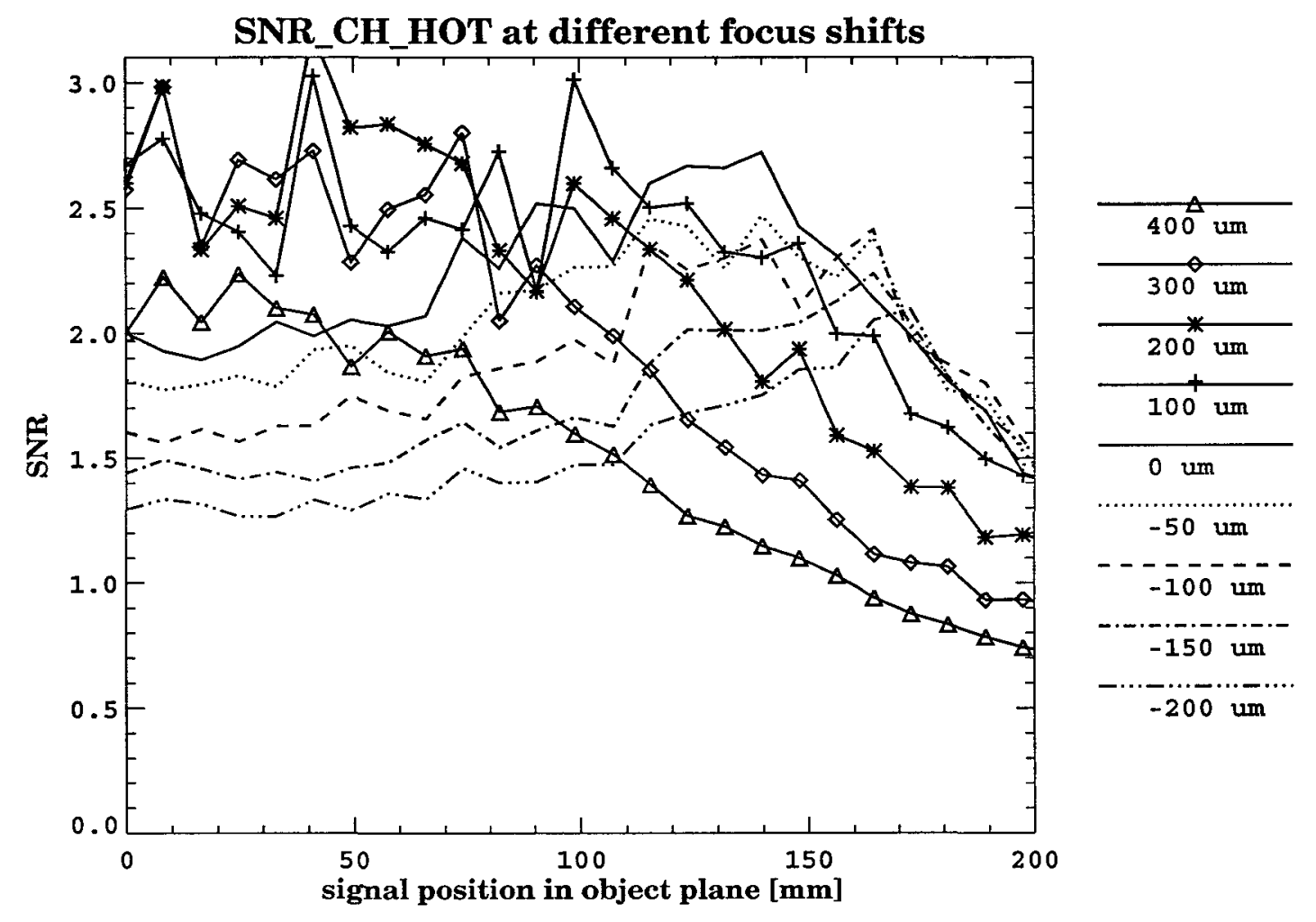

FIgURE 7.5. SNR plots at different image plane positions. The signal position is measured from the optical axis on the entrance surface of the screen. The aperture size is unchange in all cases. The defocus is in terms of the distance from the ideal image plane in image space. 


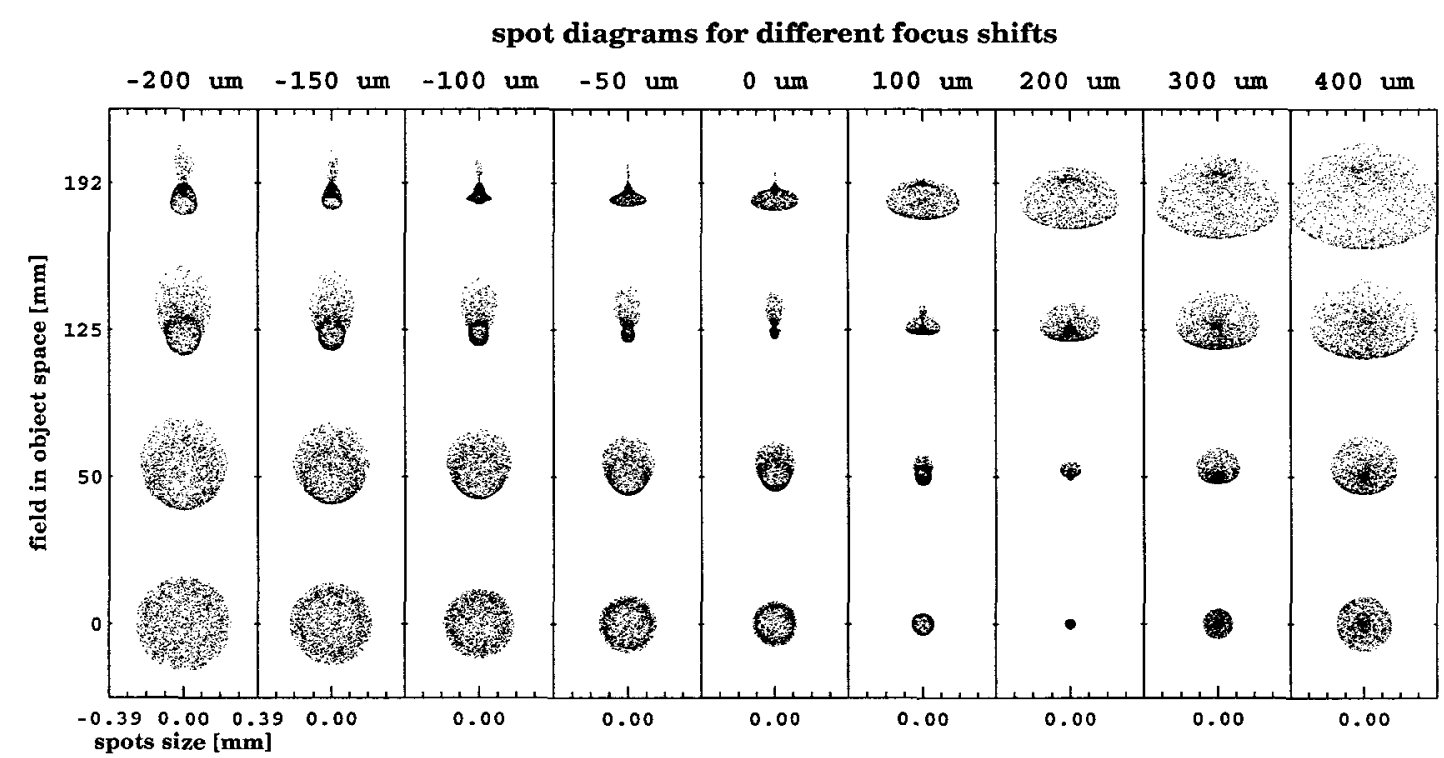

Figure 7.6. The spot diagrams at four fields of different image plane positions. The field position is in terms of the radial distance from the optical axis on the image plane. 
Chapter 8

\section{Conclusions and Future Work}

\subsection{Conclusions}

From our simulation, the task-based lens design merit function in terms of the SNR of the channelized Hotelling observers can quantitatively take into account not only aberrations but also the flux and noises. The designers' subjective opinion on the relationship between the aberration and other facts of the design is eliminated. The connection is instead numerically established based on statistical signal detection theory and the mathematical human vision model through the channelized Hotelling observer. We therefore call the SNR the objective design criterion in task-based sense. To our knowledge, this is the first time to introduce the observer's performance into lens design.

We should note that the results in this work, such as the best f-number at about 1.0 and the observer's tolerance at 2-pixel width spots, are only pertinent to the mammographic imaging condition. To apply the objective criterion, the results are critically determined by the relevant flux level and noise characteristics together with the base lens type.

\subsection{Future Work}

The natural extension of this work is to build an optimization procedure from the quality-evaluation procedure illustrated here. The quality-evaluation step has solved the big problem of numerically computing the task-based merit function. The optimization needs to construct a search algorithm to find the maximum point, preferably the global maximum. 
There are generally two types of search algorithms. One kind of search method is to choose a search direction and take a step in this direction, and then repeat the process iteratively. Most of them need the gradient of the object function under searching, such as iterative coordinate descent, steepest descent and conjugate gradient [9]. Since we already have the analytic formula of the merit function, we can differentiate the function and formulate the expression of the gradient with respect to design parameters. However, the gradient has even more complex form than the merit function itself, and must be more difficult to evaluate as well. It is at least not a good way to use the conventional gradient-involved search algorithm in task-based lens design, if not impractical. Moreover, lens design is a high-dimensional optimization problem, usually involving tens of design parameters. It is common to have multiple local extremes in merit function. The conventional search algorithms can only find a local extreme. The task-based merit function is evaluated by Monte Carlo application, which can introduce additional noise because of the randomness of Monte Carlo applications. This noise may cause errors in conventional search algorithms.

Another more recent type of search algorithms is stochastic search without gradient calculation. These methods, including genetic algorithms, neural networks, simulated annealing and tabu search, are invented to handle complex search problem with high dimension. A brief review can be found in ref. [29]. We propose to investigate these heuristic search algorithms further, especially the simulated annealing algorithm. It is a general optimization algorithm derived from physical science [41]. The algorithm makes analogy to the physical process of annealing, which is a means of reducing the temperature of of a material to its minimum energy state. This state can be considered as a global minimum of the objective function. The negative merit function can be used as the object function in simulated annealing. The simulated annealing process gradually decreases the objective function value by a series of moves in the domain of the function, just like decreasing the temperature slowly to reach the thermal equilibrium. While decreasing the function, some non-improving or value- 
increased steps can be accepted with a probability. This technique simulates the slow temperature-drop, which prevents local suboptimal configurations from being frozen into the material. Mathematically, simulated annealing can find a global minimum instead of a local minimum in this way.

The unique merit function can be readily used in any design optimization as long as the ray-tracing routine is applicable. One step further in this work should be to design a diffractive element for the original designed lens. This piece of optics can correct the original lens to achieve higher observer performance when the lens is used away from its designed imaging conjugate.

The comprehensive analysis of the imaging system developed here can be used in the system study. One of the interesting problems is to investigate non-Gaussian noise in this system when exposures are to small to validate the central limit theorem.

Another consideration would be to introduce proper observer performance measurement for the estimation task, and use it similarly as the merit function for optimizing lenses in the appropriate estimation task. LumiSPECT is a optically coupled SPECT system which employs an imaging optics to detect gamma rays. LumiSPECT is a very similar imaging system as we have studied here, and it should mainly be evaluated for the estimation task. The calculation of the merit function is under study in our group, and great progress has been achieved which makes it ready for imaging system optimization. 


\section{APPENDIX A}

\section{Mean Image Vector}

The discrete mean image array $\overline{\mathrm{g}}$ is given by applying the operator $\mathcal{D}$ on the continuous mean image function $\left\langle g\left(\mathbf{r}^{\prime}\right)\right\rangle$,

$$
\overline{\mathrm{g}}=\left\langle\iint_{\text {supp }} \mathrm{d}^{2} r^{\prime} y_{g}\left(\mathbf{r}^{\prime}\right) \mathbf{h}\left(\mathbf{r}^{\prime}\right)\right\rangle=\iint_{\text {supp }} \mathrm{d}^{2} r^{\prime}\left\langle g\left(\mathbf{r}^{\prime}\right)\right\rangle \mathbf{h}\left(\mathbf{r}^{\prime}\right)=\mathcal{D}\left(\left\langle g\left(\mathbf{r}^{\prime}\right)\right\rangle\right),
$$

where $\mathbf{h}\left(\mathbf{r}^{\prime}\right)$ is the vector function whose elements are the pixel response functions $\left\{h_{i}\left(\mathbf{r}^{\prime}\right)\right\}$.

The calculation of $\left\langle g\left(\mathbf{r}^{\prime}\right)\right\rangle$ is

1. Average over the displacement on the CCD detector plane $\left\{\Delta \mathbf{r}_{n k}^{\prime}\right\}$ :

$$
\begin{aligned}
\left\langle g\left(\mathbf{r}^{\prime}\right)\right\rangle_{\left\{\Delta \mathbf{r}_{n k}^{\prime}\right\}}= & \sum_{n} \sum_{k} \iint_{\infty} \mathrm{d}^{2} r_{n k}^{\prime} \beta_{n k} \delta\left(\mathbf{r}^{\prime}-\mathbf{R}_{n}-\Delta \mathbf{r}_{n k}-\Delta \mathbf{r}_{n k}^{\prime}\right) \\
& \cdot \mathrm{p}_{\mathrm{g}}\left(\mathbf{R}_{n}+\Delta \mathbf{r}_{n k}+\Delta \mathbf{r}_{n k}^{\prime}, \mathbf{R}_{n}+\Delta \mathbf{r}_{n k} \mid \mathbf{R}_{n}, z_{n}\right) \\
= & \sum_{n} \sum_{k} \beta_{n k} \mathrm{p}_{\mathrm{g}}\left(\mathbf{r}^{\prime}, \mathbf{R}_{n}+\Delta \mathbf{r}_{n k} \mid \mathbf{R}_{n}, z_{n}\right) .
\end{aligned}
$$

2. Average over $\left\{\beta_{n k}\right\}$ :

$$
\begin{aligned}
\left\langle g\left(\mathbf{r}^{\prime}\right)\right\rangle_{\left\{\beta_{n k}\right\}} & =\sum_{n} \sum_{k}\left\langle\beta_{n k}\right\rangle \mathrm{p}_{\mathrm{g}}\left(\mathbf{r}^{\prime}, \mathbf{R}_{n}+\Delta \mathbf{r}_{n k} \mid \mathbf{R}_{n}, z_{n}\right) \\
& =\sum_{n} \sum_{k} \operatorname{pass}\left(\mathbf{R}_{n}+\Delta \mathbf{r}_{n k}, \mathbf{R}_{n} \mid z_{n}\right) \mathrm{p}_{\mathrm{g}}\left(\mathbf{r}^{\prime}, \mathbf{R}_{n}+\Delta \mathbf{r}_{n k} \mid \mathbf{R}_{n}, z_{n}\right) .
\end{aligned}
$$

3. Average over the displacements on the exit surface of the screen $\left\{\Delta \mathbf{r}_{n k}\right\}$ :

$$
\begin{aligned}
& \left\langle g\left(\mathbf{r}^{\prime}\right)\right\rangle_{\left\{\Delta \mathbf{r}_{n k}\right\}} \\
= & \sum_{n} \sum_{k} \iint_{\infty} \mathrm{d}^{2} r_{n k} \operatorname{pass}\left(\mathbf{R}_{n}+\Delta \mathbf{r}_{n k}, \mathbf{R}_{n} \mid z_{n}\right) \mathrm{p}_{\mathrm{g}}\left(\mathbf{r}^{\prime}, \mathbf{R}_{n}+\Delta \mathbf{r}_{n k} \mid \mathbf{R}_{n}, z_{n}\right) \\
& \cdot \overline{K_{n}}\left(\mathbf{R}_{n}, z_{n}\right)^{-1} \mathrm{p}_{\mathrm{d}}\left(\mathbf{R}_{n}+\Delta \mathbf{r}_{n k}, \mathbf{R}_{n} \mid z_{n}\right)
\end{aligned}
$$




$$
\begin{aligned}
= & \sum_{n} \overline{K_{n}}\left(\mathbf{R}_{n}, z_{n}\right)^{-1} \sum_{k} \iint_{\infty} \mathrm{d}^{2} r \mathrm{p}_{\mathbf{d}}\left(\mathbf{r}, \mathbf{R}_{n} \mid z_{n}\right) \operatorname{pass}\left(\mathbf{r}, \mathbf{R}_{n} \mid z_{n}\right) \mathrm{p}_{\mathrm{g}}\left(\mathbf{r}^{\prime}, \mathbf{r} \mid \mathbf{R}_{n}, z_{n}\right) \\
= & \sum_{n} \overline{K_{n}}\left(\mathbf{R}_{n}, z_{n}\right)^{-1} K_{n}\left(\mathbf{R}_{n}, z_{n}\right) \\
& \cdot \iint_{\infty} \mathrm{d}^{2} r \mathrm{p}_{\mathrm{d}}\left(\mathbf{r}, \mathbf{R}_{n} \mid z_{n}\right) \operatorname{pass}\left(\mathbf{r}, \mathbf{R}_{n} \mid z_{n}\right) \mathrm{p}_{\mathrm{g}}\left(\mathbf{r}^{\prime}, \mathbf{r} \mid \mathbf{R}_{n}, z_{n}\right)
\end{aligned}
$$

4. Average over the gains $\left\{K_{n}\right\}$ :

$$
\left\langle g\left(\mathbf{r}^{\prime}\right)\right\rangle_{\left\{K_{n}\right\}}=\sum_{n} \iint_{\infty} \mathrm{d}^{2} r \operatorname{p}_{\mathrm{d}}\left(\mathbf{r}, \mathbf{R}_{n} \mid z_{n}\right) \operatorname{pass}\left(\mathbf{r}, \mathbf{R}_{n} \mid z_{n}\right) \mathrm{p}_{\mathrm{g}}\left(\mathbf{r}^{\prime}, \mathbf{r} \mid \mathbf{R}_{n}, z_{n}\right) .
$$

5. Average over the absorption depth $\left\{z_{n}\right\}$

$$
\begin{aligned}
\left\langle g\left(\mathbf{r}^{\prime}\right)\right\rangle_{\left\{z_{n}\right\}}= & \sum_{n} \int_{0}^{d} \mathrm{~d} z_{n} \frac{1}{d_{x}} \cdot \frac{e^{-z_{n} / d_{x}}}{1-e^{-d / d_{x}}} \\
& \cdot \iint_{\infty} \mathrm{d}^{2} r \mathrm{p}_{\mathrm{d}}\left(\mathbf{r}, \mathbf{R}_{n} \mid z_{n}\right) \operatorname{pass}\left(\mathbf{r}, \mathbf{R}_{n} \mid z_{n}\right) \mathrm{p}_{\mathrm{g}}\left(\mathbf{r}^{\prime}, \mathbf{r} \mid \mathbf{R}_{n}, z_{n}\right) .
\end{aligned}
$$

6. Average over the absorption positions of x-ray photons $\left\{\mathbf{R}_{n}\right\}$ :

$$
\begin{aligned}
& \left\langle g\left(\mathbf{r}^{\prime}\right)\right\rangle_{\left\{\mathbf{R}_{n}\right\}} \\
= & \sum_{n} \iint_{\text {supp }} \mathrm{d}^{2} R_{n} \frac{f\left(\mathbf{R}_{n}\right)}{\iint f(\mathbf{R}) \mathrm{d}^{2} R} \int_{0}^{d} \mathrm{~d} z_{n} \frac{1}{d_{x}} \cdot \frac{e^{-z_{n} / d_{x}}}{1-e^{-d / d_{x}}} \\
& \cdot \iint_{\infty} \mathrm{d}^{2} r \mathrm{p}_{\mathrm{d}}\left(\mathbf{r}, \mathbf{R}_{n} \mid z_{n}\right) \operatorname{pass}\left(\mathbf{r}, \mathbf{R}_{n} \mid z_{n}\right) \mathrm{p}_{\mathrm{g}}\left(\mathbf{r}^{\prime}, \mathbf{r} \mid \mathbf{R}_{n}, z_{n}\right) \\
= & \frac{1}{d_{x} \bar{N}(f)} \sum_{n} \iint_{\text {supp }} \mathrm{d}^{2} R_{n} f\left(\mathbf{R}_{n}\right) \int_{0}^{d} \mathrm{~d} z_{n} e^{-z_{n} / d_{x}} \\
& \cdot \iint_{\infty} \mathrm{d}^{2} r \mathrm{p}_{\mathrm{d}}\left(\mathbf{r}, \mathbf{R}_{n} \mid z_{n}\right) \operatorname{pass}\left(\mathbf{r}, \mathbf{R}_{n} \mid z_{n}\right) \mathrm{p}_{\mathbf{g}}\left(\mathbf{r}^{\prime}, \mathbf{r} \mid \mathbf{R}, z_{n}\right) \\
= & \frac{N(f)}{d_{x} \bar{N}(f)} \int_{\text {supp }} \mathrm{d}^{2} R f(\mathbf{R}) \int_{0}^{d} \mathrm{~d} z e^{-z / d_{x}} \\
& \cdot \iint_{\infty} \mathrm{d}^{2} r \mathrm{p}_{\mathrm{d}}(\mathbf{r}, \mathbf{R} \mid z) \operatorname{pass}(\mathbf{r}, \mathbf{R} \mid z) \mathrm{pg}_{\mathrm{g}}\left(\mathbf{r}^{\prime}, \mathbf{r} \mid \mathbf{R}, z\right) .
\end{aligned}
$$


7. Average over the number of absorbed x-ray photons $N$ :

$$
\begin{aligned}
& \left\langle g\left(\mathbf{r}^{\prime}\right)\right\rangle_{N} \\
= & \iint_{\text {supp }} \mathrm{d}^{2} R f(\mathbf{R}) \int_{0}^{d} \mathrm{~d} z \frac{1}{d_{x}} e^{-z / d_{x}} \iint_{\infty} \mathrm{d}^{2} r \mathrm{p}_{\mathrm{d}}(\mathbf{r}, \mathbf{R} \mid z) \operatorname{pass}(\mathbf{r}, \mathbf{R} \mid z) \mathrm{p}_{\mathrm{g}}\left(\mathbf{r}^{\prime}, \mathbf{r} \mid \mathbf{R}, z\right) .
\end{aligned}
$$

8. Average over the x-ray fluence before the entrance surface of the screen $f$ :

$$
\begin{aligned}
& \left\langle g\left(\mathbf{r}^{\prime}\right)\right\rangle_{N} \\
= & \frac{1}{d_{x}} \iint_{\text {supp }} \mathrm{d}^{2} R \bar{f}(\mathbf{R}) \int_{0}^{d} \mathrm{~d} z e^{-z / d_{x}} \iint_{\infty} \mathrm{d}^{2} r \operatorname{p}_{d}\left(\mathbf{r}, \mathbf{R}_{n} \mid z\right) \operatorname{pass}\left(\mathbf{r}, \mathbf{R}_{n} \mid z\right) \mathrm{p}_{\mathbf{g}}\left(\mathbf{r}^{\prime}, \mathbf{r} \mid \mathbf{R}, z\right) .
\end{aligned}
$$

We define a conditional total point response function $\mathrm{p}_{t o t}\left(\mathrm{r}^{\prime}, \mathrm{R} \mid z\right)$, an overall total point response function $\mathrm{p}_{t o t}\left(\mathrm{r}^{\prime}, \mathbf{R}\right)$ and a linear operator $\mathcal{H}_{1}$ now.

$$
\begin{aligned}
\mathrm{p}_{t o t}\left(\mathbf{r}^{\prime}, \mathbf{R} \mid z\right) & =\iint_{\infty} \mathrm{d}^{2} r \mathrm{p}_{\mathrm{d}}(\mathbf{r}, \mathbf{R} \mid z) \operatorname{pass}(\mathbf{r}, \mathbf{R} \mid z) \mathrm{p}_{\mathrm{g}}\left(\mathbf{r}^{\prime}, \mathbf{r} \mid \mathbf{R}, z\right) \\
\mathrm{p}_{\text {tot }}\left(\mathbf{r}^{\prime}, \mathbf{R}\right) & =\frac{1}{d_{x}} \int_{0}^{d} \mathrm{~d} z e^{-d / d_{x}} \mathrm{p}_{t o t}\left(\mathbf{r}^{\prime}, \mathbf{R}\right) \\
{\left[\mathcal{H}_{1} \bar{f}\right]\left(\mathbf{r}^{\prime}\right) } & =\iint_{\text {supp }} \mathrm{d}^{2} R \bar{f}(\mathbf{R}) \mathrm{p}_{t o t}\left(\mathbf{r}^{\prime}, \mathbf{R}\right)
\end{aligned}
$$

The mean image array is

$$
\overline{\mathrm{g}}=\left[\mathcal{D} \mathcal{H}_{1} \bar{f}\right]
$$




\section{APPENDIX B}

\section{COVARIANCE MATRIX}

The covariance matrix $K_{\mathrm{g}}$ can be deduced from the autocorrelation matrix $\mathbf{R}_{\mathrm{g}}$ and the mean image vector $\overline{\mathbf{g}}$.

$$
\mathbf{K}_{\mathbf{g}}=\mathbf{R}_{\mathbf{g}}-\langle\mathbf{g}\rangle\langle\mathbf{g}\rangle^{t}
$$

With the help of the operator $\mathcal{D}$, we can also get the autocorrelation matrix $\mathbf{R}_{\mathbf{g}}$ out of the autocorrelation function $R_{g}\left(\mathbf{r}_{1}^{\prime}, \mathbf{r}_{2}^{\prime}\right)$ :

$$
\begin{aligned}
\mathbf{R}_{\mathbf{g}} & =\left\langle\mathbf{g g}^{t}\right\rangle \\
& =\left\langle\iint \mathrm{d}^{2} r_{1}^{\prime} y_{g}\left(\mathbf{r}_{1}^{\prime}\right) \mathbf{h}\left(\mathbf{r}_{1}^{\prime}\right) \iint \mathrm{d}^{2} r_{2}^{\prime} y_{g}\left(\mathbf{r}_{2}^{\prime}\right) \mathbf{h}^{t}\left(\mathbf{r}_{2}^{\prime}\right)\right\rangle \\
& =\iiint \int \mathrm{d}^{2} r_{1}^{\prime} \mathrm{d}^{2} r_{2}^{\prime} \mathbf{h}\left(\mathbf{r}_{1}^{\prime}\right) \mathbf{h}^{t}\left(\mathbf{r}_{2}^{\prime}\right)\left\langle y_{g}\left(\mathbf{r}_{1}^{\prime}\right) y_{g}\left(\mathbf{r}_{2}^{\prime}\right)\right\rangle \\
& =\left[\mathcal{D} R_{g}\left(\mathbf{r}_{1}^{\prime}, \mathbf{r}_{2}^{\prime}\right) \mathcal{D}^{\dagger}\right] .
\end{aligned}
$$

The exact expression for the autocorrelation function is

$$
\begin{aligned}
R_{g}\left(\mathbf{r}_{1}^{\prime}, \mathbf{r}_{2}^{\prime}\right)= & \left\langle\sum_{n_{1}} \sum_{k_{1}} \beta_{n_{1} k_{1}} \delta\left(\mathbf{r}_{1}^{\prime}-\mathbf{R}_{n_{1}}-\Delta \mathbf{r}_{n_{1} k_{1}}-\Delta \mathbf{r}_{n_{1} k_{1}}^{\prime}\right)\right. \\
& \left.\cdot \sum_{n_{2}} \sum_{k_{2}} \beta_{n_{2} k_{2}} \delta\left(\mathbf{r}_{2}^{\prime}-\mathbf{R}_{n_{2}}-\Delta \mathbf{r}_{n_{2} k_{2}}-\Delta \mathbf{r}_{n_{2} k_{2}}^{\prime}\right)\right\rangle .
\end{aligned}
$$

In the double sum over $n_{1}$ and $n_{2}$, there are $N$ terms with $n_{1}=n_{2}$ and $N^{2}-N$ terms with $n_{1} \neq n_{2}$. For $n_{1}=n_{2}$, there are also $K_{n}$ terms with $k_{1}=k_{2}$ and $K_{n}^{2}-K_{n}$ terms with $k_{1} \neq k_{2}$. For $n_{1} \neq n_{2}$, it is irrelevant whether $k_{1}=k_{2}$. So, there are three cases to consider.

Case 1: $n_{1}=n_{2}$ and $k_{1}=k_{2}$

The calculation parallels the derivation of the mean image array. The contribution from this part is

$$
\left[R_{g}\left(\mathbf{r}_{1}^{\prime}, \mathbf{r}_{2}^{\prime}\right)\right]_{1}=\left[\mathcal{H}_{1} b\right]\left(\mathbf{r}_{2}^{\prime}\right) \delta\left(\mathbf{r}_{1}^{\prime}-\mathbf{r}_{2}^{\prime}\right)
$$


Case 2: $n_{1}=n_{2}$ and $k_{1} \neq k_{2}$

In this case, $\Delta \mathbf{r}_{n k_{1}}^{\prime}$ and $\Delta \mathbf{r}_{n k_{2}}^{\prime}$ are independent, as are $\Delta \mathbf{r}_{n k_{1}}$ and $\Delta \mathbf{r}_{n k_{2}}$. The result from step (3) is

$$
\left[R_{g}\left(\mathbf{r}_{1}^{\prime}, \mathbf{r}_{2}^{\prime}\right)\right]_{2}=\sum_{n} \frac{K_{n}^{2}-K_{n}}{\left\langle K_{n}\right\rangle^{2}} \mathrm{p}_{t o t}\left(\mathbf{r}_{1}^{\prime}, \mathbf{R}_{n} \mid z_{n}\right) \mathrm{p}_{t o t}\left(\mathbf{r}_{2}^{\prime}, \mathbf{R}_{n} \mid z_{n}\right)
$$

Averaging over $K_{n}$ in the next step needs $\left\langle K_{n}\left(\mathbf{R}_{n}, z_{n}\right)\right\rangle$ and $\left\langle K_{n}^{2}\left(\mathbf{R}_{n}, z_{n}\right)\right\rangle$. Although we don't know the PDF of $K_{n}$, we still can go a little further because $K_{n}$ is from the binomial selection of $m\left(\mathbf{R}_{n}, z_{n}\right)$, which is the total number of visible photons generated by the $n^{\text {th }} \mathrm{x}$-ray photon. The probability of a visible photon to come out of the screen is denoted with $A\left(\mathbf{R}_{n}, z_{n}\right)$.

$$
\begin{aligned}
\operatorname{Pr}\left(K_{n}\right) & =\left(\begin{array}{c}
m \\
K_{n}
\end{array}\right) A^{K_{n}}(1-A)^{m-K_{n}}, \\
\overline{K_{n}} & =\bar{m} A \\
\left\langle K_{n}^{2} \mid m\right\rangle & =m A(1-A)+m^{2} A^{2}, \\
\left\langle K_{n}^{2}\right\rangle & =\bar{m} A(1-A)+\overline{m^{2}} A^{2}, \\
\mathrm{Q}\left(\mathbf{R}_{n}, z_{n}\right) & \equiv \frac{\overline{K_{n}^{2}}-\overline{K_{n}}}{\bar{K}_{n}^{2}}, \\
& =\frac{\bar{m} A(1-A)+\overline{m^{2}} A^{2}-\bar{m} A}{\bar{m}^{2} A^{2}}, \\
& =\frac{\overline{m^{2}}-\bar{m}}{\bar{m}^{2}} .
\end{aligned}
$$

With the function $Q\left(\mathbf{R}_{n}, z_{n}\right)$ introduced above, the result from step (4) is

$$
\begin{aligned}
{\left[R_{g}\left(\mathbf{r}_{1}^{\prime}, \mathbf{r}_{2}^{\prime}\right)\right]_{2} } & =\sum_{n} \frac{\overline{K_{n}^{2}}-\overline{K_{n}}}{{\overline{K_{n}}}^{2}} \mathrm{p}_{t o t}\left(\mathbf{r}_{1}^{\prime}, \mathbf{R}_{n} \mid z_{n}\right) \mathrm{p}_{t o t}\left(\mathbf{r}_{2}^{\prime}, \mathbf{R}_{n} \mid z_{n}\right) \\
& =\sum_{n} \mathrm{Q}\left(\mathbf{R}_{n}, z_{n}\right) \mathrm{p}_{t o t}\left(\mathbf{r}_{1}^{\prime}, \mathbf{R}_{n} \mid z_{n}\right) \mathrm{p}_{t o t}\left(\mathbf{r}_{2}^{\prime}, \mathbf{R}_{n} \mid z_{n}\right) .
\end{aligned}
$$

The remaining steps now give

$$
\left[R_{g}\left(\mathbf{r}_{1}^{\prime}, \mathbf{r}_{2}^{\prime}\right)\right]_{2}=\iint_{\text {supp }} \mathrm{d}^{2} R \bar{f}(\mathbf{R}) \int_{0}^{d} \mathrm{~d} z \frac{1}{d_{x}} e^{-z / d_{x}} \mathrm{Q}(\mathbf{R}, z) \mathrm{p}_{t o t}\left(\mathbf{r}_{1}^{\prime}, \mathbf{R} \mid z\right) \mathrm{p}_{t o t}\left(\mathbf{r}_{2}^{\prime}, \mathbf{R} \mid z\right) .
$$


We can express this term more compactly by defining a linear operator $\mathcal{H}_{2}$ :

$$
\left[\mathcal{H}_{2} \bar{f}\right]\left(\mathbf{r}_{1}^{\prime}, \mathbf{r}_{2}^{\prime}\right)=\iint_{\text {supp }} \mathrm{d}^{2} R \bar{f}(\mathbf{R}) \int_{0}^{d} \mathrm{~d} z \frac{1}{d_{x}} e^{-z / d_{x}} \mathrm{Q}(\mathbf{R}, z) \mathrm{p}_{t o t}\left(\mathbf{r}_{1}^{\prime}, \mathbf{R} \mid z\right) \mathrm{p}_{t o t}\left(\mathbf{r}_{2}^{\prime}, \mathbf{R} \mid z\right) .
$$

This part of the autocorrelation function is therefore expressed as

$$
\left[R_{g}\left(\mathbf{r}_{1}^{\prime}, \mathbf{r}_{2}^{\prime}\right)\right]_{2}=\left[\mathcal{H}_{2} \bar{f}\right]\left(\mathbf{r}_{1}^{\prime}, \mathbf{r}_{2}^{\prime}\right)
$$

Case 3: $n_{1} \neq n_{2}$

If $n_{1} \neq n_{2}$, and considering the independence among $\Delta \mathbf{r}_{n_{1}, k_{1}}$ and $\Delta \mathbf{r}_{n_{2}, k_{2}}$, there is no correlation between $\mathbf{R}_{n_{1}}$ and $\mathbf{R}_{n_{2}}$ except possible randomness induced by $f(\mathbf{R})$. For any fixed $f$, step (6) gives

$$
\left[R_{g}\left(\mathbf{r}_{1}^{\prime}, \mathbf{r}_{2}^{\prime}\right)\right]_{3}=\frac{N(f)^{2}-N(f)}{\bar{N}(f)^{2}}\left[\mathcal{H}_{1} f\right]\left(\mathbf{r}_{1}^{\prime}\right)\left[\mathcal{H}_{1} f\right]\left(\mathbf{r}_{2}^{\prime}\right)
$$

Since $N$ is a Poisson random variable at the fixed fluence $f$, we know that

$$
\left\langle N^{2}-N \mid b\right\rangle=\operatorname{Var}(N \mid f)+\bar{N}(f)^{2}-\bar{N}(f)=\bar{N}(f)^{2} .
$$

From the following step, (7), we have

$$
\left[R_{g}\left(\mathbf{r}_{1}^{\prime}, \mathbf{r}_{2}^{\prime}\right)\right]_{3}=\left[\mathcal{H}_{1} f\right]\left(\mathbf{r}_{1}^{\prime}\right)\left[\mathcal{H}_{1} f\right]\left(\mathbf{r}_{2}^{\prime}\right)
$$

After the last step, this case gives

$$
\left[R_{g}\left(\mathbf{r}_{1}^{\prime}, \mathbf{r}_{2}^{\prime}\right)\right]_{3}=\left[\mathcal{H}_{1} R_{f} \mathcal{H}_{1}^{\dagger}\right]\left(\mathbf{r}_{1}^{\prime}, \mathbf{r}_{2}^{\prime}\right)
$$

By adding the three parts (B.0.4), (B.0.12) and (B.0.18) together, we find that the autocorrelation function is

$$
R_{g}\left(\mathbf{r}_{1}^{\prime}, \mathbf{r}_{2}^{\prime}\right)=\left[\mathcal{H}_{1} \bar{f}\right]\left(\mathbf{r}_{2}^{\prime}\right) \delta\left(\mathbf{r}_{1}^{\prime}-\mathbf{r}_{2}^{\prime}\right)+\left[\mathcal{H}_{2} \bar{f}\right]\left(\mathbf{r}_{1}^{\prime}, \mathbf{r}_{2}^{\prime}\right)+\left[\mathcal{H}_{1} R_{f} \mathcal{H}_{1}^{\dagger}\right]\left(\mathbf{r}_{1}^{\prime}, \mathbf{r}_{2}^{\prime}\right)
$$

Because the operator $\mathcal{H}_{1}$ is linear, the covariance function is given as

$$
\begin{aligned}
K_{g}\left(\mathbf{r}_{1}^{\prime}, \mathbf{r}_{2}^{\prime}\right) & =R_{g}\left(\mathbf{r}_{1}^{\prime}, \mathbf{r}_{2}^{\prime}\right)-\left\langle y_{g}\left(\mathbf{r}_{1}^{\prime}\right)\right\rangle\left\langle y_{g}\left(\mathbf{r}_{2}^{\prime}\right)\right\rangle \\
& =\left[\mathcal{H}_{1} \bar{f}\right]\left(\mathbf{r}_{2}^{\prime}\right) \delta\left(\mathbf{r}_{1}^{\prime}-\mathbf{r}_{2}^{\prime}\right)+\left[\mathcal{H}_{2} \bar{f}\right]\left(\mathbf{r}_{1}^{\prime}, \mathbf{r}_{2}^{\prime}\right)+\left[\mathcal{H}_{1} K_{f} \mathcal{H}_{1}^{\dagger}\right]\left(\mathbf{r}_{1}^{\prime}, \mathbf{r}_{2}^{\prime}\right)
\end{aligned}
$$




\section{APPENDIX C \\ Covariance Function Decomposition}

To each positive-definite function $K(x, y)$, there is a Hilbert space $\mathfrak{H}$ with a scalar product $(f, g)$. The positive-definite function has the reproducing property $[9,11]$,

$$
f(y)=(f(x), K(x, y))
$$

The function is therefore also the reproducing kernel of the space $\mathfrak{H}$. In fact, the two concepts of the functions, as the positive definite function and as the reproducing kernel, are known to be equivalent since a reproducing kernel always possesses the characteristic property of positive definite functions [3]. A reproducing kernel can be constructed by a resolution of the identity in a Hilbert space $\mathfrak{H}$ (for a complete study on the resolution of the identity, especially in connection with the theory of operators, see M. H. Stone [61]). We call a resolution of identity a family of projections $\left\{P_{\lambda}\right\}$ in $\mathfrak{H}$, depending on a real parameter $\lambda,-\infty<\lambda<+\infty$, and having some special properties. There is a one-to-one correspondence between the class of all self-adjoint transformations and the class of all resolutions of the identity [61]. A real number $\lambda_{0}$ belongs to the spectrum of $\left\{P_{\lambda}\right\}$ if $P_{\lambda^{\prime \prime}}-P_{\lambda^{\prime}} \neq 0$ for every interval $\lambda^{\prime \prime}-\lambda^{\prime}$ containing $\lambda_{0}$. The numbers belonging to the spectrum form a closed set [3]. It has been proved (theorem of Hellinger-Hahn) that for any spectrum there exists finite or infinite systems $\left\{f_{n}(\lambda)\right\}$ of elements $f_{n}(\lambda) \in \mathfrak{H}$, depending on $\lambda$, such that if we denote by $\Delta f_{n}$ the difference $f_{n}\left(\lambda^{\prime \prime}\right)-f_{n}\left(\lambda^{\prime}\right)$, we have

1. for $m \neq n,\left(\Delta_{1} f_{m}, \Delta_{2} f_{n}\right)=0$ for any intervals $\Delta_{1}, \Delta_{2}$.

2. $\left(\Delta_{1} f_{n}, \Delta_{2} f_{n}\right)=0$ for any non-overlapping intervals $\Delta_{1}, \Delta_{2}$.

3. The elements $\Delta f_{n}$ for $n=1,2, \ldots$ and for all intervals $\Delta$ form a complete system in $\mathfrak{H}$. 
For any system $\left\{f_{n}(\lambda)\right\}$ above, it is seen that $\left\|f_{n}(\lambda)\right\|^{2}$ is a non-decreasing function $\mu_{n}(\lambda)$. We consider the measure $\mu_{n}$, introduced on the real axis by $\mu_{n}(\lambda)$, which leads to Lebesgue-Stieltjes integral $\int \Phi(\lambda) d \mu_{n}(\lambda)[3]$. It has been proved that there exists the limit

$$
\Phi_{n}(x, \lambda)=\lim _{\lambda^{\prime} / \lambda, \lambda^{\prime \prime} \backslash \lambda} \frac{\overline{f_{n}\left(x, \lambda^{\prime \prime}\right)}-\overline{f_{n}\left(x, \lambda^{\prime}\right)}}{\mu_{n}\left(\lambda^{\prime \prime}\right)-\mu_{n}\left(\lambda^{\prime}\right)},
$$

for all $\lambda$ with exception of a set of $\mu_{n}$-measure 0 [3]. We then obtain

$$
\begin{aligned}
& K(x, y)=\sum_{n} \int_{-\infty}^{+\infty} \Phi_{n}(x, \lambda) \overline{\Phi_{n}(y, \lambda)} d \mu_{n} \\
& K(x, x)=\sum_{n} \int_{-\infty}^{+\infty}\left|\Phi_{n}(x, \lambda)\right|^{2} d \mu_{n} .
\end{aligned}
$$

The series and the integrals in (C.0.3) are absolutely convergent because of (C.0.4) [3]. This proves the existence of the decomposition of a positive semi-definite function such as a covariance function.

If the stationary covariance function can be decomposed into $K(\mathbf{R})=\iint \mathrm{d}^{2} r f(\mathbf{R}+\mathbf{r}) f(\mathbf{r})^{*}$, the Fourier transform is a positive function defined as $\left[\mathcal{F}_{2} K\right](\rho)=\|F(\rho)\|$, where $\mathcal{F}_{2}$ is the $2 D$ Fourier transform operator and $F(\rho)$ is the Fourier transform of $f(\mathbf{R})$. The amplitude of the Fourier transform of the decomposition function is the square-root of the Fourier transform of the covariance function.

The covariance function is [14]

$$
\begin{aligned}
K\left(\mathbf{R}_{1}-\mathbf{R}_{2}\right) & =\frac{\bar{N} \bar{K}}{A}\left(R_{b}\left(\mathbf{R}_{1}-\mathbf{R}_{2}\right)+\bar{K} R_{s}\left(\mathbf{R}-1-\mathbf{R}_{2}\right)\right) \\
b\left(\mathbf{R}, \mathcal{R}_{\theta}\right) & =b\left(R, \varphi, \mathcal{R}_{\theta}\right) \\
& =\exp \left[-\alpha R^{\beta} \frac{\sqrt{L_{y}^{2} \cos ^{2}(\theta+\varphi)+L_{x}^{2} \sin ^{2}(\theta+\varphi)}}{L_{x} L_{y}}\right]
\end{aligned}
$$


where $b$ is the blob function, $R_{b}$ is the autocorrelation function of the blob averaged over all the orientations,

$$
R_{b}\left(\mathbf{R}_{1}-\mathbf{R}_{2}\right)=\frac{1}{2 \pi} \int_{0}^{2 \pi} \mathrm{d} \theta \iint_{A} \mathrm{~d}^{2} R b\left(\mathbf{R}_{1}-\mathbf{R}, \mathcal{R}_{\theta}\right) b\left(\mathbf{R}_{2}-\mathbf{R}, \mathcal{R}_{\theta}\right),
$$

and $R_{s}$ is the autocorrelation function of $S_{\theta}$ averaged over all the orientations, given by

$$
\begin{aligned}
R_{s} & =\frac{1}{2 \pi} \int_{0}^{2 \pi} \mathrm{d} \theta \iint_{A} \mathrm{~d}^{2} R S_{\theta}\left(\mathbf{R}_{1}-\mathbf{R}, \mathcal{R}_{\theta}\right) S_{\theta}\left(\mathbf{R}_{2}-\mathbf{R}, \mathcal{R}_{\theta}\right), \\
S_{\theta}(\mathbf{R}) & =\iint \mathrm{d}^{2} R^{\prime} \phi\left(\mathbf{R}^{\prime}\right) b\left(\mathbf{R}-\mathbf{R}^{\prime}, \mathcal{R}_{\theta}\right),
\end{aligned}
$$

where $S_{\theta}$ is the convolution of the blob center PDF $\phi$ and the blob function. The blob center PDF is Gaussian with the standard deviation $\sigma_{\phi}$. The constant $\bar{N}$ is the mean number of clusters within the field of view, $\bar{K}$ is the mean number of blobs per cluster and $A$ the the region of the field of view.

The Fourier transform of $R_{b}$ results in the absolute squared Fourier transform of the blob function averaged over all the orientations:

$$
\left[\mathcal{F}_{2} R_{b}\right](\rho)=\frac{1}{2 \pi} \int_{0}^{2 \pi} \mathrm{d} \theta\left\|\left[\mathcal{F}_{2} b\right](\rho, \theta)\right\|^{2}
$$

The Fourier transform of $R_{s}$ is similarly given as

$$
\left[\mathcal{F}_{2} R_{s}\right](\rho)=\frac{1}{2 \pi} \int_{0}^{2 \pi} \mathrm{d} \theta\left\|\left[\mathcal{F}_{2} S_{\theta}\right](\rho, \theta)\right\|^{2}
$$

and the Fourier transform of $S_{\theta}$ is the product of the Fourier transform of the blob function and the Gaussian $\phi$.

$$
\left[\mathcal{F}_{2} S_{\theta}\right](\rho, \theta)=\exp \left(-2 \pi^{2} \sigma_{\phi}^{2} \rho^{2}\right)\left[\mathcal{F}_{2} b\right](\rho, \theta) .
$$

The Fourier transform of the covariance function is given as

$$
\left[\mathcal{F}_{2} K_{b}\right](\rho)=\frac{\bar{N} \bar{K}}{A}\left(1+\bar{K} e^{\left(-4 \pi^{2} \sigma_{\phi}^{2} \rho^{2}\right)}\right) \cdot \frac{1}{2 \pi} \int_{0}^{2 \pi} \mathrm{d} \theta\left\|\mathcal{F}_{2} b(\rho, \theta)\right\|^{2}
$$


The amplitude of the Fourier transform of the decomposition function is the squareroot of that of the covariance function.

$$
|F(\rho)|=\sqrt{\frac{\bar{N} \bar{K}}{A}\left(1+\bar{K} e^{\left(-4 \pi^{2} \sigma_{\phi}^{2} \rho^{2}\right)}\right)} \cdot \sqrt{\left\langle\left\|\mathcal{F}_{2} b(\rho, \theta)\right\|^{2}\right\rangle_{\theta}} .
$$

where $\left\langle\left\|\mathcal{F}_{2} b(\rho, \theta)\right\|^{2}\right\rangle_{\theta}$ is the averaged absolute square of the Fourier transform of the blob function.

The Fourier transform of the blob function can be simplified as follows,

$$
\begin{aligned}
& {\left[\mathcal{F}_{2} b\right](\rho, \theta) } \\
= & {\left[\mathcal{F}_{2} b\right]\left(\rho, \varphi^{\prime}, \theta\right) } \\
= & \int_{0}^{\infty} \int_{0}^{2 \pi} \mathrm{d} r \mathrm{~d} \varphi r b(r, \varphi, \theta) e^{-j 2 \pi \rho r \cos \left(\varphi^{\prime}-\varphi\right)} \\
= & \int_{0}^{\infty} \int_{0}^{2 \pi} \mathrm{d} r \mathrm{~d} \varphi r \exp \left(-\alpha r^{\beta} \frac{\sqrt{L_{y}^{2} \cos ^{2}(\theta+\varphi)+L_{x}^{2} \sin ^{2}(\theta+\varphi)}}{L_{x} L_{y}}\right) e^{-j 2 \pi \rho r \cos \left(\varphi^{\prime}-\varphi\right)} \\
= & \int_{0}^{\infty} \int_{\theta}^{2 \pi+\theta} \mathrm{d} r \mathrm{~d} \varphi r \exp \left(-\alpha r^{\beta} \frac{\sqrt{L_{y}^{2} \cos ^{2}(\varphi)+L_{x}^{2} \sin ^{2}(\varphi)}}{L_{x} L_{y}}\right) e^{-j 2 \pi \rho r \cos \left(\varphi^{\prime}+\theta-\varphi\right)}, \text { (C. } .0 .1
\end{aligned}
$$

Because the spatial function to be transformed and the Fourier transform kernel are both periodic functions of the variable $\varphi$ with $2 \pi$ as one period, the integral over one period is independent of the starting point of the integration interval. The interval in the above expression can be changed to $(0,2 \pi)$.

$$
\begin{aligned}
& {\left[\mathcal{F}_{2} b\right]\left(\rho, \varphi^{\prime}, \theta\right) } \\
= & \int_{0}^{\infty} \int_{0}^{2 \pi} \mathrm{d} r \mathrm{~d} \varphi r \exp \left(-\alpha r^{\beta} \frac{\sqrt{L_{y}^{2} \cos ^{2}(\varphi)+L_{x}^{2} \sin ^{2}(\varphi)}}{L_{x} L_{y}}\right) e^{-j 2 \pi \rho r \cos \left(\varphi^{\prime}+\theta-\varphi\right)} \\
= & {\left[\mathcal{F}_{2} b\right](\rho, \varphi+\theta, 0) }
\end{aligned}
$$

The Fourier transform of the blob function is still a periodic function of $\theta$ with a period of $2 \pi$, so

$$
\begin{aligned}
\left\langle\left\|\mathcal{F}_{2} b(\rho, \theta)\right\|^{2}\right\rangle_{\theta} & =\left\langle\left\|\mathcal{F}_{2} b(\rho, \varphi+\theta, 0)\right\|^{2}\right\rangle_{\theta} \\
& =\left\langle\left\|\mathcal{F}_{2} b(\rho, \theta, 0)\right\|^{2}\right\rangle_{\theta}
\end{aligned}
$$


The absolute square of the transform averaged over all the orientation is a radially symmetric function at last. For a radially symmetric function, the inverse Fourier transform is actually the Hankel transform, and the corresponding spatial function is a $2 D$ real function. Based on this fact, the phase of the Fourier transform of the decomposition function is uniformly 0 . The function can be calculated by the inverse Fourier transform of the amplitude given above,

$$
f(\mathbf{R})=\mathcal{F}_{2}^{-1}\left(\sqrt{\frac{\bar{N} \bar{K}}{A}\left(1+\bar{K} e^{\left(-4 \pi^{2} \sigma_{\phi}^{2} \rho^{2}\right)}\right)} \cdot \sqrt{\left\langle\left\|\mathcal{F}_{2} b(\rho, \theta, 0)\right\|^{2}\right\rangle_{\theta}}\right) .
$$

The Fourier and inverse Fourier transform are done by the FFT algorithm in a computer for this work.

For non-stationary stochastic processes, the Wigner distribution function can be used in decomposing covariance functions. In that way, our method with Fourier transform can be useful. 


\section{APPENDIX D \\ Probability-Generating Function}

For a non-negative integer-valued random variable $n$, we can define the probabilitygenerating function $M_{n}(\rho)$ as

$$
M_{n}(\rho)=\left\langle\rho^{n}\right\rangle_{n}=\sum_{n=0}^{\infty} \rho^{n} \operatorname{Pr}(\rho) .
$$

It can be considered as the $z$-transform of the probability series $\operatorname{Pr}(n)$ when the real-valued $\rho$ is substitued with the complex variable $z$, with $z^{n}$ instead of $z^{-n}$ in the summation. By changing the variable $\rho$ with $e^{i 2 \pi \xi}$, we can get the characteristic function from the probability-generating function. Moments of the random variable $n$ can be derived from the characteristic function through differentiation. Similarly, when evaluating each derivative of the probability-generating function at the origin, we have

$$
\operatorname{Pr}(n)=\left.\frac{1}{n !} \frac{\partial^{n}}{\partial \rho^{n}} M_{n}(\rho)\right|_{\rho=0} .
$$

which implies the name probability-generating function.

The probability-generating function can generate not only probabilities but factorial moments. When the derivatives of the probability-generating function is evaluated at 1 rather than 0 , the factorial moments of the random variable $n$ are obtained:

$$
c_{k}=\langle n(n-1)(n-2) \cdots(n-k+1)\rangle_{n}=\left.\frac{\partial^{k}}{\partial \rho^{k}} M_{n}(\rho)\right|_{\rho=1},
$$

where $c_{k}$ denotes the $k^{\text {th }}$ factorial moment. The moment-generating function is related to the probability-generating function by a simple variable change. The momentgenerating function of the non-negative integer-valued random variable $n$ is defined as

$$
\Phi(t)=\left\langle e^{n t}\right\rangle_{n}=\sum_{n=0}^{\infty} e^{n t} \operatorname{Pr}(n) .
$$


The $k^{t h}$ moment can be computed by the $k^{\text {th }}$ derivative of the moment-generating function evaluated at 0 ,

$$
\left\langle n^{k}\right\rangle_{n}=\left.\frac{\partial^{k}}{\partial t^{k}} \Phi(t)\right|_{t=0}
$$

We should remember that moment-generating function is defined for all kinds of random variables, not just non-negative integer-valued ones. 


\section{REFERENCES}

[1] Craig K. Abbey and Harrison H. Barrett. Linear iterative reconstruction algorithms: study of observer performance. In Yves Bizais, Christian Barillot, and Robert DiPaola, editors, Information Processing in Medical Imaging, number 3 in Computational Imaging and Vision, pages 65-76, Dordrecht, 1995. Kluwer Academic.

[2] Craig K. Abbey and Harrison H. Barrett. Human- and model-observer performance in ramp-spectrum noise: effects of regularization and object variability. Journal of the Optical Society of America A, 18(3):473-488, Mar 2001.

[3] N. Aronszajn. Theory of reproducing kernels. Transactions of the American Mathematical Society, 68(3):337-404, May 1950.

[4] Aldo Badano and Rachel Leimbach. Depth-dependent phosphor blur in indirect x-ray imaging sensors. In Larry E. Antoruk and Martin J. Yaffe, editors, Medical Imaging 2002: Physics of Medical Imaging, volume 4682 of Proceedings of SPIE, pages 94-106. Society of Photo-optical Instrumentation Engineers, SPIE-the International Society for Optical Engineering, May 2002.

[5] Harrison H. Barrett. Objective assessment of image quality: effects of quantum noise and object variability. Journal of Optical Society of America, A, 7(7):12661278, Jul 1990.

[6] Harrison H. Barrett, Craig K. Abbey, and Eric Clarkson. Objective assessment of image quality. iii. roc metrics, ideal observers, and likelihood-generating functions. Journal of Optical Society of America, A, 15(6):1520-1535, Jun 1998.

[7] Harrison H. Barrett, Craig K. Abbey, Brandon Gallas, and Miguel P. Eckstein. Stablized estimates of hotelling-observer detection performance in patientstructured noise. In Harold L. Kundel, editor, Medical Imaging 1998: Image Perception, volume 3340 of Proceedings of SPIE, pages 27-43. Society of Photooptical Instrumentation Engineers, SPIE-the International Society for Optical Engineering, Feb 1998.

[8] Harrison H. Barrett, J. L. Denny, Robert F. Wagner, and Kyle J. Myers. Objective assessment of image quality. ii. fisher information, fourier crosstalks, and figures of merit for task performance. Journal of Optical Society of America, A, 12(5):834-852, May 1995.

[9] Harrison H. Barrett and Kyle J. Myers. Foundations of Image Science. Wiley Series in Pure and Applied Optics. John Wiley and Sons, Inc, 2004. 
[10] Harrison H. Barrett, Robert F. Wagner, and Kyle J. Myers. Correlated point processes in radiological imaging. In Richard L. Van Metter and Jacob Beutel, editors, Physics of Medical Imaging, volume 3032 of Proceedings of SPIE, pages 110-124. Society of Photo-optical Instrumentation Engineers, SPIE-the International Society for Optical Engineering, Feb 1998.

[11] Christian Berg, Jens Peter Reus Christensen, and Paul Russel. Harmonic Analysis on Semigroups: Theory of Positive Definite and Related Functions. Graduate Texts in Mathematics. Springer-Verlag, first edition, 1984.

[12] Jean-Pierre Bissonnette, I. A. Cunningham, David A. Jaffray, A. Fenster, and P. Munro. A quantum accounting and detective quantum efficiency analysis for video portal imaging. Medical Physics, 24(6):815-826, Jun 1997.

[13] I. M. Blevis, D. C. Hunt, and J. A. Rowlands. X-ray imaging using amorphous selenium: detection of swank factor by pulse height spectroscopy. Medical Physics, 25(5):638-641, May 1998.

[14] Francois O. Bochud, Craig K. Abbey, and Miguel P. Eckstein. Statistical texture synthesis of mammographic images with clustered lumpy backgrounds. Optics Express, 4(1):33-43, Jan 1999.

[15] F. W. Campbell and J. G. Robson. Application of fourier analysis to visibility of gratings. Journal of Physiology, 197(3):551-566, Aug 1968.

[16] Ellen B. Cargill. A Mathematical Liver Model and its Application to System Optimization and Texture Analysis. Phd dissertation, University of Arizona, Tucson, Arizona, 1989.

[17] Eric Clarkson, Matthew A. Kupinski, and Harrison H. Barrett. Transformation of characteristic functionals through imaging systems. Optics Express, 10(13):536539, Jul 2002.

[18] I. A. Cunningham, M. S. Westmore, and A. Fenster. A spacial-frequency dependent quantum accounting diagram and detective quantum efficiency model of signal and noise propagation in cascaded imaging systems. Medical Physics, 21(3):417-427, Mar 1994.

[19] Miguel P. Eckstein, Binh Pham, and Criag K. Abbey. The effect of image compression for model and human observers in signal known statistically tasks. In Dev P. Charkraborty and Elizabeth A. Krupinski, editors, Medical Imaging 2002: Image Perception, Observer Performance, and Technology assessment, volume 4686 of Proceedings of SPIE, pages 13-24. Society of Photo-optical Instrumentation Engineers, SPIE-the International Society for Optical Engineering, Apr 2002 . 
[20] Miguel P. Eckstein and J. S. Whiting. Lesion detection in structured noise. Academic Radiology, 2(3):249-253, Mar 1995.

[21] David J. Field. Relations between the statistics of natural images and the response properties of cortical cells. Journal of the Optical Society of America A, 4(12):100-114, Dec 1987.

[22] R. D. Fiete, Harrison H. Barrett, White E. Smith, and Kyle J. Myers. The hotelling trace criterion and its correlation with human observer performance. Journal of the Optical Society of America A, 4(5):945-953, May 1987.

[23] B. Roy Frieden. Probability, Statistical Optics, And Data Testing: A Problem solving Approach. Springer Series in Information Sciences. Springer-Verlag, third edition, 2001.

[24] L. J. Frishman, A. W. Freeman, J. B. Troy, D. E. Schweitzer-Tong, and C. Enroth-Cugell. Spatiotemporal frequency responses of cat retinal ganglion cells. Journal of General Physiology, 89:599-628, Apr 1987.

[25] Keinosuke Fukunaga. Introduction to Statistical Pattern Recognition. Computer Science and Scientific Computing. Morgan Kaufmann, 340 Pine Street, Six Floor, San Francisco, CA 94104-3205, second edition, 1990.

[26] G. E. Giakoumakis and D. M. Miliotis. Light angular distribution of fluorescent screens excited by x-rays. Physics in Medicine and Biology, 30(1):21-29, Jan 1985.

[27] H. C. Gifford, M. A. King, D. J. de Vries, and E. J. Soares. Channelized Hotelling and human observer correlation for lesion detection in hepatic spect imaging. The Journal of Nuclear Medicine, 41(3):514-521, Mar 2000.

[28] H. C. Gifford, R. G. Wells, and M. A. King. A comparison of human observer lroc and numerical observer roc for tumor detection in spect images. IEEE Transactions on Nuclear Science, 46(4):1032-1037, Aug 1999.

[29] Fred Glover and Harvey J. Greenberg. New approaches for heuristic search: a bilateral linkage with artificial intelligence. European Journal of Operational Research, 39:119-130, 1989.

[30] Norma S. Graham. Visual Pattern Analyzers. Oxford Psychology Series. Oxford University Press, first edition, 1989.

[31] Norma S. Graham. Complex channels, early nonlinearities, and normalization in texture segmentation. In Michael S. Landy and J. Anthony Movshon, editors, Computational Models of Visual Processing, chapter 18, pages 273-290. The MIT Press, first edition, 1990. 
[32] Norma S. Graham and J. Nachmias. Detection of grating patterns containing two frequencies: A comparison of single-channel and multiple-channel models. Vision Research, 11(3):251-259, Mar 1971.

[33] K. M. Hanson. Detectability in the presence of computed tomographic reconstruction noise. In Joel E. Gray and William R. Hendee, editors, Application of Optical Instrumentation in Medicine VI, volume 127 of Proceedings of SPIE, pages 304-312. Society of Photo-optical Instrumentation Engineers, American Association of Physicists in Medicine, SPIE-the International Society for Optical Engineering, Jan 1977.

[34] M. J. Hawken and A. J. Parker. Spatial properties of neurons on the monkey striate cortex. Proceedings of the Royal Society of London, Series B, Biological Sciences, 231(1263):251-288, Jul 1987.

[35] L. G. Henyey and J. L. Greenstein. Diffuse radiation in the galaxy. Astrophysical Journal, 93(1):70-83, Jan 1941.

[36] Harold Hotelling. The generalization of student's ratio. Annals of Mathematical Statistics, 2(3):360-378, Aug 1931.

[37] D. H. Hubel and T. N. Wiesel. Ferrier lecture: functional architecture of macaque monkey visual cortex. Proceedings of the Royal Society of London, Series B, Biological Sciences, 198(1130):1-59, May 1977.

[38] Akira Ishimaru. Wave Propagation and Scattering in Random Media. IEEE/OUP Series on Electromagnetic Wave Theory. IEEE Press and Oxford University Press, Oxford University Press, Walton St., Oxford OX2 6DP, 1997.

[39] Philip F. Judy, Richard G. Swensson, and Massimiliano Szulc. Lesion detection and signal-to-noise ratio in ct. Medical Physics, 8(1):13-23, Jan 1981.

[40] Rudolf Kingslake. Lens Design Fundamentals. Academic Press, San Diego, California, 1978.

[41] S. Kirkpatrick, C. D. Jr. Gelatt, and M. P. Vecchi. Optimization by simulated annealing. Science, 220:671-680, 1983.

[42] Stanley A. Klein and Dennis M. Levi. Hyperacuity thresholds of 1 sec: theoretical predictions and empirical validation. Journal of the Optical Society of America A, 2(7):1170-1190, Jul 1985.

[43] Glenn F. Knoll. Radiation Detection and Measurement. John Wiley and Sons, Inc, third edition, 2000. 
[44] Matthew A. Kupinski, Eric Clarkson, John W. Hoppin, Liying Chen, and Harrison H. Barrett. Experimental determination of object statistics from noisy images. Journal of the Optical Society of America A, 20(3):421-429, Mar 2003.

[45] Andrew D. A. Maidment and Martin J. Yaffe. Analysis of the spatial-frequencydependent dqe of optically coupled digital mammography detectors. Medical Physics, 21(6):721-729, Jun 1994.

[46] Andrew D. A. Maidment and Martin J. Yaffe. Analysis of signal propagation in optically coupled detectors for digital mammography: I. phosphor screens. Physics in Medicine and Biology, 40(5):877-889, May 1995.

[47] D. Marr and E. Hildreth. Theory of edge detection. Proceedings of the Royal Society of London, Series B, Biological Sciences, 207(1167):187-217, Feb 1980.

[48] James L. Melsa and David L. Cohn. Decision and Estimation Theory. McGrawHill, Inc, 1221 Avenue of the Americas, New York, NY 1002, first edition, 1978.

[49] Richard Van Metter and Majid Rabbani. An application of multivariate momentgenerating functions to the analysis of signal and noise propagation in radiographic screen-film systems. Medical Physics, 17(1):65-71, Jan 1990.

[50] Daniel J. Mickish. Radiation transfer in medical x-ray intensifying screens. In Roger H. Schneider and Samuel J. Dwyer III, editors, Application of Optical Instrumentation in Medicine XIII, volume 535 of Proceedings of SPIE, pages 148-156. Society of Photo-optical Instrumentation Engineers, American Association of Physicists in Medicine, SPIE-the International Society for Optical Engineering, Feb 1985.

[51] Kyle J. Myers and Harrison H. Barrett. Addition of a channel mechanism to the ideal-observer model. Journal of the Optical Society of America A, 4(12):24472457, Dec 1987.

[52] Kyle J. Myers, Harrison H. Barrett, M. C. Borgstrom, E. B. Cargill, R. D. Fiete, D. D. Patton, G. W. Seeley, W. E. Smith, and M. O. Stempski. A systematic approach to the design of diagnostic systems for nuclear medicine. In S. L. Bach, editor, Information Processing in Medical Imaging: Proceedings of the Ninth Conference, pages 431-444, Martinus Nijhoff, Dordrecht, the Netherland, June 1986. The Clinical Center and The Fogarty International Center of the National Institutes of Health, Kluwer Academic Publishers Group.

[53] M. Rabbani, R. Shaw, and R. Van Metter. Detective quantum efficiency of imaging systems with amplification and scattering mechanism. Journal of the Optical Society of America, A, 4(5):895-901, May 1987. 
[54] Majid Rabbani and Richard Van Metter. Analysis of signal and noise propagation for several imaging mechanisms. Journal of the Optical Society of America, A, 6(8):1156-1165, Aug 1989.

[55] Piotr A. Rodnyi. Physical Processes in Inorganic Scintillators. The CRC Press Laser and Optical Science and Technology Series. CRC Press, 2000 Corporate Blvd., N.W., Boca Raton, Florida 33431, 1997.

[56] Hans Roehrig, Tong Yu, and Elizabeth Krupinski. Image quality control for digital mammographic systems: initial experience and outlook. Journal of Digital Imaging, 8(2):52-66, May 1995.

[57] Jannick P. Rolland and Harrison H. Barrett. Effect of random background inhomogeneity on observer detection performance. Journal of the Optical Society of America A, 9(5):649-658, May 1992.

[58] M. Sachs, J. Nachmias, and J. Robson. Spatial-frequency channels in human vision. Journal of the Optical Society of America, 61(9):1176-1186, Sep 1971.

[59] Bahaa E. A. Saleh and Malvin Carl Teich. Multiplied-poisson noise in pulse, particle, and photon detection. Proceedings of the IEEE, 70(3):229-245, Mar 1982.

[60] Ivar Stakgold. Green's functions and boundary value problems. John Wiley and Sons, Inc, first edition, 1979.

[61] Marshall Harvey Stone. Linear Transformations in Hilbert Space and Their Applications to Analysis, volume 15 of Colloquium Publications (American Mathematical Society). The American Mathematical Society, first edition, 1932.

[62] Robert K. Swank. Absorption and noise in x-ray phosphors. Journal of Applied Physics, 44(9):4199-4203, Sep 1973.

[63] Robert K. Swank. Calculation of modulation transfer function of x-ray fluorescent screens. Applied Optics, 12(8):1865-1870, Aug 1973.

[64] Robert K. Swank. Measurement of absorption and noise in an x-ray image intensifier. Journal of Applied Physics, 45(8):3673-3678, Aug 1974.

[65] Malvin Carl Teich. Role of the doubly stochastic Neyman type-A and Thomas counting distributions in photon detection. Applied Optics, 20(14):2457-2467, Jul 1981. 
[66] David P. Trauernicht and Richard Van Metter. The measurement of conversion noise in x-ray intensifying screens. In Roger H. Schneider and Samuel J. Dwyer, editors, Medical Imaging II: Image Formation, Detection, Processing, And Interpretation, volume 914 of Proceedings of SPIE, pages 100-116. Society of Photo-optical Instrumentation Engineers, SPIE-the International Society for Optical Engineering, Feb 1988.

[67] Harry L. Van Trees. Detection, Estimation, And Modulation Theory Part I. Detection, Estimation, And Linear Modulation Theory. John Wiley and Sons, Inc, first edition, 2001.

[68] H. C. van de Hulst. Light Scattering by Small Particles. Dover Classics of Science and Mathematics. Dover Publications Inc., 180 Varick St., New York, N.Y. 10014, 1981.

[69] Robert F. Wagner. Decision theory and the signal-to-noise ratio of Otto Schade. Photographic Science and Engineering, 22(1):41-46, Jan 1978.

[70] A. B. Watson. Detection and recognition of simple spatial forms. In Oliver John Braddick, Andrew Crofton Sleigh, and Rank Prize Funds, editors, Physical and Biological Processing of Images, volume 11 of Springer Series in Information Sciences, pages 100-114. Springer-Verlag, Sep 1983.

[71] Michael A. Webster and Russell L. De Valois. Relationship between spatialfrequency and orientation tuning of striate-cortex cells. Journal of the Optical Society of America A, 2(7):1124-1132, Jul 1985. 